\title{
Understanding and utilizing waveguide invariant range-frequency striations in ocean acoustic waveguides
}

\author{
by \\ Kevin L. Cockrell \\ B.S., Engineering Physics, University of California, San Diego, 2005 \\ Submitted in partial fulfillment of the requirements for the degree of \\ Doctor of Philosophy \\ at the \\ MASSACHUSETTS INSTITUTE OF TECHNOLOGY \\ and the \\ WOODS HOLE OCEANOGRAPHIC INSTITUTION \\ February 2011 \\ (c)2010 Kevin L. Cockrell. All rights reserved. \\ The author hereby grants to MIT and WHOI permission to reproduce and to distribute \\ publicly paper and electronic copies of this thesis document in whole or in part in any \\ medium now known or hereafter created.
}

Author:

Joint Program in Oceanography/Applied Ocean Science and Engineering Massachusetts Institute of Technology and Woods Hole Oceanographic Institution

September 30, 2010

Certified by:

Henrik Schmidt

Professor of Mechanical and Ocean Engineering

Massachusetts Institute of Technology

Thesis Supervisor

Accepted by:

James C. Preisig

Chair, Joint Committee for Applied Ocean Science and Engineering

Massachusetts Institute of Technology

Woods Hole Oceanographic Institution

Accepted by:

David E. Hardt

Chair, Committee for Graduate Students

Massachusetts Institute of Technology 


\title{
Understanding and utilizing waveguide invariant range-frequency striations in ocean acoustic waveguides
}

by

Kevin L. Cockrell

Submitted to the Joint Program in Applied Ocean Science and Engineering on 30 September 2010, in partial fulfillment of the requirements for the degree of Doctor of Philosophy.

\begin{abstract}
Much of the recent research in ocean acoustics has focused on developing methods to exploit the effects that the sea surface and seafloor have on acoustic propagation. Many of those methods require detailed knowledge of the acoustic properties of the seafloor and the sound speed profile (SSP), which limits their applicability. The range-frequency waveguide invariant describes striations that often appear in plots of acoustic intensity versus range and frequency. These range-frequency striations have properties that depend strongly on the frequency of the acoustic source and on distance between the acoustic source and receiver, but that depend mildly on the SSP and seafloor properties. Because of this dependence, the waveguide invariant can be utilized for applications such as passive and active sonar, time-reversal mirrors, and array processing, even when the SSP or the seafloor properties are not well known. This thesis develops a framework for understanding and calculating the waveguide invariant, and uses that framework to develop signal processing techniques for the waveguide invariant.

A method for passively estimating the range from an acoustic source to a receiver is developed, and tested on experimental data. Heuristics are developed to estimate the minimum source bandwidth and minimum horizontal aperture required for range estimation.

A semi-analytic formula for the waveguide invariant is derived using WKB approximation along with a normal mode description of the acoustic field in a rangeindependent waveguide. This formula is applicable to waveguides with arbitrary SSPs, and reveals precisely how the SSP and the seafloor reflection coefficient affect the value of the waveguide invariant.

Previous research has shown that the waveguide invariant range-frequency striations can be observed using a single hydrophone or a horizontal line array (HLA) of hydrophones. This thesis shows that traditional array processing techniques are sometimes inadequate for the purpose of observing range-frequency striations using a HLA. Array processing techniques designed specifically for observing range-
\end{abstract}


frequency striations are developed and demonstrated.

Finally, a relationship between the waveguide invariant and wavenumber integrations is derived, which may be useful for studying range-frequency striations in elastic environments such as ice-covered waveguides.

Thesis Supervisor: Henrik Schmidt

Title: Professor of Mechanical and Ocean Engineering 


\section{Acknowledgements}

I wish to thank:

My thesis advisor, Professor Henrik Schmidt, for encouraging me to pursue my own ideas while nudging me back on track when I went astray. And let's not forget the trips to NURC. Those were fantastically fun and incredibly educational.

Professor James Preisig for always providing thoughtful responses to my questions in a very timely manner - an uncommon and admirable characteristic in modern academia.

Professor Nick Makris for introducing me to acoustics in an unforgettably elegant manner.

Geoff Fox for going far above and beyond his call of duty, and providing oftenneeded comic relief. Mike Benjamin for his career guidance and keeping LAMSS down-to-earth. Joe Curcio for being such a great travel companion, and providing some outside perspective on the MIT lifestyle. Arjuna for his amazing ability to spread an aura of calm during the most hectic moments at sea, and helping me collect much of the experimental data in this thesis.

My LAMSS labmates Alexis, Maria, and Deep for their friendship and alwayswelcome YouTube distractions. Andrew Shafer for many insightful conversations and Linux help. Costa for always taking the time to answer my random signal processing questions (no pun intended). Raymond for helping with data processing. Toby for operating the AUVs as if he were in The Matrix. Stephanie for reminding LAMSS that we can do oceanography in addition to acoustics. And Andrew Poulsen for his mentorship and friendship.

The capable staff of the NATO Undersea Research Center (NURC) for their collaborations and assistance in collecting much of the data in this thesis.

The United States Office of Naval Research for supporting this thesis research and my entire graduate education. (Specifically: PLUSNet S05-06, GOATS 2005 N00014-05-1-0255, and GOATS 2008 N00014-08-1-0013)

Tim, Chris, Vas, and Jeff for their enduring if long-distance friendships. Phil for introducing me to the sometimes strange ways of MIT, and providing me with many tips on succeeding in academia.

My soon-to-be wife, Debbie Chen, for encouraging me to go to MIT, and for 
her enduring support throughout my time here.

My brothers for their support and for taking care of many family matters during the past five years. My mother for her unconditional support in everything I pursue. And finally my father, for instilling in me an insatiable curiosity for understanding the natural world. 


\section{Contents}

1 Introduction $\quad 13$

1.1 Thesis outline and contributions . . . . . . . . . 15

2 Background 19

2.1 Acoustic propagation in a waveguide . . . . . . . . . . . 19

2.2 Normal modes . . . . . . . . . . . . . . . . . . . 20

2.2.1 Basic interpretation of normal modes . . . . . . . . 21

2.2.2 Types of modes (terminology) . . . . . . . . . 21

2.2 .3 The modal sum . . . . . . . . . . . . . . . 22

2.3 The range-frequency waveguide invariant . . . . . . . . . . . 25

2.3.1 Range-frequency striations . . . . . . . . . . . 25

2.3.2 The waveguide invariant in ideal waveguides . . . . . . 34

2.3.3 The waveguide invariant non-ideal waveguides . . . . . 35

2.4 Chapter summary . . . . . . . . . . . . . . . . 40

3 Robust passive range estimation using the waveguide invariant 43

3.1 Introduction . . . . . . . . . . . . . . 43

3.2 Brief review of range-frequency striations . . . . . . . . . 45

3.3 Using the two-dimensional discrete Fourier transform for range es-

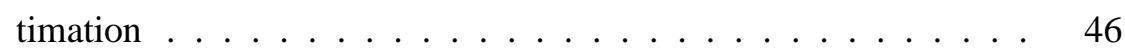

3.3.1 Outline of the 2D-DFT technique . . . . . . . 46

3.3.2 Determining local striation angle with the 2D-DFT . . . 47

3.4 Results . . . . . . . . . . . . . . . . . . . . . . . 59

3.4.1 2D-DFT technique applied to simulated data . . . . 59 
3.4.2 2D-DFT technique applied to experimental data . . . . . 61

3.4.3 Validity of $\beta=1$ assumption . . . . . . . . . 71

3.5 Chapter summary . . . . . . . . . . . . . . 72

4 A WKB modal approach to calculating the waveguide invariant 75

4.1 Introduction . . . . . . . . . . . . . 76

4.2 Calculating $\beta$ using the WKB approximation . . . . . . . 78

4.2.1 Approximating a finite difference with a continuous derivative .................... 83

4.2.2 Implicit differentiation . . . . . . . . . . . 83

4.3 Exploiting dependence on $\omega \ldots \ldots \ldots$. . . . . . . . 86

4.4 Interpretation and implementation . . . . . . . . . . . . . . . 89

4.5 Examples ........................... 91

4.5.1 Iso-velocity SSP: ideal and Pekeris waveguides . . . . . . 92

4.5.2 $n^{2}$-linear waveguide . . . . . . . . . . 95

4.5.3 Quasi-analytic results for a deep-water Munk profile . . . 97

4.5.4 Realistic shallow water waveguide . . . . . . . . . 99

4.6 Calculating $\beta$ for non-adjacent modes . . . . . . . . . . . 101

4.7 Chapter summary . . . . . . . . . . . . . . . . . . 102

4.A Chapter Appendix: Justification for treating $k_{r}$ as an implicit function 105

4.B Chapter Appendix: Derivation of expression for $\beta \ldots \ldots 6$

5 Array processing with the waveguide invariant 109

5.1 Introduction . . . . . . . . . . . . . . . . . . . . . 109

5.2 Review of incoherent processing . . . . . . . . . . . 111

5.3 Coherent processing . . . . . . . . . . . . . . . 111

5.3.1 HLA response to a planewave . . . . . . . . . . 112

5.3.2 HLA response to source in waveguide . . . . . . . . . 114

5.3.3 Array processing for the waveguide invariant . . . . . . 116

5.3 .4 Uniform weighs . . . . . . . . . . . . . 117

5.3.5 Designing array weights for waveguide invariant striations 119

5.4 A simulated example . . . . . . . . . . . . . . . . . 121

5.4 .1 Filter design . . . . . . . . . . . . . 122

5.4 .2 Simulated results . . . . . . . . . . . . . 122 
5.5 Experimental example . . . . . . . . . . . . . . . . . 125

5.6 Chapter summary . . . . . . . . . . . . . . . . . . . . 126

6 A relationship between the waveguide invariant and wavenumber integration 129

6.1 Introduction . . . . . . . . . . . . . . . . . . . . 129

6.2 The waveguide invariant and normal modes in a planar waveguide $\quad 130$

6.3 Relating the waveguide invariant to wavenumber integration . . . 131

6.4 An ideal example . . . . . . . . . . . . . . . . . . . . . . . 134

6.5 Relevance . . . . . . . . . . . . . . . . . . 136

6.6 Chapter summary . . . . . . . . . . . . . . . . . . 136

7 Conclusion 139

7.1 Thesis summary . . . . . . . . . . . . . . . . . . . . . . 140

7.2 Thesis Significance . . . . . . . . . . . . . . . . . . . 141

7.3 Specific suggestions for future research . . . . . . . . . . 141

$\begin{array}{lr}\text { Appendices } & 149\end{array}$

A Review of waveguide invariant literature 149

A.1 General review . . . . . . . . . . . . . . . . . . . . . . . . . . 149

A.2 Rays, modes, and the waveguide invariant . . . . . . . . . 156

B References for the WKB approximation in ocean acoustics 157

C List of symbols and acronyms 


\section{List of Figures}

2.1 Illustration showing the relationship between the sound speed profile, the horizontal wavenumber, and the type of mode. . . . . . . 22

2.2 Graphical representation of the mode summation . . . . . . . 24

2.3 Waveguide invariant striations in a simulated Pekeris waveguide . 28

2.4 Plots illustrating the waveguide invariant for two different simulated shallow-water waveguides . . . . . . . . . . . . 29

2.5 Waveguide invariant striations from experimental data . . . . . . 30

2.6 Waveguide invariant striations for a deep-water waveguide . . . . 31

2.7 Plot of a single cosine term of the acoustic intensity . . . . . . . 33

2.8 Group speed versus phase speed for modes in two shallow-water waveguides .................... 37

2.9 Group speed versus phase speed for modes in a deep-water waveguide 38

3.1 The four steps used to determine the angle of the striations in $I_{\text {win }}(r, f) 52$

$3.2 I_{2 \mathrm{DF}}\left(k_{r, \text { image }}, k_{f \text {,image }}\right)$ showing the full range of $k_{r \text {,image }}$ and $k_{f \text {,image }} 54$

3.3 Acoustic intensity in a Pekeris waveguide plotted versus range and frequency .................. . . 60

3.4 Range estimates for a simulated Pekeris waveguide . . . . . . . 62

3.5 Measured sound speed profiles and bathymetry for passive range estimation experiment . . . . . . . . . . . . 63

3.6 Acoustic intensity versus range and frequency for incoming segment of passive ranging experiment . . . . . . . . 65

3.7 Range estimates for the incoming segment of the experiment . . . 66

3.8 Acoustic intensity versus range and frequency for outgoing segment of passive ranging experiment . . . . . . . 67 
3.9 Range estimates for the outgoing segment of the experiment _ . . 68

3.10 Range estimates for the outgoing segment of the experiment using the array-filtered data . . . . . . . . . . . 70

3.11 Simulated spectrogram for the environment in the passive ranging experiment .................... 72

4.1 Illustration of the WKB approximation applied to calculating the horizontal wavenumbers . . . . . . . . . . . 80

4.2 Plots of $n$ versus $k_{r}$ using the WKB approximation . . . . . . . 81

4.3 Contour plot of $n$ versus $k_{r}$ and $\omega$ for an ideal waveguide . . . . . 82

4.4 The waveguide invariant $\beta$ for an ideal and a Pekeris waveguide, and the functions used to calculate $\beta \ldots \ldots$. . . . . . 94

4.5 The waveguide invariant $\beta$ for an $n^{2}$-linear waveguide, and the functions used to calculate $\beta$ plotted on a common x-axis . . . . 96

4.6 The waveguide invariant $\beta$ for a deep-water Munk SSP, and the functions used to calculate $\beta \ldots \ldots \ldots$. . . . . . 98

4.7 The waveguide invariant $\beta$ for a realistic shallow water waveguide, and the functions used to calculate $\beta \ldots \ldots \ldots$

4.8 The waveguide invariant $\beta$ for non-adjacent modes . . . . . . . 103

5.1 The sound speed profile for the simulated environment . . . . . 123

5.2 Plots of the wavenumber response function of three different array weights, each designed for a different temporal frequency $f \ldots$. . 124

5.3 Filtered versus unfiltered simulated acoustic intensity as a function of range and frequency . . . . . . . . . . . . . . . 125

5.4 Filtered versus unfiltered experimentally measured acoustic intensity as a function of range and frequency . . . . . . . . . . 127

6.1 Wavenumber kernel striations . . . . . . . . . . . . . . 135 


\section{Chapter 1}

\section{Introduction}

Sound provides an unparalleled means of sensing in the vast, dark oceans that cover $70 \%$ of the Earth. Only with sound can one detect a submerged whale from hundreds of miles away [55], or measure the average temperature of an entire ocean in a matter of hours [65]. Although sound is a powerful method for sensing in the ocean, it's an indirect one: the desired information must be extracted from an acoustic signal.

Humans do this type of acoustic sensing on a daily basis (albeit, above the water). A person can determine the type of building he is walking in - a large stone cathedral, or an office building? - based solely on the sound of his footsteps. He could even guess roughly how large the cathedral is, based on how much reverberation he hears. But to answer questions like these quantitatively — how many meters wide is the cathedral? - quantitative methods must be used.

It is usually not obvious how to extract the desired information from the raw acoustic signal, which is nothing more than a time-series of pressure recorded by a hydrophone. The signal usually contains not only the information of interest, but also other information that is not of interest. The sound caused by crashing surface waves may not be of interest when one is trying to detect the presence of whale vocalizations. Conversely, the presence of whale vocalizations may not be of interest when one is trying to detect crashing surface waves. One of the main challenges in ocean-acoustic sensing is to create signal processing techniques that distill out the information of interest (e.g., whale vocalizations) while ignoring 
everything else (e.g., sound of waves crashing).

How does one go about creating such signal processing techniques? Sometimes this can be done with standard engineering techniques, such as using a lowpass filter to reject high-frequency noise. But often in ocean acoustics, the signal processing techniques come from an understanding of the acoustics itself.

For example, ray theory reveals that some of the acoustic energy in the ocean propagates without ever being affected by the seafloor. If one was trying to acoustically determine the sound speed profile (SSP) of the water column, and the properties of the seafloor were unknown and not of interest, one could use ray theory develop a signal processing technique that extracts only the features of the signal that were not affected by the seafloor. The output of such a signal processing technique could then be used to infer properties of the water column without ever having to be concerned with the properties of the seafloor.

This thesis focuses on a feature of acoustic propagation in a waveguide known as the "waveguide invariant." The waveguide invariant is a parameter denoted by $\beta$ which summarizes the frequency dependence of a waveguide's Green's function. The waveguide invariant is important not only because it's a fundamental property of the ocean acoustic waveguide, but also because it can be exploited to extract useful information from an acoustic signal when the details of the ocean environment (the SSP and seafloor properties) are unknown.

Many signal processing technique that utilize the physics of waveguide propagation, such as matched field processing, require detailed knowledge of the ocean environment. The accuracy of such methods tends to be very sensitive to mismatches between the actual environment and the assumed environment. In contrast, signal processing techniques based on the waveguide invariant often require only a minimal amount of knowledge about the environment. Because of this, techniques based on the waveguide invariant are applicable even when the details of the SSP or the seafloor are unknown.

The waveguide invariant has been used for a wide range of applications such as: passive range estimation [58, 56, 62], matched field processing [59, 23], active sonar $[49,27,25]$, array processing $[37,57,66]$, time-reversal mirrors $[32,54,38$, 39], and more (See Appendix A for a complete review of the waveguide invari- 
ant literature). Despite its many uses, the waveguide invariant is not well studied compared to other ocean-acoustic phenomena.

The goal of this thesis is to improve upon the latest understanding of the waveguide invariant and related signal processing so that future applications of the waveguide invariant can be better executed.

\subsection{Thesis outline and contributions}

Chapters 3 - 6 are the original research contributions of this thesis.

Chapter 2 This chapter provides the technical background for the thesis; it explains normal mode propagation in a range-independent waveguide, and provides a detailed introduction to the range-frequency waveguide invariant.

Chapter 3 This chapter presents one of the most straight-forward applications of the waveguide invariant: estimating the range to a broadband acoustic source in a shallow-water waveguide using a single acoustic receiver towed directly toward the acoustic source. Previous research has shown that a twodimensional Fourier transform can be used to extract information about the slope of the waveguide invariant striations. This chapter extends that research by relating the ocean-acoustic environmental parameters to the signal processing parameters, and applying the resulting signal processing technique to estimate the range to the acoustic source (assuming $\beta=1$ ). Heuristics are developed to estimate the minimum source bandwidth and minimum horizontal aperture required for range estimation. The range estimation algorithm is tested on experimental and simulated data for source ranges of $500-2200 \mathrm{~m}$ and frequencies from 350 to $700 \mathrm{~Hz}$. The algorithm is accurate to within approximately $25 \%$ for the cases tested and requires only a minimal amount of a priori knowledge about the environment.

Chapter 4 This chapter addresses the question: "Why is the waveguide invariant, invariant?" Although it has been shown analytically that $\beta \approx 1$ for ideal waveguides, numerical and experimental results have revealed that $\beta \approx 1$ for many (but not all) realistic shallow-water waveguides as well. Ocean- 
acoustic techniques that utilize the waveguide invariant, such as those presented in Ch. 3, often assume $\beta=1$. Therefore, it is important to understand why $\beta \approx 1$ in many realistic waveguides, and when $\beta$ deviates greatly from 1 .

This chapter presents a method for calculating $\beta$ using a modal WKB description of the acoustic field in a range-independent waveguide, which reveals a straightforward relationship between the SSP and $\beta$. That relationship is used to illustrate why non-uniformities in the SSP sometimes have such a small effect on $\beta$ and under what circumstances the non-uniformities will have a large effect on $\beta$. The method relies on implicit differentiation and thus does not explicitly solve for the horizontal wavenumbers of the modes, making it applicable to waveguides with arbitrary sound speed profiles and fluid bottom half-spaces. Several examples are given, including an analytic estimate of $\beta$ in a Pekeris waveguide.

Chapter 5 This chapter generalizes a previously known result which showed that a horizontal line array (HLA) can be used to observe waveguide invariant striations from one source while rejecting noise from other sources. It is shown that array weights commonly used for planewave beamforming, such as uniform weights, can have the unintended effect of suppressing some the desired striations. Insights gained from Ch. 4 are used to show that even when noise is not present, array processing can still be useful because it can suppress components of the striation pattern that are not useful for acoustic sensing. Experimental data is used to illustrate the ability of an array processor to preserve the desired striations while rejecting noise.

Chapter 6 This chapter shows that although the waveguide invariant is typically defined in terms of normal modes or ray theory, it can also be related to the wavenumber-integration method for calculating the acoustic field in a waveguide. The Wiener-Khinchin Theorem is used to show that the autocorrelation of the wavenumber-integration kernel, when plotted versus wavenumber difference and frequency, contains striations that can be described by the waveguide invariant. 
Chapter 7 This chapter summarizes and concludes the thesis. Several specific suggestions for future areas of research are given.

Appendix A This appendix is an extensive but brief review of the waveguide invariant literature, most of which is not required to support the conclusions of the thesis but is included for the interested reader. 


\section{Chapter 2}

\section{Background}

This chapter briefly reviews the aspects of ocean acoustics that are relevant for this thesis - normal modes in a range-independent waveguide — and then provides a detailed introduction to the range-frequency waveguide invariant.

\subsection{Acoustic propagation in a waveguide}

We restrict our attention the acoustic field caused by a point source in a rangeindependent ocean waveguide. As far the acoustics is concerned, the environment is fully described by the sound speed profile (SSP) in the water column, and the sound speed and density profile of the seafloor. If one is only interested in the acoustic field in the water column, then the seafloor can be fully characterized by its reflection coefficient as function of horizontal wavenumber and frequency.

As simple as that physical model may seem, a tremendous amount of research was required to develop techniques to accurately calculate the acoustic field in such a waveguide. The acoustic field be calculated and conceptually understood using ray theory, normal modes, or wavenumber integration. Other methods can be used for calculating the acoustic field, such as finite element analysis or the parabolic equation, but those methods do not lend themselves to understanding the underlying acoustics of propagation in a waveguide.

The concepts in this thesis are most readily understood using the normal mode description of the acoustic field. 


\subsection{Normal modes}

This section explains the aspects of normal modes that are important for understanding the waveguide invariant, including some obscure topics not typically covered in textbooks. A full derivation and interpretation of normal modes can be found in [33, Chs. 2 and 5], as well as [60] and [10].

When the acoustic source and receiver are separated by a distance greater than a few water depths, the complex pressure as a function of range from the source $r$, depth $z$, and frequency $\omega$ can be written as [33, Eq. 5.14]

$$
p(r, z, \omega)=\frac{i}{\rho\left(z_{s}\right) \sqrt{8 \pi r}} e^{-i \pi / 4} \sum_{m=1}^{M} \psi_{m}\left(z_{s}\right) \psi_{m}(z) \frac{e^{i k_{r m} r}}{\sqrt{k_{r m}}}
$$

where $z_{s}$ is the source depth, $k_{r m}$ is the horizontal wavenumber of mode $m$, and $\psi_{m}(z)$ is the mode function of mode $m . k_{r m}$ and $\psi_{m}(z)$ usually depend on $\omega$, the sound speed profile $c(z)$, and seafloor properties, and are obtained by solving the depth-separated wave equation [33, Eq. 5.90]

$$
\frac{\partial^{2} \psi_{m}(z)}{\partial z^{2}}+\left(\left(\frac{\omega}{c(z)}\right)^{2}-k_{r m}^{2}\right) \psi_{m}(z)=0
$$

along with the boundary conditions at the top and bottom of the waveguide, which depend on the acoustic properties of the sea surface and seafloor. Equation 2.2 is a second-order differential equation, for which a discrete number of solutions exist, each one denoted by the mode number $m$.

$k_{r m}$ and $\psi_{m}(z)$ can be calculated analytically in an ideal waveguide [33, Sec. 2.4.4] and semi-analytically in a Pekeris waveguide [33, Sec. 2.4.5]. Semi-analytic solutions exist for other situations as well, such as surface-trapped modes in an $n^{2}$ linear waveguide ([33, Sec. 2.5.1], [11, Sec. 6.6.1]), although the solutions are in terms of non-elementary math functions. (See [9, Sec. 3] and [10, Sec. 4.4.1] for a complete list of known analytic solutions.)

For waveguides with complicated sound speed profiles and non-vacuum seafloors (i.e., fluid or solid), $k_{r m}$ and $\psi_{m}(z)$ do not have exact analytic solutions. They can be calculated numerically using ocean acoustic software such as Kraken [48]. Or 
they can be calculated approximately using the WKB approximation or perturbation theory ([50, Sec. 1.A], [36, Sec. 3.1]). The WKB approximation provides insight into how the environment (SSP, seafloor) affects $k_{r m}$ and $\psi_{m}(z)$, and is discussed in Sec. 4.2 and Appendix B.

\subsubsection{Basic interpretation of normal modes}

Regardless of how the $k_{r m}$ values are calculated, the complex pressure field in Eq. (2.1) is a sum of modes, each one propagating with its own horizontal wavenumber $k_{r m}$. In this thesis we assume the acoustic medium has no attenuation so that all the $k_{r m}$ are real, though many of the concepts presented could likely be extended to the case of mildly attenuating media.

Small changes in the SSP or the bottom boundary conditions (seafloor properties) will usually lead to small changes in the values of the horizontal wavenumbers

and the shapes of mode functions. But because $k_{r m}$ is multiplied by the range $r$ in Eq. (2.1), which is typically on the order of $1000 \mathrm{~m}$, small changes in the $k_{r m}$ lead to large changes in the structure of the complex pressure field. An example of this is illustrated in Figs. 2.4 (b) and (e). For this reason, inversion schemes such as matched field processing that use the complex pressure level as measured at a specific range, depth, and frequency, are extremely sensitive to environmental mismatch. Small modeling errors (be it an incorrect environmental parameter value like the speed of sound in a sediment layer, or an incorrect parametrization of the environment like not modeling enough sediment layers) can prevent an inversion scheme from working correctly.

Another quantity of interest is the scalar acoustic intensity, which is the square of the magnitude of the complex pressure. The acoustic intensity as a function of range can be thought of as an interference pattern between all of the modes [33, Sec. 2.4.4-5], and will be discussed further in Sec. 2.3 .

\subsubsection{Types of modes (terminology)}

Understanding the relationship between a mode's horizontal wavenumber and the SSP is important for understanding the waveguide invariant. A brief summary of the relationship is given here, but more detailed descriptions can be found in [60, 
Sec. 2.9], [33, Secs. 2.5.2 and 5.6], and [11, Sec. 6.7]. The terminology used here is from [33, Sec. 1.4.1] where it was used in the context of acoustic rays.

Surface-Reflected Bottom-Reflected (SRBR) modes are modes that have $\frac{\omega}{c_{\text {seafloor }}}<k_{r m}<\frac{\omega}{c_{\max }}$ where $c_{\max }$ is the maximum sound speed in the watercolumn (not including the seafloor), and $c_{\text {seafloor }}$ is the sound speed of the seafloor. (For a vacuum or rigid seafloor, use $c_{\text {seafloor }}=\infty$ ).

The term non-SRBR will be used to refer to any mode that is not an SRBR mode. Non-SRBR modes always have $\frac{\omega}{c_{\max }}<k_{r m}<\frac{\omega}{c_{\min }}$ where $c_{\min }$ is the minimum sound speed on the watercolumn. Examples of non-SRBR modes are surfacetrapped modes, bottom-trapped modes, and waterborne modes.

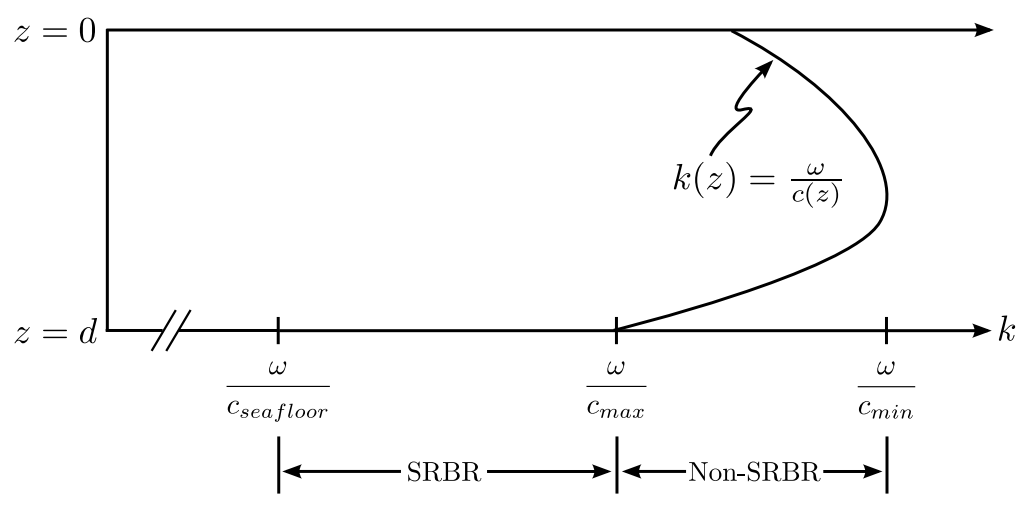

Figure 2.1: Illustration showing the relationship between the sound speed profile, the horizontal wavenumber, and the type of mode. The vertical axis is depth and the horizontal axis is wavenumber. The horizontal axis also represents the horizontal wavenumber $k_{r m}$ of any given mode. Modes with horizontal wavenumbers between $\frac{\omega}{c_{\text {seafloor }}}$ and $\frac{\omega}{c_{\max }}$ are surface-reflected bottom-reflected (SRBR) modes.

\subsubsection{The modal sum}

As shown in Eq. (2.1), the total acoustic pressure is a summation of terms from $M$ normal modes. This modal sum has an interesting property that is essential for understanding the waveguide invariant, but is often not discussed.

In Chuprov's original derivation of the waveguide invariant [18], he states that the acoustic field in a range-independent ocean is typically "composed of a limited number of groups of modes with close [mode] numbers." This subsection explains 
why that is the case, by summarizing the argument presented in [20, Sec. 3.1.6]. ${ }^{1}$

As discussed in [33, Sec. 5.2.1], the mode number $m$ is a monotonic function of $k_{r}$ (this property can also be understood using the WKB approximation in Ch. 4). Thus, a particular range of $k_{r}$ values corresponds to a group of adjacent mode numbers. The quote from Chuprov in the previous paragraph can then be stated as: the complex pressure field at a given range is usually dominated by a group of modes that lie within a small range of $k_{r}$ values - or possibly a few groups of modes with a few distinct ranges of $k_{r}$ values.

Each term of the summation in Eq. (2.1) represents a mode's contribution to the total complex pressure at a fixed range, depth, and frequency. That contribution is a complex number with a magnitude and phase, which can be plotted as a vector in the complex plane. The vector corresponding to the first mode can be plotted from the origin of the complex plane. The vector corresponding to the second mode can plotted from the end of the first mode's vector; the third one plotted from the end of the second one, and so on, as shown in Fig. 2.2 (a). The end of the vector from the $M$-th mode is then the total complex pressure level at the fixed range, depth, and frequency.

From Eq. (2.1), it can be seen that the phase difference between term $(m)$ and term $(m-1)$ is $r\left(k_{r m}-k_{r(m-1)}\right)$. Groups of modes that approximately satisfy the equation

$$
r\left(k_{r m}-k_{r(m-1)}\right)=2 \pi N
$$

where $N$ is an integer will all have approximately the same phase and thus will add constructively (they will all be pointing in the same direction in the complex plane); all other modes will be distributed in "random" directions in the complex plane and will approximately cancel each other out, roughly speaking. This argument is similar to the argument used to justify the stationary phase method of integration.

One can write Eq. (2.3) as

$$
\frac{\partial k_{r m}}{\partial m}=\frac{2 \pi N}{r}
$$

to see that the complex pressure level is dominated by a few groups of modes (each

\footnotetext{
${ }^{1}[18]$ actually referenced [20, Ch. 2], but that was likely an error because the relevant material appears to be in $[20, \mathrm{Ch} .3]$
} 
(a)

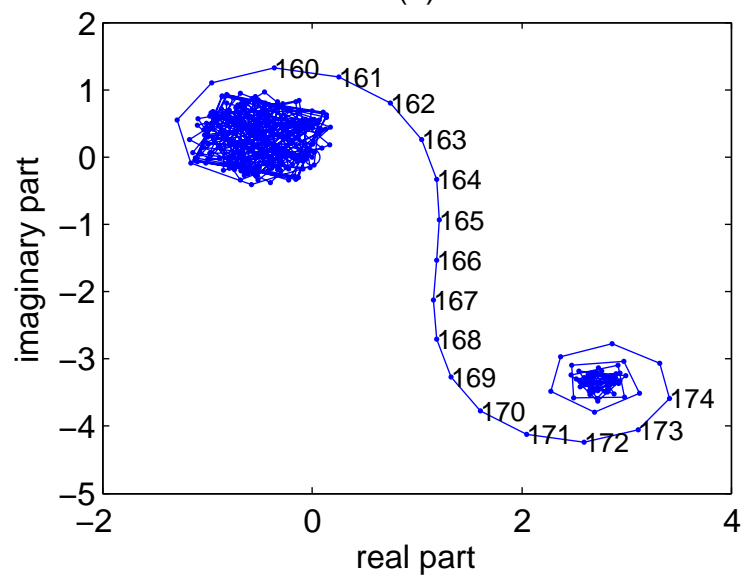

(b)

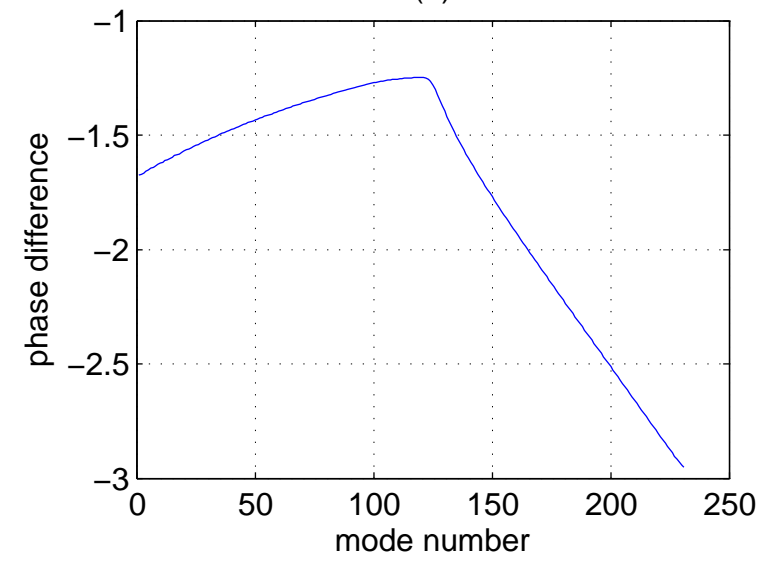

Figure 2.2: (a) Graphical representation of the mode summation for a fixed range, depth and frequency. This plot shows the complex pressure in the deep-water waveguide in Fig. 2.6 at a range of $80 \mathrm{~km}$ and $100 \mathrm{~Hz}$. Each line segment represents the contribution from a single mode number (labeled), beginning with the first modes at $(0,0 i)$ and ending with the last mode about $(2.75,-3.25 i)$. The total complex acoustic pressure is primarily determined by modes 160 to 170. (b) A plot of the phase difference between terms of the modal summation: $r\left(k_{r m}-k_{r(m-1)}\right) /(2 \pi)$. The phase difference for modes 160 to 170 is approximately -2 , an integer, so those modes all have approximately the same phase (direction in the complex plane) and dominant the mode sum. 
one corresponding to a particular value of $N$ ) along the curve of $k_{r}$ versus $m$. Figure 2.2 (b) is a plot of $r\left(\frac{\partial k_{r m}}{\partial m}\right) /(2 \pi)$ versus $m$ for a deep-water waveguide at $80 \mathrm{~km}$ in range.

The concepts in the present subsection are illustrated in more detail, and with more mathematical rigor in [20, Sec. 3.1.6], which also discusses how this interpretation of the modal sum relates to ray theory.

\subsection{The range-frequency waveguide invariant}

\section{Historical context and philosophical note}

The Russian literature on ocean acoustics is noticeably different than the Western (i.e., American and European) literature. Each group - Russian and Western has a slightly different way of thinking about ocean acoustics, and each way has its own strengths and weaknesses.

One of the lessons learned from this thesis was that much can be gained by drawing from the strengths of the two different ways of thinking about ocean acoustics, which complement each other very well. For this reason, this author often lists several references for a single concept.

The waveguide invariant, the topic of this thesis, was discovered by a Russian acoustician named S.D. Chuprov in 1982 [18]. Because of the Cold War between the United States and Russia, researchers in the United States (and presumably other Western countries) did not know about the waveguide invariant until the 1990s.

Chuprov's original paper on the waveguide invariant, Ref. [18], has been translated to English. ${ }^{2}$ Chuprov's paper [18] is extremely dense - covering much material not even mentioned in this thesis - and requires a very deep understanding of ocean acoustics.

\subsubsection{Range-frequency striations}

This thesis uses the term "waveguide invariant" to refer to the range-frequency waveguide invariant. In other literature, "waveguide invariant" sometimes refers

\footnotetext{
${ }^{2}$ This author obtained a copy from the library at the NATO Undersea Research Centre.
} 
to a slightly more general concept that describes not only changes in range and frequency, but also changes environmental parameters such as waveguide depth ([18], [34, Sec. 2.4.6]).

Sections 2.3.1 - 2.3.3 of this thesis draw heavily from [34, Sec. 2.4.6] and [11, Sec. 6.7.2], both of which have excellent derivations and discussions of the waveguide invariant. The derivation in this chapter attempts to complement those two sources by presenting the concepts in a slightly different manner, and by explicitly showing many of the intermediate steps of the derivation. This section uses normal modes to describe the waveguide invariant, but the waveguide invariant can also be described using ray theory (see Appendix A.2). Also, Chapter 6 presents some original research relating the waveguide invariant to wavenumber integration.

We start out with an empirical observation: A 2-d plot of acoustic intensity in a waveguide as a function of range and frequency $\left(I(r, \omega)=|p(r, \omega)|^{2}\right)$ for fixed source and receiver depths often contains striations. This plot is what one would obtain if he or she plotted a spectrogram from an acoustic receiver being towed directly away from a broadband acoustic source in a range independent waveguide.

$I(r, \omega)$ versus $r$ for a fixed $\omega$ is the intermodal interference pattern discussed in [33, Sec. 2.4.4-5], so the striations in $I(r, \omega)$ are a result of how that intermodal interference pattern changes with frequency. The striations in $I(r, \omega)$ will be referred to as waveguide invariant striations, and can be observed under a wide range of environmental conditions.

A simple example of these waveguide invariant striations is shown in Fig. 2.3 (a), which shows the simulated acoustic intensity in a Pekeris waveguide (source at $z_{s}=40 \mathrm{~m}$, receiver at $z=20 \mathrm{~m}, c_{\text {water }}=1500 \mathrm{~m} / \mathrm{s}, c_{\text {bottom }}=1700 \mathrm{~m} / \mathrm{s}$, $\rho_{\text {bottom }}=1750 \mathrm{~kg} / \mathrm{m}^{3}$ — but all Pekeris waveguides will have similar striations). More complicated examples are shown in Figures 2.4 (c) and (d), which show the waveguide invariant striations for two shallow-water waveguides with non-uniform sound speed profiles. Figure 2.5 shows striations from experimental data collected in a shallow-water waveguide (see Ch. 3 for details). Figure 2.6 shows simulated waveguide invariant striations for a deep-water waveguide with a Munk sound speed profile. These striations from a deep-water waveguide are different looking than those from shallow-water waveguides shown in Figs. 2.3 and 2.4, and will 
be discussed later.

These plots show that similar striation patterns appear in many different shallowwater range-independent environments (with the deep-water waveguide having a different pattern). In shallow water, the striation pattern is often not strongly affected by the details of the sound speed profile or the seafloor properties: thus the term waveguide invariant.

The acoustic pressure in a range-independent waveguide can be written as [see Eq. (2.1)]

$$
p(r, \omega) \propto \sum_{m} \psi_{m}\left(z_{s}\right) \psi_{m}(z) \frac{e^{i k_{r m} r}}{\sqrt{k_{r m} r}},
$$

keeping in mind that $k_{r m}$ and $\psi_{m}(z)$ depend on $\omega$. Note that although the pressure depends on $z$, as well as $r$ and $\omega$, the $z$ dependence is not explicitly written on the left-hand-side of Eq. (2.5) because the $z$ dependence is not important for this derivation. Define:

$$
A_{m}=\psi_{m}\left(z_{s}\right) \psi_{m}(z) \frac{1}{\sqrt{k_{r m} r}}
$$

The pressure can then be written as

$$
p(r, \omega) \propto \sum_{m} A_{m} e^{i k_{r m} r}
$$

$A_{m}$ is a slowly varying function of $r$ and $\omega$ compared to how quickly the exponential term in Eq. (2.5) varies with $r$ and $\omega$, and so $A_{m}$ 's dependence on $r$ and $\omega$ will be ignored. $A_{m}$ can be thought of as the local amplitude in the $(r, \omega)$ plane.

The scalar acoustic intensity is the pressure [Eq. 2.7] multiplied by its complex conjugate, which can be written as ${ }^{3}$

\footnotetext{
${ }^{3}$ Assuming $A_{m}$ and $A_{l}$ are real. If $A_{m}$ and $A_{l}$ are complex, then the $A_{m} A_{l}$ in the result must be replaced with $\left|A_{m} A_{l}^{*}+A_{l} A_{m}^{*}\right|$, which is approximately equal to $A_{m} A_{l}$ when the real parts of $A_{m}$ and $A_{l}$ are much larger than the imaginary parts.
} 
(a)

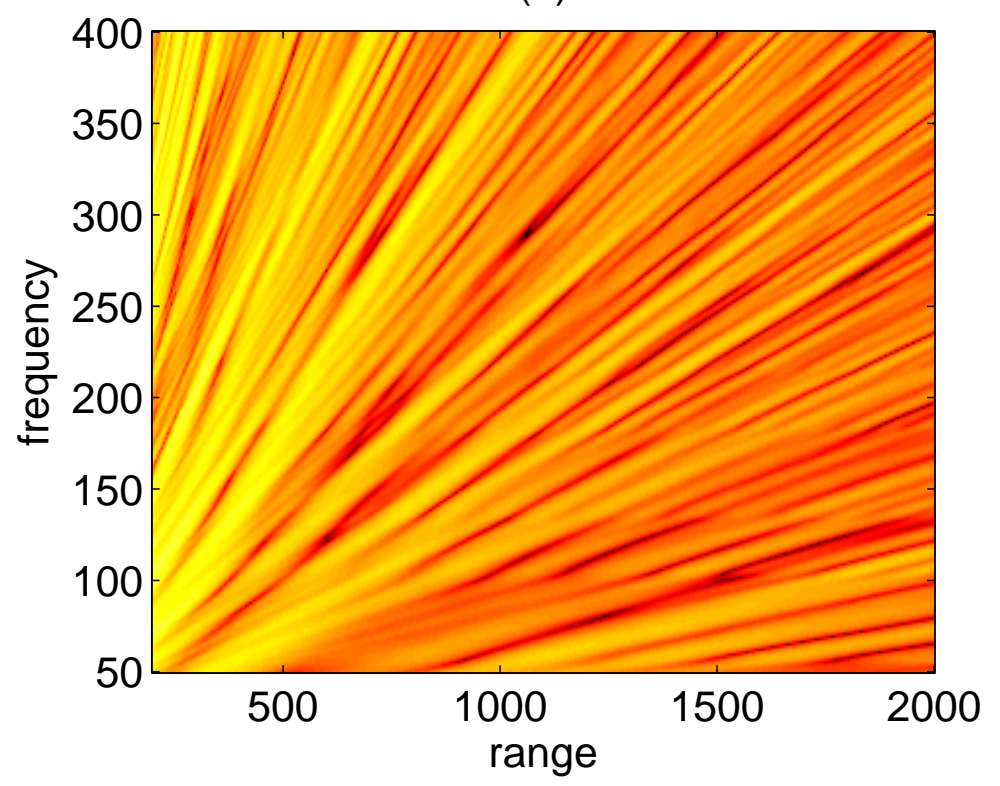

(b)

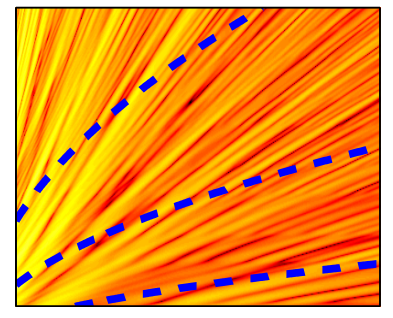

(c)

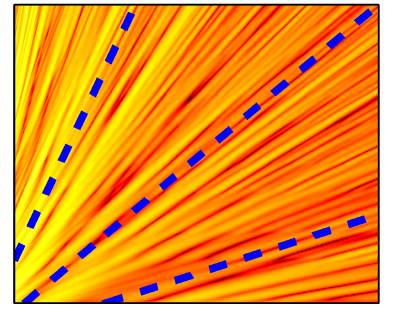

(d)

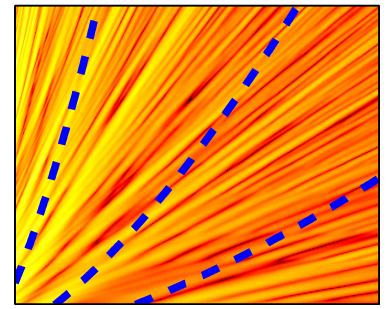

Figure 2.3: (a) Plot illustrating waveguide invariant striations in a simulated Pekeris waveguide. The quantity plotted is acoustic intensity $(\mathrm{dB}$ with an arbitrary reference) versus range and frequency. The striations' slopes (pointing toward the lower left) are described by the waveguide invariant $\beta$. (b) Same as (a), but overlaid with lines corresponding to $\beta=1 / 2$. (c) same as (b) but with lines corresponding to $\beta=1$. (d) same as (b) but with lines corresponding to $\beta=3 / 2$. Note that the striations are best described with $\beta=1$. 
(a)

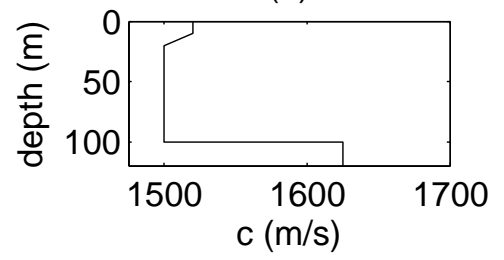

(b)

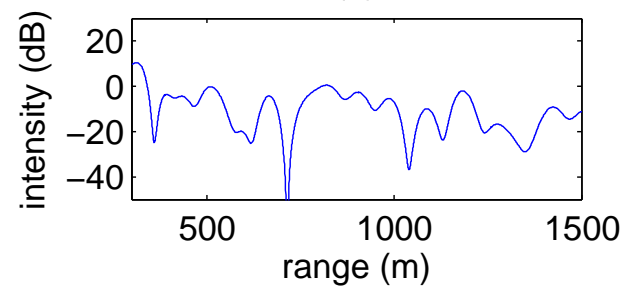

(c)

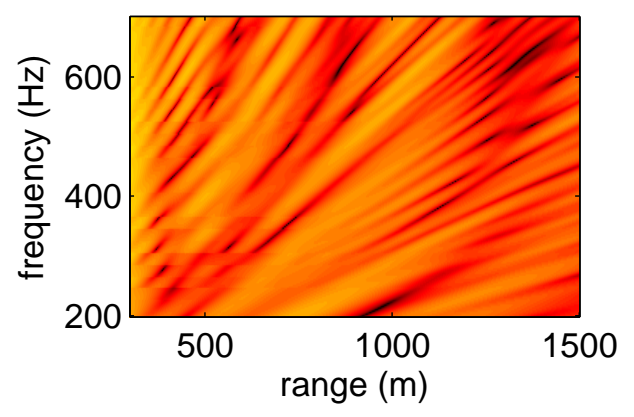

(d)

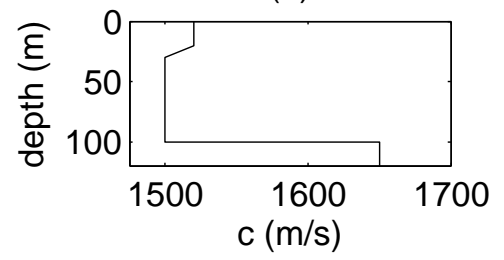

(e)

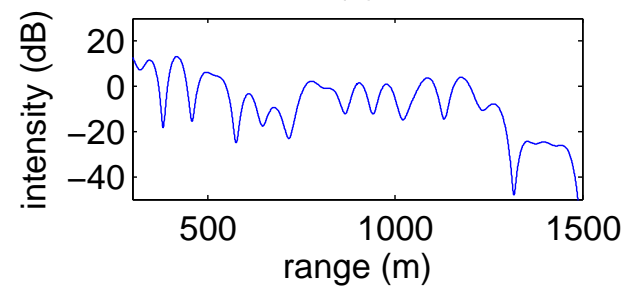

(f)

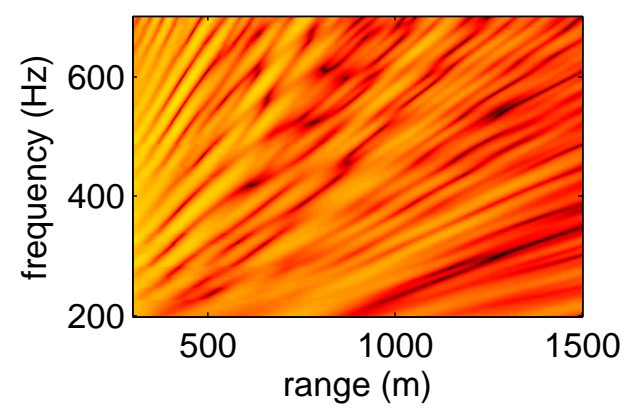

Figure 2.4: Plots illustrating the waveguide invariant for two different simulated shallow-water waveguides, with the properties of one waveguide plotted in the left column and the properties of the other waveguide in the right column. The top row shows the sound speed profiles (including the seafloor). The second row shows the acoustic intensity ( $\mathrm{dB}$ with an arbitrary reference) versus range at $350 \mathrm{~Hz}$, with the source at $37 \mathrm{~m}$ deep and the receiver at $10 \mathrm{~m}$ deep. The plots in the second row illustrate that two waveguides with similar (but not identical) sound speed profiles can have dramatically different acoustic fields. The plots in the third row are acoustic intensity ( $\mathrm{dB}$ with an arbitrary reference) versus range and frequency. Both waveguides, despite their differing sound speed profiles, contain similar-looking striations, the slopes of which are described by the waveguide invariant $\beta$. 


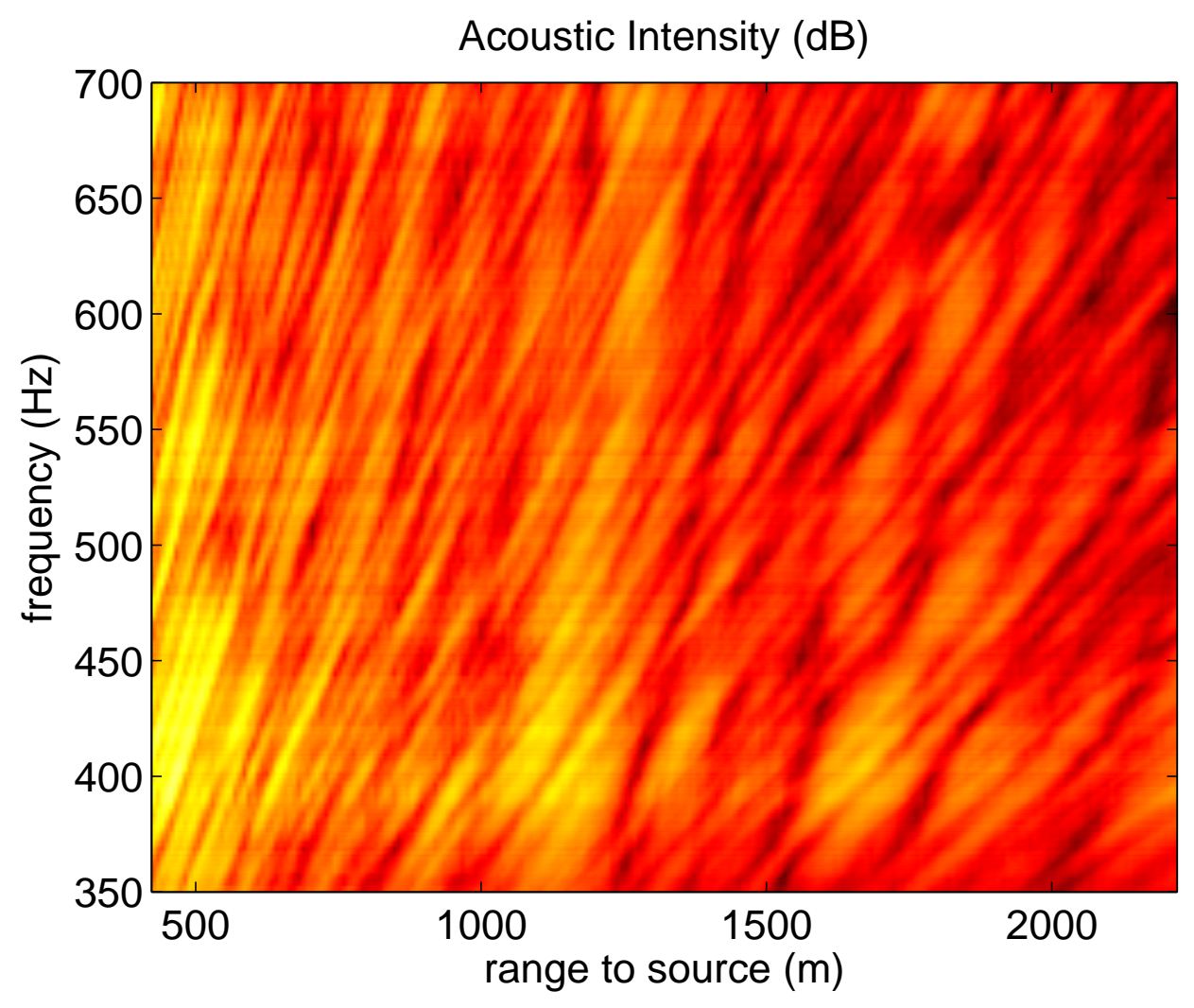

Figure 2.5: Acoustic intensity ( $\mathrm{dB}$ with an arbitrary reference) versus range and frequency illustrating waveguide invariant striations with experimental data from a shallow-water waveguide. 
(a)

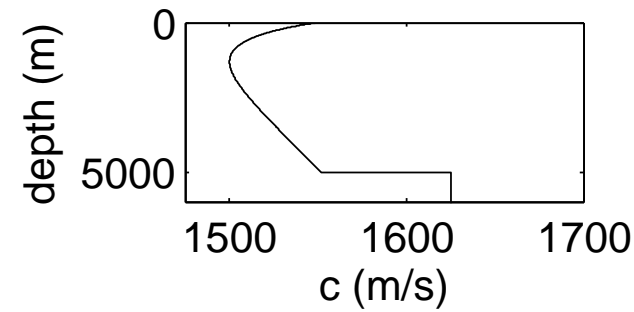

(b)

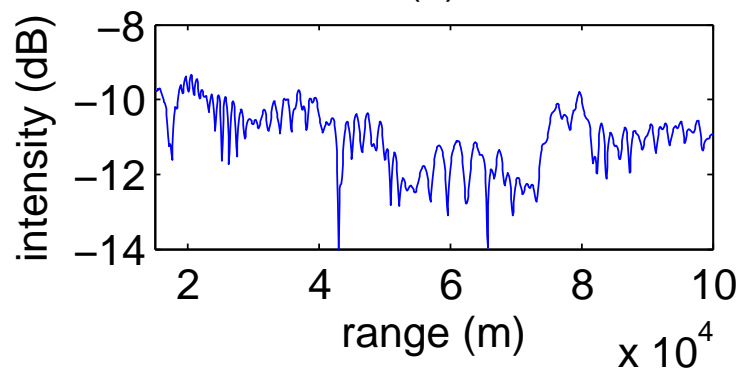

(c)

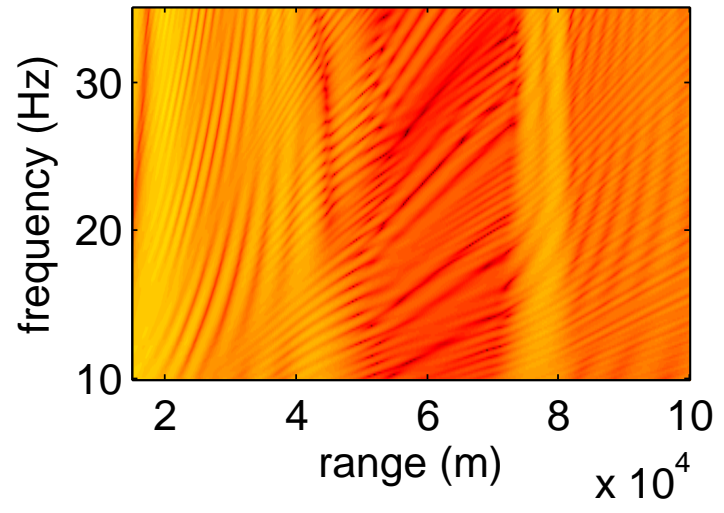

Figure 2.6: Similar to Fig. 2.4, but for a deep-water waveguide with a Munk sound speed profile and plotted with different range and frequency scales. The source is at $300 \mathrm{~m}$ deep and the receiver is $3100 \mathrm{~m}$ deep, so low order modes contribute significantly to the total acoustic intensity. Note that the striation pattern looks qualitatively different than those in Figs. 2.3 (a) and 2.4 (c) and (e). 


$$
\begin{aligned}
I(r, \omega) & =|p(r, \omega)|^{2} \\
& \propto\left(\sum_{m} A_{m} e^{+i k_{r m} r}\right) \cdot\left(\sum_{l} A_{l} e^{-i k_{r l} r}\right) \\
& =\sum_{m} \sum_{l}\left(A_{m} A_{l} \cos \left(\Delta k_{m l} r\right)\right) \\
& =\sum_{q} A_{q}^{2}+\sum_{m, l ; m \neq l} A_{m} A_{l} \cos \left(\Delta k_{m l} r\right)
\end{aligned}
$$

where $m$ and $l$ are the mode number indices, $\Delta k_{m l}=k_{r m}-k_{r l}$ depends on $\omega$, and the exponents have been written as cosines using Euler's formula. Inspection of Eq. (2.10) reveals that the acoustic intensity is a sum of cosines, each cosine resulting from the interference between two modes and having the amplitude of the product of those two modes' amplitudes.

Define:

$$
I_{m l}(r, \omega) \equiv A_{m} A_{l} \cos \left(\Delta k_{m l} r\right)
$$

so that $I_{m l}(r, \omega)$ is the contribution to the total intensity from a single cosine term. A plot of a $I_{m l}(r, \omega)$ versus $\omega$ and $r$, will contain striations, as shown in Fig. 2.7 for $m=4$ and $l=10$.

The slope of those striations can be determined by finding the direction in the $(r, \omega)$ plane for which the intensity does not change. This is done by taking a firstorder two-dimensional Taylor series expansion of $I_{m l}(r, \omega)$ about some point in the $(r, \omega)$ plane, and setting the result equal to zero:

$$
\frac{\partial I_{m l}(r, \omega)}{\partial r} \delta r+\frac{\partial I_{m l}(r, \omega)}{\partial \omega} \delta \omega=0
$$

Then the slope $\frac{\delta \omega}{\delta r}$ (the direction in which $I_{m l}(r, \omega)$ does not change value) can be solved for:

$$
\frac{\delta \omega}{\delta r}=-\frac{\partial I_{m l}(r, \omega)}{\partial r} / \frac{\partial I_{m l}(r, \omega)}{\partial \omega}
$$

From the perspective of a person walking around on the bumpy surface of $I_{m l}(r, \omega)$, Eqs. (2.12) and (2.13) can be stated in words as: If I am at a location 


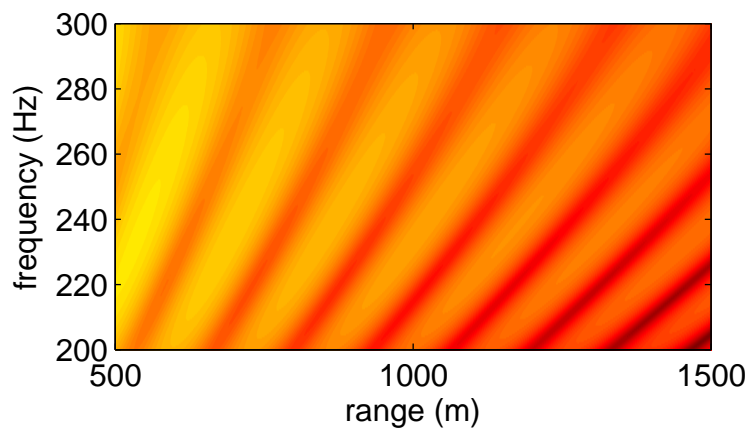

Figure 2.7: A plot of a single cosine term of the acoustic intensity for the waveguide shown in Fig. 2.4 (d), computed using modes 4 and 10.

$(r, \omega)$ and I move in the $r$ direction by some small amount $\delta r$, then how far must I move in the $\omega$ direction $(\delta \omega)$ so that $I_{m l}$ has the same value as where I started? A "striation" is a line or curve in the $(r, \omega)$ plane for which $I_{m l}$ does not change.

Inserting Eq. (2.11) into Eq. (2.13) yields

$$
\frac{\delta \omega}{\delta r}=-\frac{A_{m} A_{l} \cdot\left(\Delta k_{m l}(\omega)\right) \sin \left(\Delta k_{m l}(\omega) r\right)}{r A_{m} A_{l} \cdot\left(\frac{\partial \Delta k_{m l}(\omega)}{\partial \omega}\right) \sin \left(\Delta k_{m l}(\omega) r\right)}=-\frac{\left(\Delta k_{m l}(\omega)\right)}{r\left(\frac{\partial \Delta k_{m l}(\omega)}{\partial \omega}\right)}
$$

We then define the waveguide invariant parameter for these two modes, $\beta_{m l}$, as:

$$
\beta_{m l} \equiv-\frac{1}{\omega} \frac{\Delta k_{m l}(\omega)}{\partial \Delta k_{m l}(\omega) / \partial \omega}
$$

The reason for this definition is not immediately obvious, but it turns out to be a useful one. Using Eq. (2.15) and the right hand side of Eq. (2.14), one can write

$$
\frac{\delta \omega}{\delta r}=\beta_{m l} \frac{\omega}{r}
$$

So the slope $\frac{\delta \omega}{\delta r}$ of a striation of a single $I_{m l}$ term is $\beta_{m l}$ times the frequency $\omega$ divided by the range $r$.

But the acoustic intensity $I(r, \omega)$ consists of a sum of $I_{m l}$ terms, and each $\beta_{m l}$ only describes striations in a single $I_{m l}$ term. If all of the $I_{m l}$ terms have $\beta_{m l}$ values that are approximately the same, then each term will have striations 
with approximately the same slopes, and so the total intensity $I(r, \omega)$ will have striations described by a scalar value $\beta$ that is approximately independent of $m$ and $l$. Section 2.3.2 shows that in an ideal waveguide, $\beta_{m l}$ is approximately independent of $m, l$, and $\omega$, for modes far from cut-off.

In more complicated waveguides, however, $\beta_{m l}$ is not independent of $m, l$, and $\omega$. In that case, a more sophisticated view of the waveguide invariant is required in order to understand why there are striations in $I(r, \omega)$ even though $I(r, \omega)$ consists of a sum of $I_{m l}$ terms, each of which has a different $\beta_{m l}$ value (and thus different striations slopes). This more sophisticated view is described in Sec. 2.3.3. But first, Sec. 2.3.2 shows that $\beta_{m l} \approx 1$ in an ideal waveguide.

Note that in Eq. 2.16, one can replace $\omega$ (the temporal frequency in radians per second) with $f$ (the temporal frequency in cycles per second) because a factor of $2 \pi$ will appear on both sides of the equation and will thus cancel out. Also note that Eq. 2.16 is approximate because the dependence of $A_{m}$ on $r$ and $\omega$ has been ignored.

\subsubsection{The waveguide invariant in ideal waveguides}

$\beta_{m l}$ can be calculated analytically in an ideal waveguide (pressure-release top and bottom, iso-speed SSP). Following [11, Sec. 6.7.2], the horizontal wavenumbers are:

$$
k_{r m}=\sqrt{k^{2}-\left(\frac{m \pi}{d}\right)^{2}}
$$

where the total wavenumber $k=\frac{\omega}{c}$, and the vertical wavenumber of mode $m$ is $\frac{m \pi}{d}$. Eq. (2.17) can be written as

$$
k_{r m}=k \sqrt{1-\left(\frac{m \pi}{k d}\right)^{2}}
$$

By letting $x=\left(\frac{m \pi}{k d}\right)^{2}$, we can write Eq. (2.18) as

$$
k_{r m}=k \sqrt{1-x}
$$

If $x \ll 1$ (which means the vertical wavenumbers are much smaller than the total wavenumber, or equivalently that the angle of propagation is close to horizontal), 
then we can Taylor series expand $\sqrt{1-x}$ to

$$
\sqrt{1-x}=1-\frac{x}{2}+O\left(x^{2}\right)
$$

Inserting $x$ in Eq. (2.20) and ignoring $O\left(x^{2}\right)$ terms, we obtain

$$
k_{r m} \approx k\left(1-\frac{1}{2}\left(\frac{m \pi}{k d}\right)^{2}\right)
$$

where we used the approximate sign because we are ignoring all terms of $O\left(x^{2}\right)$ or higher. Under that assumption (and replacing $k$ with $\frac{\omega}{c}$ ),

$$
k_{r m}-k_{r l} \approx \frac{1}{2}\left(\frac{c}{\omega}\right)\left(\frac{\pi}{d}\right)^{2}\left(m^{2}-l^{2}\right)
$$

This can be inserted into Eq. (2.15) to reveal that $\beta \approx 1$ in this case, regardless of the values of $m, l$, and $\omega$ (bearing in mind that this is only valid for modes with small vertical wavenumbers compared to their horizontal wavenumbers). An alternative derivation expresses the same result in terms of the modal propagation angles [18, Eq. 9] [34, Sec. 2.4.6].

Modes far from cut-off in a Pekeris waveguide have horizontal wavenumber differences and frequency dependencies that are similar to modes in an ideal waveguide. To see this, consider a plot of the characteristic equation for a Pekeris waveguide [33, Eq. 2.167] versus horizontal wavenumber, and how that plot would change with frequency. Because of this, modes far from cut-off in a Pekeris waveguide also have $\beta_{m l} \approx 1$.

Experimental and numerical observations suggest that $\beta_{m l} \approx 1$ in many shallowwater waveguides, regardless of their sound speed profiles. Qualitative reasons for this are suggested in [18]; Chapter 4 investigates this in more depth.

\subsubsection{The waveguide invariant non-ideal waveguides}

One way to understand the waveguide invariant in non-ideal waveguides is to manipulate Eq. (2.15) to define $\beta_{m l}$ in terms of the group slowness and phase slowness (the reciprocal of group speed and phase speed, respectively). Group and phase speeds are discussed in [33, Sec. 2.4.4] and [10, Sec. 4.5] (with rather different 
points-of-view).

The phase speed for a mode $m$ is

$$
v_{m}=\frac{\omega}{k_{r m}}
$$

and the group speed is

$$
u_{m}=\frac{\partial \omega}{\partial k_{r m}}
$$

The phase slowness is

$$
S_{p, m}=\frac{1}{v_{m}}
$$

and the group slowness is

$$
S_{g, m}=\frac{1}{u_{m}}
$$

Comparing those definitions with the right-hand-side of Eq. (2.15), one can write:

$$
\beta_{m l}=-\frac{\Delta S_{p, m l}(\omega)}{\Delta S_{g, m l}(\omega)}
$$

where $\Delta S_{p, m l}=S_{p, m}-S_{p, l}$, and analogously for $\Delta S_{g, m l}$. (The last several equations are contained in original waveguide invariant paper, Ref. [18], but the notation and interpretation used in this thesis come from [34, Sec. 2.4.6] and [66].)

The meaning of Eq. (2.27) can be illustrated by a plot of $S_{g}$ versus $S_{p}$ for all the 1 through $M$ modes (at a fixed $\omega$ ), as is done by Ref. [18] and [34, Sec. 2.4.6]. This plot of $S_{g}$ versus $S_{p}$ can be thought of as a functional relationship: $S_{g}$ as a function of $S_{p}$ with both $S_{g}$ and $S_{p}$ being parameterized by the mode number $m$. This function will be denoted as $S_{g}\left(S_{p}\right)$. In fact, under the WKB approximation, it can be shown that $S_{g}$ is a function of $S_{p}$, independent of $\omega$, for modes that do not interact with the seafloor. This is mentioned in Ref. [18] and can be seen in Eq. (4.36) of this thesis.

If we consider only regions of $S_{g}\left(S_{p}\right)$ where $S_{g}\left(S_{p}\right)$ is approximately a straight line (mathematically speaking, take a first order Taylor series expansion of $S_{g}\left(S_{p}\right)$ about some fixed $S_{p}$ value), then $\beta$ is defined in terms of the slope of that line:

$$
\frac{1}{\beta\left(S_{p}\right)} \equiv-\frac{d S_{g}}{d S_{p}}
$$


(a)
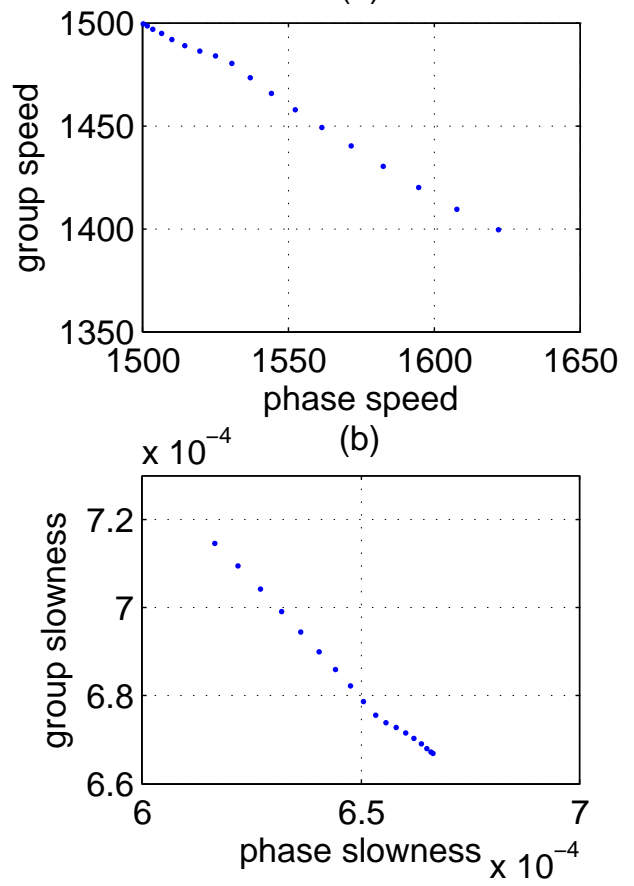

(c)

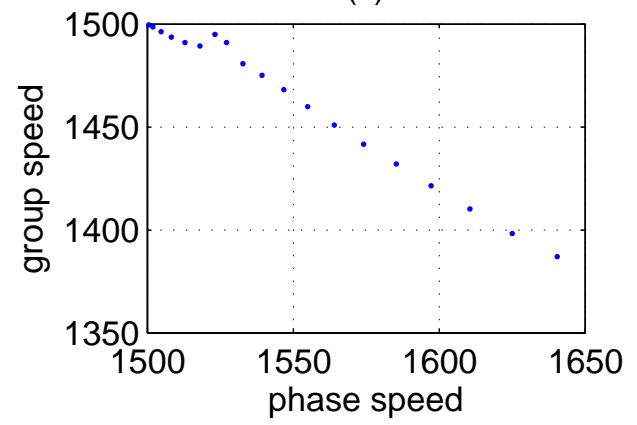

(d)

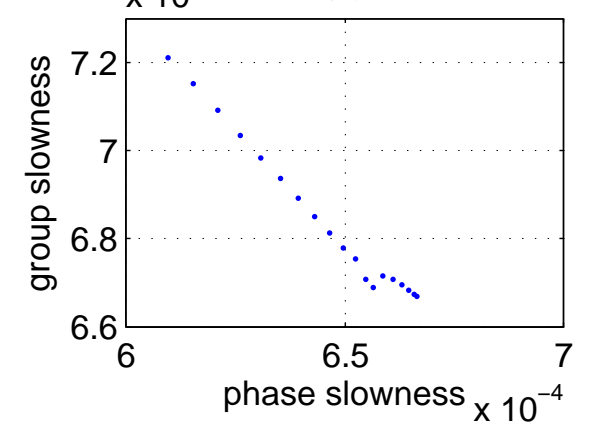

Figure 2.8: The plots in the left column correspond to waveguide in the left column in Fig. 2.4, and the plots in the right column correspond to waveguide in the right column in Fig. 2.4. The top row is a plot of group speed versus phase speed at $350 \mathrm{~Hz}$, with each dot corresponding to a mode. The bottom row is a plot of group slowness versus phase slowness at $350 \mathrm{~Hz}$. The slope of a line connecting points of group slowness versus phase slowness is the reciprocal of the waveguide invariant. 
(a)
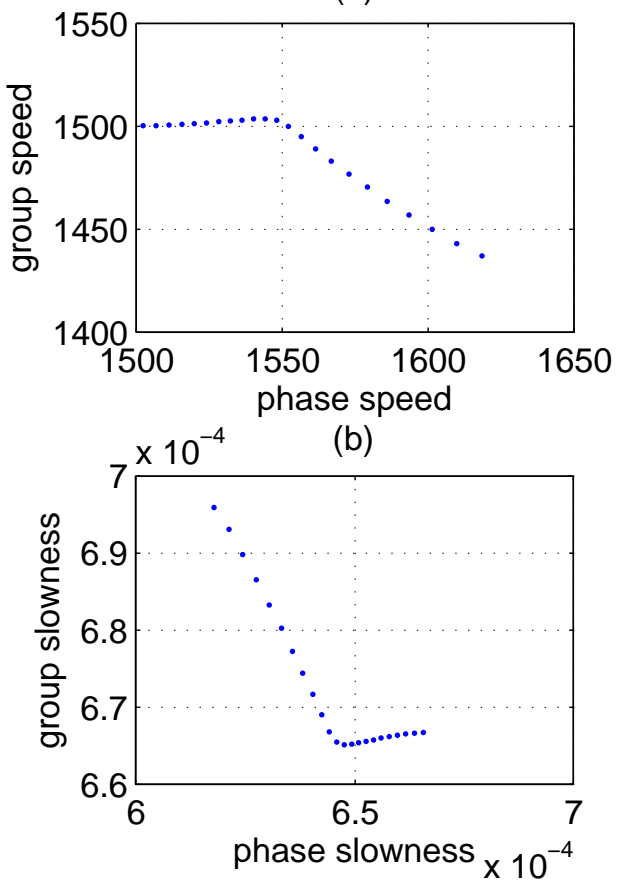

Figure 2.9: Plots of (a) group speed versus phase speed and (b) group slowness versus phase slowness for the deep-water waveguide shown in Fig. 2.6, at $50 \mathrm{~Hz}$. Each dot corresponds to one mode. The slope of a line connecting points in the group slowness versus phase slowness plot is the reciprocal of the waveguide invariant. 
This $\beta$ no longer has an $m l$ subscript because it depends only on the phase slowness $S_{p}$ in $S_{g}\left(S_{p}\right)$, as opposed to depending on the phase slownesses of two distinct modes as does Eq. (2.27). When calculated this way, $\beta$ corresponds to two modes with adjacent modes numbers. $\beta$ may depend on $\omega$ as well, as will be discussed in Ch. 4.

It may seem strange (or even useless) to define $\beta$ using only adjacent modes, given that the acoustic intensity contains components from all mode pairs, including those with $m$ and $l$ values that are not close to each other [see Eq. (2.9)]; each $I_{m l}(r, \omega)$ will have striations according to its $\beta_{m l}$. However, as discussed in Sec. 2.2.3, the acoustic field at fixed range and frequency, is dominated by groups of modes with adjacent mode numbers. That provides justification for defining $\beta$ using only modes with adjacent mode numbers [18]. This author is not aware of any research attempting to quantify the validity of such a justification. But evidently, Eq. (2.28) often provides an accurate description of range-frequency striations.

Figure 2.8 (a) and (c) shows plots of phase speed and group speed for the two shallow-water waveguides discussed earlier in this chapter. All modes with phase speeds less than $1520 \mathrm{~m} / \mathrm{s}$ - the speed of sound at the top of the water column - are non-SRBR mode. An analogous statement can be made for the phase slownesses: all modes with $S_{p}=\frac{k_{r m}}{\omega}>\frac{1}{1520 \mathrm{~m} / \mathrm{s}} \approx 6.57 \mathrm{~s} / \mathrm{m}$ are non-SRBR modes. It can be seen in Fig. 2.8 that the slope of the line connecting non-SRBR modes is significantly different than the slope of the line connecting the SRBR modes. Thus, the non-SRBR modes and the SRBR modes each correspond to significantly different values of $\beta$. However, because there are many more SRBR modes than non-SRBR modes in these cases, the striation pattern observed in $I(r, \omega)$ corresponds mostly to the value of $\beta$ for the $\operatorname{SRBR} \operatorname{modes}(\beta \approx 1)$.

Figure 2.9 is the same as Fig. 2.8, but for the deep-water waveguide shown in Fig. 2.6. In this case, a significant fraction of the modes are non-SRBR modes (which in this case are modes with phase speeds less than about $1550 \mathrm{~m} / \mathrm{s}$ ). That partially explains why the striations in Fig. 2.6 (c) do not look the same as those in Fig. 2.9(c) and (f): non-SRBR modes tend to have different values of $\beta$ than the SRBR modes do.

Furthermore, the slope of the striations in Fig. 2.6 (c) appear to vary depending on the range and frequency, which makes sense given the argument in Sec. 2.2.3: 
different locations in Fig. 2.9 are dominated by different groups of modes, which have different values of $\beta$. This author is not aware of any research directly investigating how the value of $\beta$ observed in the local striations in $I(r, \omega)$ may change with range and frequency (See Sec. 7.3 for a suggestion on how to investigate this).

In many cases, however, the range-frequency striation pattern is well described by a single, dominant value of $\beta$ that is approximately independent of range, frequency,

and mode numbers. In such cases, the striation slopes $\frac{\delta \omega}{\delta r}$ can often be described with the equation

$$
\frac{\delta \omega}{\delta r}=\beta \frac{\omega}{r}
$$

which does not include $\beta$ 's dependence on the mode numbers $m$ and $l$, and the frequency $\omega$ (or alternatively $S_{p}$ and $\omega$ ). Eq. (2.29) is a useful approximation in many situations. However, as pointed out by Rouseff and Spindel in [51], it's important to keep in mind that Eq. (2.29) is an approximation, and that $\beta$ is not always equal to a single value.

Eq. (2.29) defines the slope of a striation (assuming the striation is perfectly well described by $\beta$ ), and can be integrated to yield the equation for the striation path itself [21, Eq. 20]:

$$
\omega=\omega_{0}\left(\frac{r}{r_{0}}\right)
$$

where $\omega_{0}$ and $r_{0}$ represent the constant of integration, and define a point in the $(r, \omega)$ plane where the striation will go through. When the source and/or receiver are moving, the $r$ and $\omega$ in Eq. (2.30) can be parameterized by time, as shown in [21, Eq. 20] by D'Spain et al.

\subsection{Chapter summary}

The total acoustic intensity is a summation of cosine terms, each representing the contribution from a pair of modes denoted by $m$ and $l$. Each cosine term - each mode pair - has its own $\beta_{m l}$ value that may depend on $\omega$. The total intensity is the addition of all those cosine terms.

Under some circumstances, the total acoustic pressure and thus the total acous- 
tic intensity is dominated by mode pairs that all have similar values of $\beta_{m l}$. This leads to the approximation that $\beta$ is a scalar parameter.

Understanding that the acoustic intensity is a summation of cosines, each of which has different (but possibly similar) $\beta_{m l}$ value, is useful for understanding plots of $I(r, \omega)$. This understanding may also be useful for applying the waveguide invariant concept to other situations such as array processing, time-reversal mirrors, etc.

This chapter reviewed the background material necessary for understanding the original work presented in the rest of this thesis, all of which uses $\beta$ described by normal modes in a range-independent waveguide. However, much more research has been done on the waveguide invariant, including relationships to ray theory and extensions to range- and azimuthally-dependent waveguides. Appendix A is a brief but thorough review of the waveguide invariant literature. 


\section{Chapter 3}

\section{Robust passive range estimation using the waveguide invariant}

The previous chapter explained that the waveguide invariant, $\beta$, describes rangefrequency striations. In this chapter, original work is presented that utilizes rangefrequency striations to estimate the range to an acoustic source by assuming that $\beta=1{ }^{1}$ Because this chapter presents experimental data that has $\beta \approx 1$, this chapter also serves as a motivation for $\mathrm{Ch}$. 4 which investigates why $\beta \approx 1$ in most shallow-water waveguides.

As with the rest of this thesis, mode numbers are denoted by $m$ and $l$. In contrast to the rest of the thesis, this chapter writes the temporal frequency as $f$ (cycles/s) instead of $\omega$ (radians/s): $\omega=2 \pi f$.

\subsection{Introduction}

Most research on acoustic source localization in the ocean has focused on using coherent signal processing techniques such as Matched Field Processing [3] (MFP). While MFP works well in theory and in numerical simulation, it is often not applicable to real-world situations because it requires very accurate knowledge of the

\footnotetext{
${ }^{1}$ This chapter is based on "Robust passive range estimation using the waveguide invariant" by Kevin L. Cockrell and Henrik Schmidt [The Journal of the Acoustical Society of America. May, 2010].
} 
environment (e.g., sound speed profile and acoustic properties of the sea floor) in order to correctly localize the source.

Other source localization methods have been developed that require much less a priori knowledge about the environment by using the waveguide invariant. The waveguide invariant has been applied to estimating the range to acoustic sources in various circumstances using a variety of signal processing schemes such as:

- Estimating the range to a fixed acoustic source from measurements taken by a vertical hydrophone array by analyzing the MFP sidelobe behavior [59].

- Estimating the closest-point-of-approach of a moving source to a single fixed hydrophone using a Hough transform-like technique [56].

- Estimating source trajectories based on range-frequency striations in an array's beamformed output [62].

In this chapter, a technique is developed to estimate the range to a fixed acoustic source from the acoustic intensity as measured over a window of ranges and frequencies, $I(r, f)$. The technique is tested on experimental data that was obtained from an acoustic receiver towed by an Autonomous Underwater Vehicle (AUV) heading directly toward the acoustic source. Previous research papers have used the two-dimensional discrete Fourier transform (2D-DFT) of $I(r, f)$ to estimate the value of the waveguide invariant when the source range was known [51, 66], but did not address how to choose the signal processing parameters. This chapter extends that research to using the 2D-DFT of $I(r, f)$ to perform range estimation, and develops guidelines for choosing the signal processing parameters' values.

The objective of the present work is to investigate the issues related to the signal processing that is required for range estimation using the 2D-DFT of $I(r, f)$ in the context of performing the estimation autonomously (i.e., without requiring human interpretation of any images), and to perform the range estimation on simulated and experimental data. The main results are:

- The minimum bandwidth of the acoustic source and minimum range window of acoustic intensity measurements required for accurate range estimation can be determined from a modest knowledge of the acoustic waveguide parameters before any acoustic measurements are made. 
- A relationship between the signal processing parameters and the ocean-acoustic waveguide parameters can be used to reject much of the noise present in experimental data.

- Range estimation can be performed robustly, requiring very little a priori environmental knowledge (at least, for the data sets analyzed this chapter).

- A range estimate accuracy of approximately $25 \%$ is achieved with the experimental data set used in this chapter.

Section 3.2 discusses the waveguide invariant in the context of passive range estimation. In Sec. 3.3, the range estimation algorithm based on a 2D-DFT is discussed. Section 3.4 applies the algorithm to simulated and experimental data. And finally, Sec. 3.5 gives a summary and conclusion of this chapter.

\subsection{Brief review of range-frequency striations}

Section 2.3 explained that a plot of acoustic intensity versus range and frequency due to a broadband source in a waveguide, $I(r, f)$, will exhibit striations that are described by the waveguide invariant $\beta$. As discussed in Sec. 2.3 the value of $\beta$ is unique for each mode pair, but $\beta$ can sometimes be approximated as being a single value independent of the mode numbers $m$ and $l$, and the frequency $\omega$. Under that approximation, specific value of $\beta$ that will be observed in $I(r, \omega)$ depends on the sound speed profile, the seafloor properties, and the source and receiver locations $[51,21,49,59]$.

Empirical and numerical observations suggest that for mode pairs where both modes are surface-reflecting bottom-reflection (SRBR) modes, $\beta \approx 1$ (this is investigated further Ch. 4). So if the acoustic intensity is dominated by SRBR modes, the $\beta$ observed in the striation pattern will likely be close to 1 . Throughout this chapter, for both the simulated and experimental data, we assume $\beta=1$. The consequences of assuming $\beta=1$ are discussed shortly.

Eq. (2.29) can be used for range estimation by rewriting it as

$$
r=\beta \cdot f \cdot \frac{\delta r}{\delta f},
$$


which allows for one to estimate the range of the acoustic source if one measures the slopes of the striations and assumes a value of $\beta$. The effect of assuming an incorrect value of $\beta$ can been seen in Eq. (3.1). If the true value of $\beta$ is $\beta_{\text {true }}$ and the assumed value is $\beta_{\text {assumed }}$, then the range estimates will be incorrect by a factor of $\beta_{\text {assumed }} / \beta_{\text {true }}$.

In order to estimate the range to the source, one begins by calculating $I(r, f)$ for some range of values of $r$ and $f$. In a simulation one can calculate $I(r, f)$ in the frequency domain using acoustic simulation software. In an experiment one must estimate the power spectrum of a hydrophone's time series at several ranges. In practice, $I(r, f)$ will likely be the spectrogram of a time series of acoustic pressure obtained by moving acoustic an receiver radially towards or away from the acoustic source, as is done in Sec. 3.4.2.

To estimate the source's location, one then must determine the slopes of the striations (or curved paths of the striations, if $\beta \neq 1$ ) in $I(r, f)$. Because of the visually striking relationship between the striation slope and the source's location, a person looking at $I(r, f)$ can estimate the source's location rather easily (see Fig. 2.3. However, the present work focuses on techniques that perform the range estimation autonomously (i.e., without the benefit of having a person to visually interpret $I(r, f)$ or its 2D-DFT).

\subsection{Using the two-dimensional discrete Fourier transform for range estimation}

\subsubsection{Outline of the 2D-DFT technique}

The slope of the striation, $\frac{\delta f}{\delta r}$, at a particular range-frequency combination $(r, f)$ can be inserted into Eq. (3.1) to estimate the range to the source. The technique described in this section to determine the slope of a striation in $I(r, f)$ looks at a small local region (a "window") of $I(r, f)$ and assumes that all of the striations within that window have the same slope. This is similar to what is done in $[4,51$, 66], but in those papers the ranges were much larger than the ranges used in the present analysis, so the slopes of the striations did not change quickly with range. Consequently, those papers did not focus much attention on how to choose the size 
of the window. Because of the short ranges used in the present analysis, the slopes change quickly with range and so much care must be given to the choosing the window size in order to ensure that the slopes do not change too much inside of the window. This issue is discussed in Sec. 3.3.2.

In addition to the striations from the source, $I(r, f)$ will also contain noise which can be partially eliminated by filtering. The spatial cutoff frequencies of the filter are discussed in Sec. 3.3.2.

The range is then estimated based on the slope of the striations in the window. This process is repeated for several windows located on a grid in the $(r, f)$ plane. Each window on the grid will produce one range estimate, all of which can then be averaged obtain a single, robust estimate. Note that the vertical axis of the window is frequency, so it requires that the source be broadband. The horizontal axis of the window is range, and so it requires that the acoustic field is measured along a line emanating radially from the acoustic source.

\subsubsection{Determining local striation angle with the 2D-DFT}

Denote a rectangular window of $I(r, f)$ bounded by $\left(r_{\min }<r<r_{\max }\right)$ and $\left(f_{\min }<f<f_{\max }\right)$ as $I_{\text {win }}(r, f)$. The striations inside of $I_{\text {win }}(r, f)$ will all have approximately the same slope if the window size is sufficiently small.

Several articles have pointed out the relationship between the 2D-DFT of $I_{\text {win }}(r, f)$ and the slope of the striations in $I_{\text {win }}(r, f)[66,4,51,18]$. That relationship forms the basis for the approach used in the present work to determine the local striation angle. The process of determining the local striation angle involves five main steps:

1. Take a two-dimensional discrete Fourier transform (2D-DFT) of $I_{\text {win }}(r, f)$

2. Eliminate regions of the 2D-DFT of $I_{\text {win }}(r, f)$ associated exclusively with noise content.

3. Convert the 2D-DFT of $I_{\text {win }}(r, f)$ to polar coordinates.

4. For several hypothesized striation angles, add up (integrate) all of the components of the 2D-DFT of $I_{\text {win }}(r, f)$ corresponding to that striation angle.

5. The striation angle that has the most "energy" is then the estimate. 
These steps are illustrated in Fig. 3.1 and are described in detail in the following subsections. The steps are related to the Radon Transform and the FourierSlice Theorem [35]. If one were to skip the 2nd step, then the steps could be performed with a Radon Transform using the Fourier-Slice Theorem. ${ }^{2}$ But because step 2 eliminates components of $I_{\text {win }}(r, f)$ above particular spatial frequencies, the present analysis works directly with the 2D-DFT of $I_{\text {win }}(r, f)$.

\section{Interpretation of the two-dimensional discrete Fourier transform}

$I_{\text {win }}(r, f)$ has striations whose slope needs to be determined in order to estimate the source's range. Fig. 3.1(a) shows an example $I_{\text {win }}(r, f)$. Denote the magnitude of the 2D-DFT of $I_{\text {win }}(r, f)$ as $I_{2 \mathrm{DF}}\left(k_{r, \text { image }}, k_{f, \text { image }}\right)$ :

$$
I_{2 \mathrm{DF}}\left(k_{r, \text { image }}, k_{f, \text { image }}\right) \equiv\left|\iint I_{\text {win }}(r, f) e^{-j 2 \pi\left(k_{r, \text { image }} r+k_{f, \text { image }} f\right)} d r d f\right|
$$

where $k_{r \text {,image }}$ and $k_{f \text {,image }}$ do not refer to the acoustic wavenumbers, but rather to the wavenumbers of the "image" $I_{\text {win }}(r, f)$. Hence, $k_{r \text {,image }}$ and $k_{f \text {,image }}$ will be referred to as the image wavenumbers. They are the horizontal and vertical axis, respectively, of Fig. 3.1(b). In Sec. 3.3.2 the relationship between $k_{r \text {,image }}$ and $k_{f \text {,image }}$ and the acoustic horizontal wavenumbers of the modes propagating in the waveguide will be derived.

In practice one has a discrete (sampled) version of $I(r, f)$, so the two-dimensional Fourier transform is implemented as a two-dimensional discrete Fourier transform. The direction of the mainlobe originating from the origin of $I_{2 \mathrm{DF}}\left(k_{r \text {,image }}, k_{f, \text { image }}\right)$ is perpendicular to the slope of the striations in the window of $I(r, f)$. An example is shown in Figs. 3.1(a) and 3.1(b). (They do not appear exactly perpendicular because of the different aspect ratios of the figures.)

If one thinks of $I_{\text {win }}(r, f)$ as an image, ignorant of the fact that it represents power spectrum of an acoustic field, then its 2D-DFT, $I_{2 \mathrm{DF}}\left(k_{r \text {,image }}, k_{f \text {,image }}\right)$, can be interpreted as a decomposition of $I_{\text {win }}(r, f)$ into "cosine" image basis functions, each with a unique image wavenumber $[7,12]$.

\footnotetext{
${ }^{2}$ The author thanks an anonymous reviewer for The Journal of the Acoustical Society of America for pointing this out.
} 
The acoustician may gain insight by noting that the basis functions of a 2DDFT of an image look like two-dimensional plane waves with the time-dependence removed: $\exp \left(i\left(k_{x} x+k_{y} y\right)\right)$.

Each pixel of $I_{2 \mathrm{DF}}\left(k_{r, \text { image }}, k_{f, \text { image }}\right)$ represents a single basis function with image wavenumbers of $k_{r \text {,image }}$ and $k_{f \text {,image }}$ (horizontal and vertical image wavenumbers, respectively, in Fig. 3.1(a)). One may find this easier to understand if she or he ignores that $r$ and $f$ represent range and frequency, and instead thinks of them simply as labels for the $x$ and $y$ axes of Fig. 3.1(a).

To relate $k_{r \text {,image }}$ and $k_{f \text {,image }}$ to striation angles, one can interpret each combi-

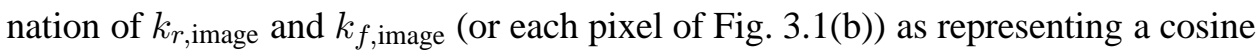
basis function at a particular angle with a particular period (striation width).

\section{Application of the 2D-DFT to determine striation angle}

The waveguide invariant as shown in Eq. (2.29) makes a statement only about the slopes of the striations; it says nothing about the distance between the striations. From an image processing perspective, the waveguide invariant makes a statement about the angle of the basis functions comprising $I_{\text {win }}(r, f)$; it says nothing about the period (striation width) of those image basis functions. But as Chuprov points out in his original derivation of the waveguide invariant, one can calculate the minimum striation width using only modest information about the waveguide [18]. This allows one to filter out noise by only including components of $I(r, f)$ with image wavenumbers less than the maximum expected due to the acoustic source of interest, as will be discussed in Sec. 3.3.2.

To determine the angle of the striations in $I_{\text {win }}(r, f)$ we first remove the mean of $I_{\text {win }}(r, f)$ and then take its 2D-DFT to obtain $I_{2 \mathrm{DF}}\left(k_{r \text {,image }}, k_{f \text {,image }}\right)$. Then we transform $I_{2 \mathrm{DF}}\left(k_{r \text {,image }}, k_{f, \text { image }}\right)$ from Cartesian coordinates to polar coordinates:

$$
I_{2 \mathrm{DF}}\left(k_{r, \text { image }}, k_{f, \text { image }}\right) \Rightarrow I_{2 \mathrm{DF}}(\theta, K)
$$

where

$$
\theta=\arctan \left(k_{f, \text { image }} / k_{r, \text { image }}\right), \quad K=\sqrt{k_{r, \text { image }}^{2}+k_{f, \text { image }}^{2}} .
$$

Note that it is legitimate to add $k_{r \text {,image }}$ and $k_{f \text {,image }}$ together because they are dimensionless, as they are the result of the 2D-DFT. However throughout most of the 
present work $k_{r \text {,image }}$ and $k_{f, \text { image }}$ are "re-dimensionalized" based on the sampling used in the 2D-DFT, just as is typically done with power spectra based on discretely sampled temporal waveforms.

Nearest-neighbor interpolation is used to do the coordinate transform. An example $I_{2 \mathrm{DF}}(\theta, K)$, is shown in Fig. 3.1(c).

If one places bounds on the possible ranges to the acoustic source, one can use Eq. (2.29) to put bounds on the angles that could have striations due to the waveguide invariant. The present analysis assumes the source was between 100 meters and 5,000 meters. Typically this only eliminates a few degrees (e.g., angles of 3 to 87 degrees, instead of 0 to 90 degrees), but the striation angle finding algorithm benefits from this because it occasionally incorrectly chooses very steep or very shallow angles as the dominant striation angle.

$$
\begin{aligned}
& \theta_{\min }=\arctan \left(\frac{\text { maximum frequency in window }}{\text { minimum range to search }}\right) \\
& \theta_{\max }=\arctan \left(\frac{\text { minimum frequency in window }}{\text { maximum range to search }}\right)
\end{aligned}
$$

Interpolation isn't strictly necessary to obtain the value of $I_{2 \mathrm{DF}}(\theta, K)$ at an arbitrary $(\theta, K)$. One can evaluate the 2D-DFT of a sampled version of $I(r, f)$ at arbitrary $k_{r \text {,image }}$ and $k_{f \text {,image }}$ values, analogous to a discrete time Fourier transform for a discrete time series. However, doing this is computationally intensive because one cannot utilize the Fast Fourier Transform algorithm, and the present analysis did not suggest a noticeable increase in striation-angle finding performance when doing this to avoid interpolation.

$I_{2 \mathrm{DF}}(\theta, K)$ is then integrated along the $K$ direction (the $y$-axis in Fig. 3.1(c)) to add up all the components of the image with a particular striation angle (remembering that $I_{2 \mathrm{DF}}(\theta, K)$ will already have been spatially filtered to eliminate high frequency noise).

$$
E(\theta)=\int I_{2 \mathrm{DF}}(\theta, K) d K
$$

$E(\theta)$ approximately represents the amount of energy (in an image processing sense, not in an acoustic sense) in $I_{2 \mathrm{DF}}\left(k_{r \text {,image }}, k_{f \text {,image }}\right)$ of striations at a particular angle in $I(r, f)$. A plot of an $E(\theta)$ is shown in Fig. 3.1(d). In this chapter, we 
assume $\beta=1$ and use $E(\theta)$ to estimate the range. However, other researchers have modeled $\beta$ as a distribution (see Sec. 2.3.3) and used $E(\theta)$ to estimate that distribution [51].

Note that a proper change of variables would give an extra factor of $K$ on the right hand side of Eq. 3.3, as noted in [51]. Because the present analysis does not have that extra factor of $K$, lower values of $K$ are weighted more heavily. The precise meaning of this can be seen by looking at the mapping between pixels in Fig. 3.1(b) and Fig. 3.1(c). This was done because it led to better estimates of the striation angle.

Finally, the angle corresponding to the maximum value of $E(\theta)$ is the most dominant angle in the basis functions comprising $I_{\text {win }}(r, f)$. The angle of the striations is perpendicular to the angle of the basis function:

$$
\theta_{\text {striation }}=\underset{\theta}{\arg \max } E(\theta)+\pi / 2
$$

An $I_{2 \mathrm{DF}}\left(k_{r, \text { image }}, k_{f, \text { image }}\right)$ obtained from experimental data will have noise at

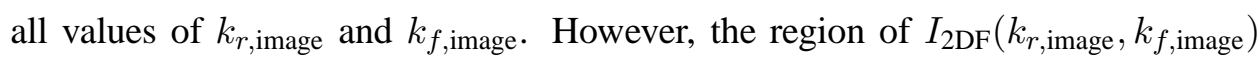
which is important for the slope estimation (the signal of interest) lies mostly at lower values of $k_{r \text {,image }}$ and $k_{f \text {,image }}$. It was found that even at very high signalto-noise ratios, the noise can significantly deteriorate the slope estimate because the maximum value of $k_{r \text {,image }}$ and $k_{f \text {,image }}$ represented $I_{2 \mathrm{DF}}\left(k_{r \text {,image }}, k_{f \text {,image }}\right)$ can be arbitrarily large depending on how finely $I(r, f)$ was sampled in range and frequency. The effect of the noise can be reduced significantly by limiting the region of integration of Eq. (3.3) to $\left(-k_{r \text {,image,max }}<k_{r \text {,image }}<k_{r \text {,image,max }}\right)$ and $\left(0<k_{f, \text { image }}<k_{f \text {,image,max }}\right)$. The next section will demonstrate how to choose $k_{r, \text { image,max }}$ and $k_{f, \text { image,max }}$. 
(a) $I_{\text {win }}(r, \omega)$

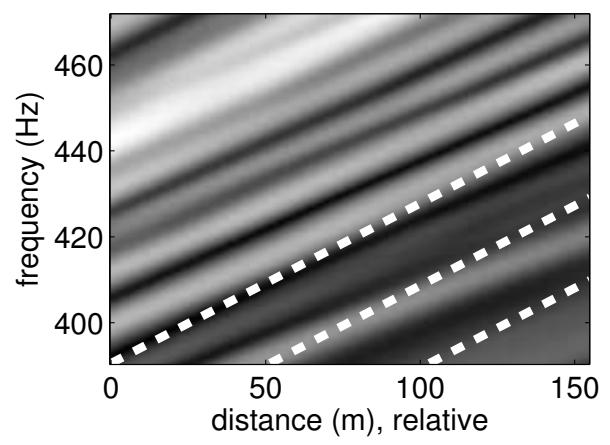

(c) $\mathrm{I}_{2 \mathrm{DF}}(\theta, \mathrm{k})$

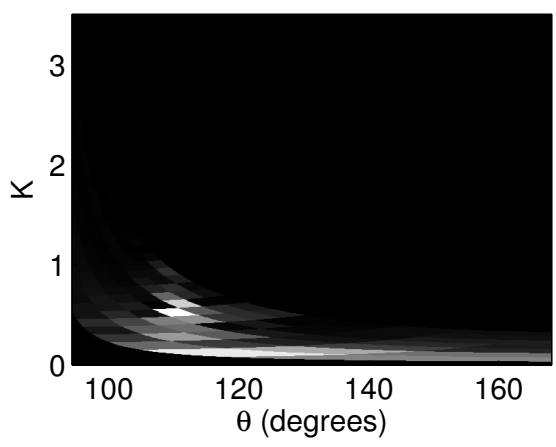

(b) $\mathrm{I}_{2 \mathrm{DF}}\left(\mathrm{k}_{\mathrm{r} \text {,image }}, \mathrm{k}_{\mathrm{f} \text {,image }}\right)$ (Zoomed In)

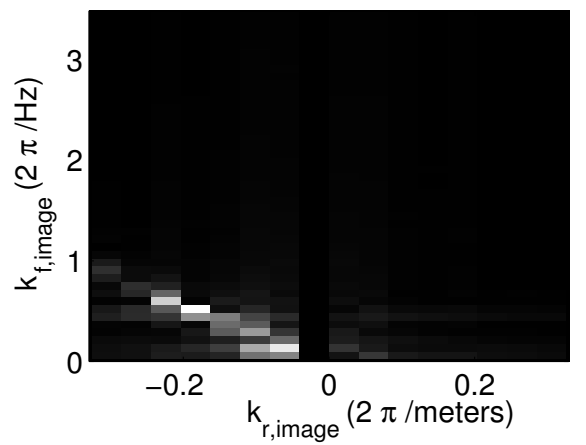

(d) $E(\theta)$

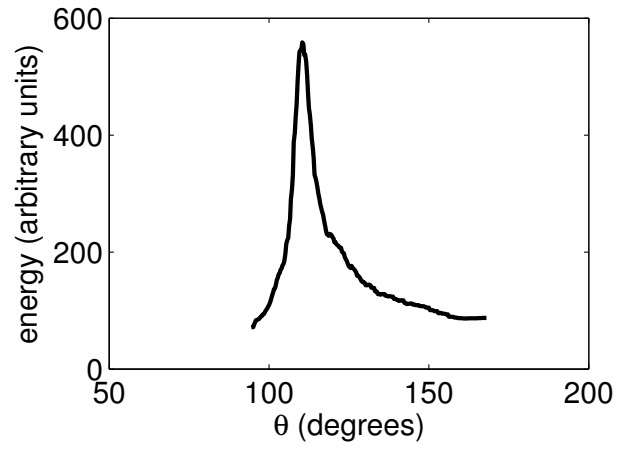

Figure 3.1: The four steps used to determine the angle of the striations in $I_{\text {win }}(r, f)$. Part (a) shows $I_{\text {win }}(r, f)$, a window of $I(r, f)$. The two-dimensional Fourier transform of $I_{\text {win }}(r, f)$ is then taken resulting in $I_{2 \mathrm{DF}}\left(k_{r, \text { image }}, k_{f, \text { image }}\right)$, shown in part (b) (using the bounds described in Sec. 3.3.2). $I_{2 \mathrm{DF}}\left(k_{r \text {,image }}, k_{f \text {,image }}\right)$ is then converted into polar coordinates, $I_{2 \mathrm{DF}}(\theta, K)$, shown in part (c). $I_{2 \mathrm{DF}}(\theta, K)$ is then integrated along $K$ to produce $E(\theta)$, shown in part (d). The angle corresponding to the peak of $E(\theta)$ is then the estimated angle of the striation. White dotted lines corresponding to the estimated striation angle are then plotted in part (a) for visual comparison. 


\section{Upper Bounds on $k_{r, \text { image }}$ and $k_{f, \text { image }}$}

[18] showed that one can relate the environmental parameters of the waveguide to the maximum rate at which $I(r, f)$ can oscillate in $r$ and in $f$. In this subsection, we reproduce the results from [18] in the context of the problem at hand - source range estimation in a shallow-water waveguide. Determining an upper bound on the rate at which $I(r, f)$ oscillates in $r$ and in $f$ is equivalent to determining an upper bound on the $k_{r \text {,image }}$ and $k_{f \text {,image }}$ due to the acoustic source of interest. Any

components of $I_{2 D F}\left(k_{r \text {,image }}, k_{f \text {,image }}\right)$ above some $k_{r \text {,image, max }}$ and $k_{f \text {,image,max }}$ can be regarded as noise because they cannot be due to the source of interest.

In order to exclude as much noise as is possible, the integration in Eq. (3.3) of $I_{2 \mathrm{DF}}\left(k_{r, \text { image }}, k_{f \text {,image }}\right)$ will be bounded by $k_{r \text {,image,max }}$ and $k_{f \text {,image, max }}$. An example of this region is shown in Fig. 3.2. Note that applying these bounds on the is approximately equivalent to low-pass filtering the image.

According to Eq. (2.9), the acoustic intensity is a sum of cosines. Each cosine term has an image wavenumber in the $r$ direction of

$$
k_{r, \text { image }, m l}=\frac{\partial\left(\Delta k_{m l}(f) r\right)}{\partial r}=\Delta k_{m l}(f)
$$

and an image wavenumber in the $f$ direction of

$$
k_{f, \text { image }, m l}=\frac{\partial\left(\Delta k_{m l}(f) r\right)}{\partial f}=\frac{r \partial\left(\Delta k_{m l}(f)\right)}{\partial f}
$$

(where $\Delta k_{m l}=k_{r m}-k_{r l}$ ).

An upper bound on $k_{r \text {,image, } m l}$ in $I(r, f)$ can be determined (to within the approximations used when deriving the waveguide invariant) by calculating the largest possible value of $\Delta k_{m l}(f)$. All non-zero values in $I_{2 \mathrm{DF}}\left(k_{r \text {,image }}, k_{f \text {,image }}\right)$ with $k_{r \text {,image }}$ image wavenumbers above the maximum value of $\Delta k_{m l}(f)$ are likely due to noise, and can be excluded from the integration in Eq. (3.3). For all ocean acoustic waveguides, the horizontal wavenumbers are bounded by [10, Eq. 4.5.19]

$$
\left[\frac{2 \pi f}{c_{\max }}, \frac{2 \pi f}{c_{\min }}\right]
$$

where $c_{\max }$ and $c_{\min }$ are the respective minimum and maximum sound speeds that 


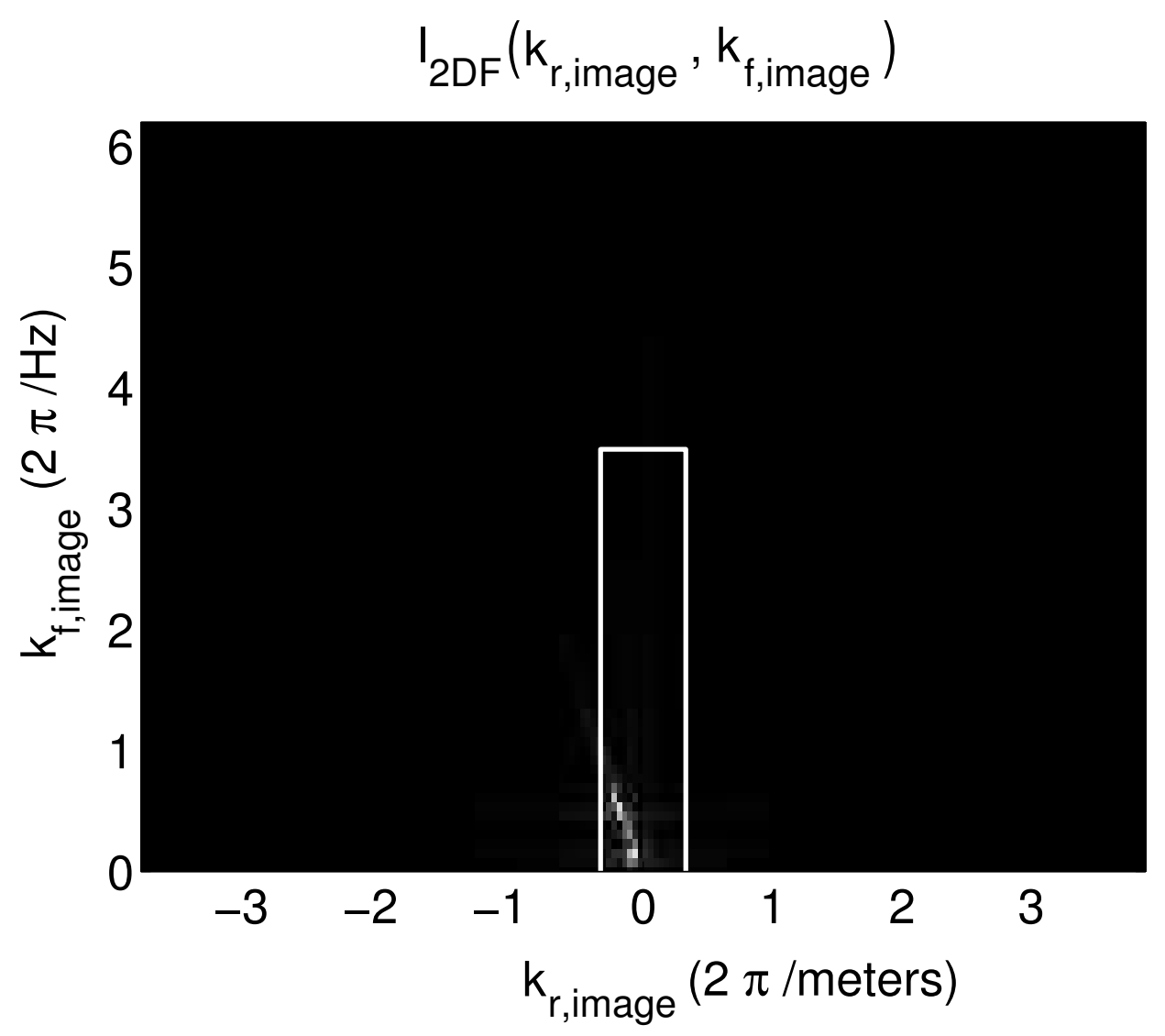

Figure 3.2: $I_{2 \mathrm{DF}}\left(k_{r \text {,image }}, k_{f \text {,image }}\right)$ showing the full range of $k_{r \text {,image }}$ and $k_{f \text {,image }}$, with white lines illustrating the bounds described in Sec. 3.3.2. This figure illustrates why it is essential to limit the region of integration in Eq. (3.3). Even if the noise outside the white lines is at a low level, it can dominate the integral in Eq. (3.3) because of the large ratio of outer to inner areas separated by the white lines. The inner area is what is shown in Fig. 3.1(b). 
occur in the environment. Thus

$$
k_{r, \text { image } \max }=2 \pi f\left(\frac{1}{c_{\min }}-\frac{1}{c_{\max }}\right) .
$$

To determine and upper bound on $k_{f, \text { image, } m l}$, we use Eq. (3.6) and replace $f$ with $\omega /(2 \pi)$, and note that $\frac{\partial k_{m}}{\partial \omega}$ is the reciprocal of the group speed of mode $m$. The maximum and minimum group speeds are approximately bounded by fastest and slowest media in the waveguide, so

$$
k_{f, \text { image } \max }=2 \pi r\left(\frac{1}{c_{\min }}-\frac{1}{c_{\max }}\right) .
$$

These upper bounds are used to limit the region of integration in Eq. (3.3). Using Eqs. (3.9) and (3.8), one can set the bounds loose enough to include almost all ocean waveguides on Earth, but still reject much of the noise in $I_{2 \mathrm{DF}}\left(k_{r, \text { image }}, k_{f \text {,image }}\right)$. The $r$ in Eq. (3.9) should be set to the maximum range that one expects to see the source. All results presented in this chapter used Eqs. (3.8) and (3.9) with $r=5000$ $\mathrm{m}, c_{1}=1500 \mathrm{~m} / \mathrm{s}$, and $c_{2}=1800 \mathrm{~m} / \mathrm{s}$.

\section{Choosing The Window Size}

$I_{\text {win }}(r, f)$ is a rectangular window of $I(r, f)$, bounded by $\left(r_{\min }<r<r_{\max }\right)$ and $\left(f_{\min }<f<f_{\max }\right)$, inside of which the striation slope will be estimated using a 2D-DFT. The purpose of this subsubsection is to determine how one goes about choosing the window size. Denote the window size by

$$
\begin{aligned}
\Delta f & =f_{\max }-f_{\min } \\
\Delta r & =r_{\max }-r_{\min }
\end{aligned}
$$

To accurately determine the striation angle, the observation window of $I(r, f)$ must be large enough in $r$ and $f$ such that at least one full striation (from peak to trough to peak) is contained within the window, in each direction ( $r$ and $f$ ). The statement in the previous sentence can be quantified by noting that the frequency resolution of the DFT for some variable $x$ is $\Delta k_{x}=\frac{2 \pi}{\Delta x}$, so if the DFT of a signal is to distinguish the frequency of $k_{x}$ from the zero frequency, then one needs to 
observe at least $\Delta x=\frac{2 \pi}{k_{x}}$.

In theory one could use the $k_{r \text {,image,max }}$ derived Sec. 3.3.2 (originally derived in [18]) to determine the minimum $\Delta r$. However, only one term of the sum in Eq. (2.11) will lead to such a high value of $k_{r \text {,image }}$. So using $k_{r \text {,image,max }}$ would underestimate the minimum $\Delta r$ that is required to estimate the striation slope in practical situations. A better way to determine the minimum value of $\Delta r$ is to use the value of $k_{r, \text { image, } m l}$ averaged over $m$ and $l$.

To do this, first the approximate values of $\Delta k_{m l}(f)$ for an ideal waveguide will be calculated. Then the additional approximations required for non-ideal waveguides will be discussed. For an ideal waveguide, the difference in horizontal wavenumbers of modes not near cutoff can be written as [Eq. (2.22)]

$$
\Delta k_{m l}(f)=k_{m}(f)-k_{l}(f) \approx \frac{1}{2}\left(\frac{c}{2 \pi f}\right)\left(\frac{\pi}{d}\right)^{2}\left(m^{2}-l^{2}\right)
$$

Note that because the intensity is a sum of cosines and $\cos \left(\Delta k_{m l} r\right)=\cos \left(-\Delta k_{m l} r\right)$, only the absolute value of $\Delta k_{m l}$ is of interest. The average absolute value of the wavenumber differences can be determined by calculating the average value of $\left|m^{2}-l^{2}\right|:$

$$
\overline{\left(l^{2}-m^{2}\right)}=\frac{1}{M^{2}} \sum_{m=1}^{m=M} \sum_{l=1}^{l=M}\left|l^{2}-m^{2}\right|=\frac{M^{4}+M^{3}-M^{2}-M}{3 M^{2}}
$$

where $M$ is the number of propagating modes. The computer algebra system Mathematica was used to determine the formula for the sum. For $M \gg 1$,

$$
\overline{\left(l^{2}-m^{2}\right)} \approx \frac{1}{3} M^{2}
$$

For an ideal waveguide, Eq. (3.14) can be inserted into Eq. (3.12) to determine the approximate average horizontal wavenumber difference, and thus the average value of the image wavenumber $k_{r \text {,image }}$.

For non-ideal waveguides, this analysis assumes that the horizontal wavenumber differences are distributed similarly to that of an ideal waveguide, but are 
bounded by the maximum and minimum $k$ in the media:

$$
\Delta k_{m l}(f)=2 \pi f\left(\frac{1}{c_{\min }}-\frac{1}{c_{\max }}\right) \frac{l^{2}-m^{2}}{M^{2}}
$$

The mean horizontal wavenumber difference is then

$$
\overline{k_{r, \text { image }, m l}(f)}=\overline{\Delta k_{m l}(f)}=2 \pi f\left(\frac{1}{c_{\min }}-\frac{1}{c_{\max }}\right) \frac{1}{3}
$$

Conveniently, this does not depend on $M$.

For the average value of $k_{f \text {,image }}$, we use a similar argument. Begining with Eqs. (3.6) and (3.12):

$$
\overline{k_{f, \text { image }, m l}}=r \overline{\frac{\partial\left(\Delta k_{m l}(f)\right)}{\partial f}}=\left(\frac{r c \pi}{4 d^{2} f^{2}}\right) \overline{\left(m^{2}-l^{2}\right)}=\left(\frac{r c \pi}{4 d^{2} f^{2}}\right) \frac{M^{2}}{3}
$$

One can then insert $M$ for an ideal waveguide.

For non-ideal waveguides, this analysis assumes that the derivative of the horizontal wavenumbers with respect to $f$ are distributed similarly to that of an ideal waveguide (or equivalently, the group slownesses are distributed similarly to that of an ideal waveguide), bounded by the minimum and maximum group slownesses. In that case, the mean $k_{f, \text { image }, m l}$ is

$$
\overline{k_{f, \text { image }, m l}}=2 \pi r\left(\frac{1}{c_{\min }}-\frac{1}{c_{\max }}\right) \frac{1}{3}
$$

Eqs. (3.16) and (3.18) can be used to ensure that the window will contain one full striation of the average striation width. A larger window size could be used and could potentially lead to a more accurate striation slope estimate because the resolution of the 2D-DFT is inversely proportional to the window size. But because the slopes of the striations contained in $I(r, f)$ change with $r$ and $f$ according to Eq. (2.29), the window size should not be too large or it will contain striations with a wide range of slopes.

We now discuss how to choose the window size, given the trade-offs mentioned in the previous paragraph.

$I_{\text {win }}(r, f)$ will contain striation slopes ranging from $\frac{f_{\min }}{r_{\max }}$ to $\frac{f_{\max }}{r_{\min }}$. One way to 
choose the window size would be to make the range of striation slopes in the window equal the range of striation slopes represented by the 2D-DFT bin. According to Eq. (2.29), a window of size $\Delta r$ by $\Delta f$ centered at $r$ and $f$ with have slopes ranging from

$$
\frac{f+\Delta f / 2}{r-\Delta r / 2} \text { to } \frac{f-\Delta f / 2}{r+\Delta r / 2}
$$

A striation's slope is perpendicular to the angle (in $r, f$ space) of its cosine basis function, so an image wavenumber of $k_{r \text {,image }}$ and $k_{f \text {,image }}$ represents a striation with a slope of $-k_{r \text {,image }} / k_{f \text {,image }}$. Thus, an image wavenumber frequency bin of size $\Delta k_{r, \text { image }}$ by $\Delta k_{f, \text { image }}$ located at $k_{r \text {,image }}$ and $k_{f \text {,image }}$ represents striations ranging from

$$
-\frac{k_{r, \text { image }}+\Delta k_{r, \text { image }} / 2}{k_{f, \text { image }}-\Delta k_{f, \text { image }} / 2} \text { to }-\frac{k_{r, \text { image }}-\Delta k_{r, \text { image }} / 2}{k_{f, \text { image }}+\Delta k_{f, \text { image }} / 2}
$$

To make the range of striation slopes in the window equal the range of striation slopes represented by the 2D-DFT bin, one could in principle substitute $k_{r \text {,image }}=$ $\frac{2 \pi}{\Delta r}$ and $k_{f, \text { image }}=\frac{2 \pi}{\Delta f}$ into Eqs. (3.19) and (3.20), and then set the ranges of slopes equal to each other. The solution would depend on $k_{r \text {,image }}$ and $k_{f \text {,image }}$, for which one could use the average values derived in this section. The solution would also depend on $r$, for which one could choose some value in the middle of the search range. An exact analytic solution can be obtained and would ensure that the range of slopes represented by the 2D-DFT bin would equal the range of slopes in the window. However, doing so will only provide a relationship between $\Delta r$ and $\Delta f$, not values for both quantities, because there are an infinite number of combinations of $\Delta r$ and $\Delta f$ that could satisfy the equality.

A less quantitatively rigorous, but more pragmatic approach is used in the present work to determine the value of $\Delta r$ and $\Delta f$. We start with the heuristic that the window should be roughly three times the average striation width that we expect to see in each direction. This heuristic is motived by a desire for the 2DDFT bin representing the average image wavenumber expected to be a few 2D-DFT bin-widths away from both axes in Fig. 3.1(b). Thus we desire:

$$
\Delta r=\frac{3 \cdot 2 \pi}{\overline{k_{f, \text { image }, m l}}}, \quad \Delta f=\frac{3 \cdot 2 \pi}{\overline{k_{r, \text { image }, m l}(f)}}
$$


The $r$ in Eq. (3.18) should be set towards the lower end of the ranges over which one is searching for the source.

In the present work, $\Delta f$ was determined by using Eqs. (3.21) and (3.18) with $c_{1}=1500 \mathrm{~m} / \mathrm{s}, c_{2}=1800 \mathrm{~m} / \mathrm{s}, r=1000$ meters, resulting in $\Delta f \approx 81 \mathrm{~Hz}$. $\Delta r$ was determined by using Eqs. (3.21) and (3.16) with $c_{1}=1500 \mathrm{~m} / \mathrm{s}, c_{2}=$ $1800 \mathrm{~m} / \mathrm{s}$, and $f=525 \mathrm{~Hz}$ (the middle of the frequency range of the data presented in the next section), resulting in $\Delta r \approx 154$ meters.

The parameter values determined in this section $-k_{\mathrm{r}, \max }, k_{\mathrm{f}, \max }, \Delta f$ and $\Delta r$ - are used to process the simulated and both experimental data sets in this chapter. The parameter values were not "fine tuned" for each data set, illustrating the robustness of the method to the choice of the parameters. In fact, it was observed that all of the parameters can be adjusted by roughly a factor of 2 (increase or decrease) without drastically affecting the results for the data sets analyzed in the present chapter.

Equations (3.16), (3.18) and (3.21) provide practical estimates of the minimum source bandwidth and minimum horizontal aperture required for range estimation. These estimates are shown to be accurate with experimental data in Sec. 3.4.2. However, it is important to keep in mind that Eqs. (3.16), (3.18) and (3.21) were derived for range independent waveguides. Under some circumstances (e.g., a rough sea surface, or a source with a high temporal frequency $f$ ), effects such as incoherent scattering will smear out the high image-wavenumber striations, and a window larger than that predicted by Eq. (3.21) will be necessary to estimate a striation's slope. (See Sec. 7.3 for specific suggestions for future research)

\subsection{Results}

\subsubsection{D-DFT technique applied to simulated data}

In this subsection, the striation angle finding technique based on the 2D-DFT is tested on the simulated acoustic intensity shown in Fig. 3.3, which is from the same Pekeris waveguide used to produce Fig. 2.3. After finding the striation angle, Eq. (3.1) is used to estimated the range to the acoustic source, assuming $\beta=1$.

First, $I(r, f)$ is divided into several windowed segments, $I_{\text {win }}(r, f)$, spread on a grid throughout the $(r, f)$ plane. The striation angle is then estimated in each 


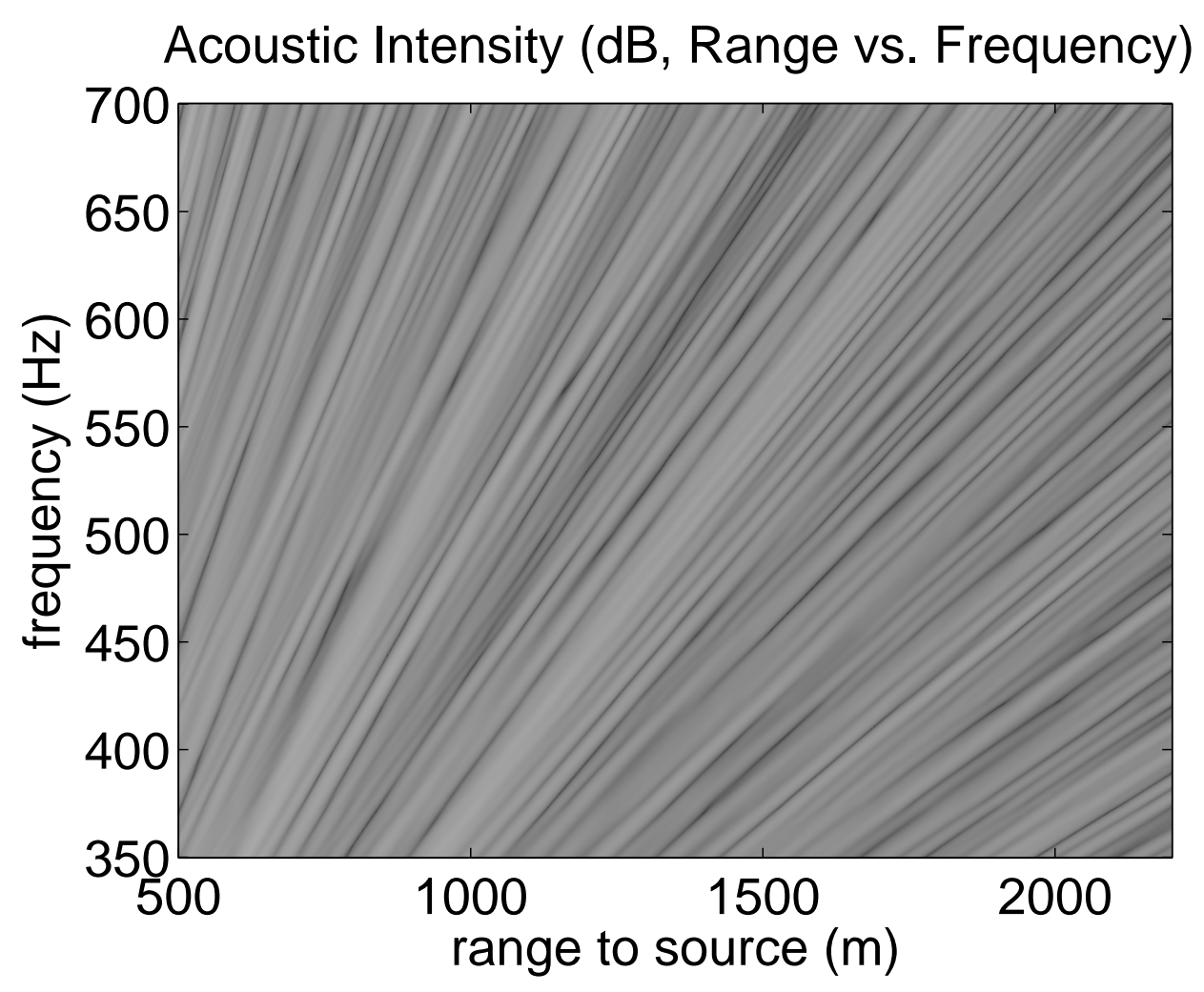

Figure 3.3: Acoustic intensity ( $\mathrm{dB}$, arbitrary reference) in a Pekeris waveguide plotted versus range and frequency, $I(r, f)$, for a receiver depth of 20 meters and a source depth of 40 meters. 
$I_{\text {win }}(r, f)$ using the technique described in the previous section.

The resulting slope estimates are shown in Fig. 3.4(a). Each slope estimate is associated with a range estimate, which are shown in Fig. 3.4(b).

In order to use all of the data, one needs to ensure that every pixel in Fig. 3.3 is contained inside at least one window. In fact, one can let the windows overlap. In the present analysis, the windows overlapped by about 50\% in both the $r$ and the $f$ directions.

One can average each column of Fig. 3.4(b) to obtain more accurate range estimates. The result of such averaging, plotted versus the true range to the source, is shown in Fig. 3.4(c).

The estimates are biased by about $10 \%$. This is most likely do to the fact that the derivation of the waveguide invariant does not take into account the $\frac{1}{r}$ cylindrical spreading, which will cause the actual slopes to be steeper than that predicted by the waveguide invariant. However it could also be due to the other approximations made when the waveguide invariant, such as the dependence of the mode shapes on frequency. Because this error is less than the expected range estimate accuracy for experimental data, it will not be addressed further in the present analysis.

\subsubsection{D-DFT technique applied to experimental data}

The experimental data presented in this chapter was collected during GLINT08, an experiment performed during the summer of 2008 near Pianosa Island, Italy. Two sound speed profiles measured about an hour before and an hour after the acoustic data were collected are shown in Fig. 3.5(a), but note that this information was not used by the range estimation algorithm.

An acoustic source was lowered $40 \mathrm{~m}$ below the ocean surface from the R/V Leonardo, which was using dynamic positioning to keep its position as fixed as possible. Due to a malfunctioning GPS unit, the acoustic source's position had an uncertainty of $100 \mathrm{~m}$. The signal projected from the source was pseudo-random white noise with a with an approximately flat spectrum from 300 to $750 \mathrm{~Hz}$ and a frequency-integrated source level of $150 \mathrm{~dB}$ re: $1 \mu \mathrm{Pa}$ at $1 \mathrm{~m}$. Due to equipment limitations, the signal was a single 65000 point realization of white noise that was repeated back-to-back every 5.33 seconds.

An acoustic receiver was towed directly toward the acoustic source from a range 
(a) Acoustic Intensity (dB, Range vs. Frequency)

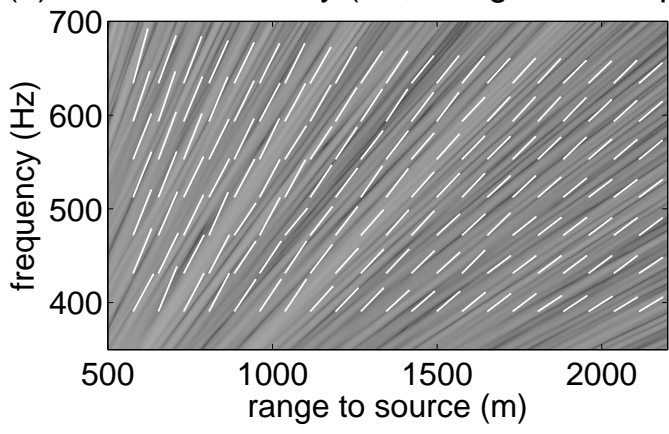

(b) Ranges Estimates (m)

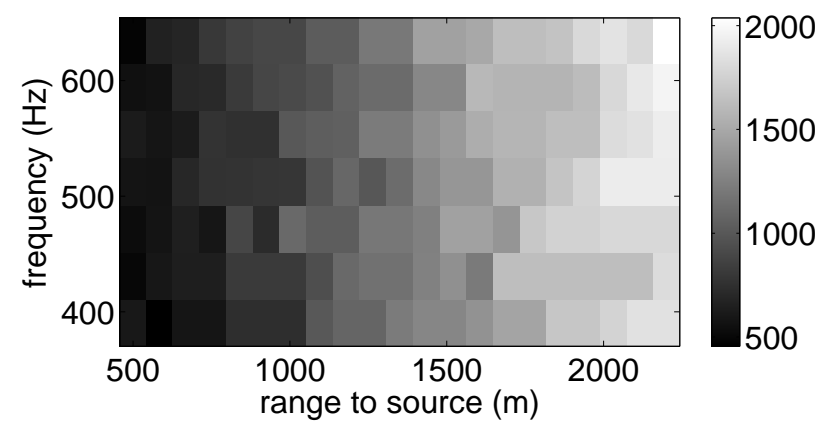

(c) Estimated Ranges vs. True Ranges

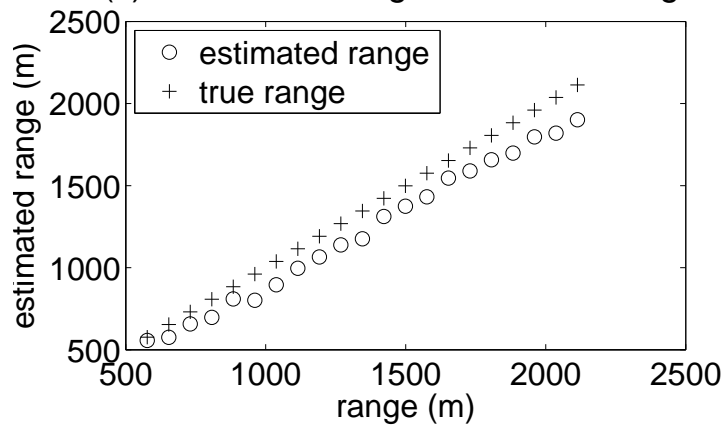

Figure 3.4: Range estimates for a simulated Pekeris waveguide. (a) Same as Fig. 3.3, but with the estimated slopes superimposed as white solid lines. (b) Range estimates based on each slope in part (a) plotted versus the true range. (c) Range estimates versus true range, obtained by averaging each column of part (b). The bias is discussed Sec. 3.4.1. 


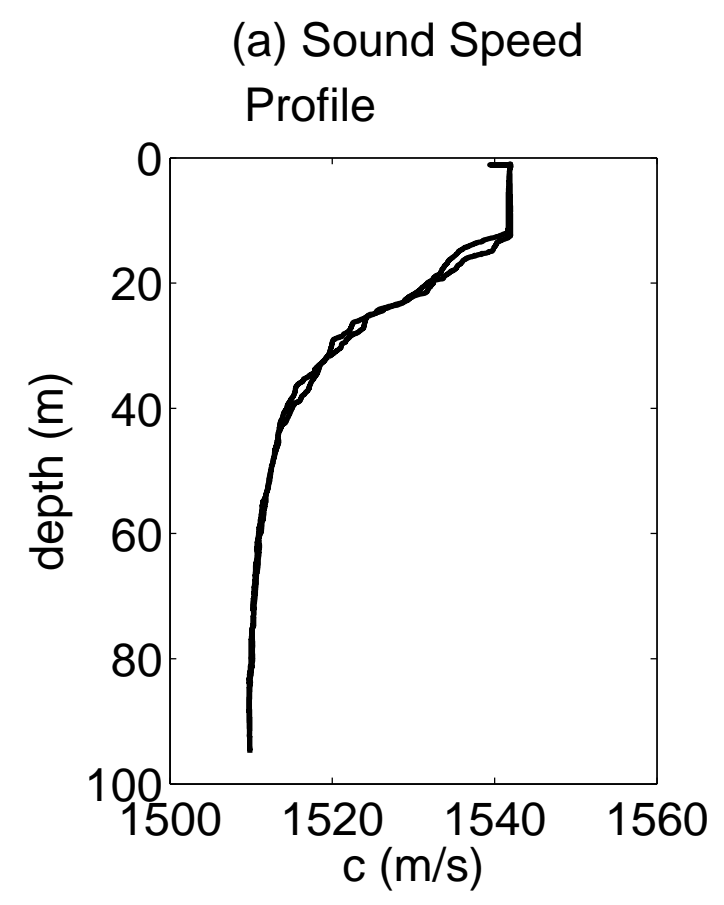

(b) Sound Speed

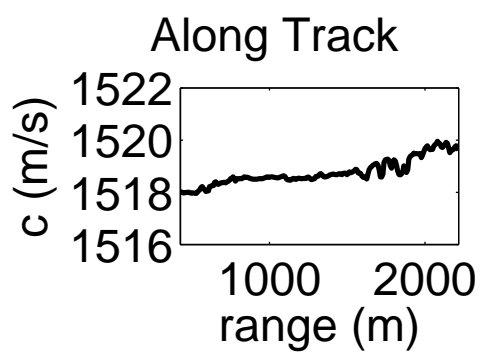

(c) Bathymetry and Hydrophone Depth

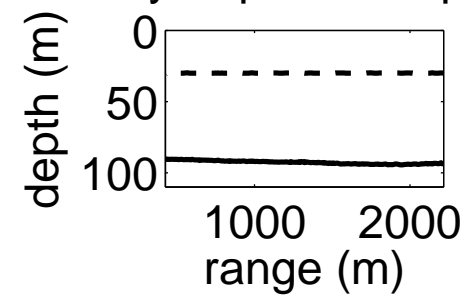

Figure 3.5: (a) Sound speed profiles taken before and after the acoustic data collection. (b) Sound speed at the hydrophone's depth plotted versus range along the track. (c) Water column depth (solid line) and hydrophone depth (dashed line) plotted versus range from the source along the hydrophone's path. 
of 2,200 meters to about 500 meters at a speed of $1.5 \mathrm{~m} / \mathrm{s}$ and at a depth of 30 meters. This segment of the experiment is referred to as the incoming segment. The acoustic receiver was then lowered to 50 meters, and towed away from the source at $1.5 \mathrm{~m} / \mathrm{s}$ back out to a range of 2,200 meters. This segment is referred to as the outgoing segment. The acoustic receiver was moving continuously, so the all of the presented data was collected in less than 1 hour. There was a small Doppler shift of about $1 \%$ due to receiver motion. Such a shift may cause range estimate errors of about $1 \%$, which is negligible compared to the overall expected accuracy and thus will be ignored. The acoustic receiver location had an uncertainty of 50 meters.

The acoustic data were sampled at a frequency of $4 \mathrm{kHz}$. The window length used to estimate the spectrum of the received signal had to be 21,334 points long so that it corresponded to the 5.33 second repetition rate of the signal. Under other circumstances, one would be free to choose other window lengths that do not strongly depend upon the signal characteristics. Because the acoustic receiver was moving at an approximately $1.5 \mathrm{~m} / \mathrm{s}$, each spectrum (each column of Fig. 3.6) represents $\approx 5.33 \mathrm{~s} \times 1.5 \mathrm{~m} / \mathrm{s}=8$ meters in distance that the acoustic receiver traveled. The Blackman-Tukey method of spectrum estimation was used to estimate each spectrum (each column of Fig. 3.6). Because the acoustic receiver was towed at an approximately constant rate, the spectrogram of the recorded time series is $I(r, f)$.

Once the spectrogram, $I(r, f)$, was calculated, the processing method was exactly the same as that used for the simulated data. The same parameter values were used.

\section{Incoming segment}

The water column depth, acoustic receiver's depth and sound speed along the hydrophone's path are plotted in Fig. 3.5(b) and (c). The measured acoustic field from $0 \mathrm{~Hz}$ to $2 \mathrm{kHz}$ is shown in Fig. 3.6. The results of the range estimation algorithm are shown in the same format as the simulated results, in Figs. 3.7(b) and 3.7(c).

There is extremely good qualitative agreement between the angle determined by the striation angle finding algorithm and the striation angle as it appears to a human observer, as can be seen in Fig. 3.7(a). At source ranges larger than 1000 meters, the estimated range tends to be less than the true range. This could be the 
result of any number of effects, including: 3-d propagation effects, range inhomogeneities, the temporal stationarity of the SSP while the field was being measured, the approximations used when deriving the waveguide invariant. Sec. 3.4.3 shows a simulated spectrogram for comparison.

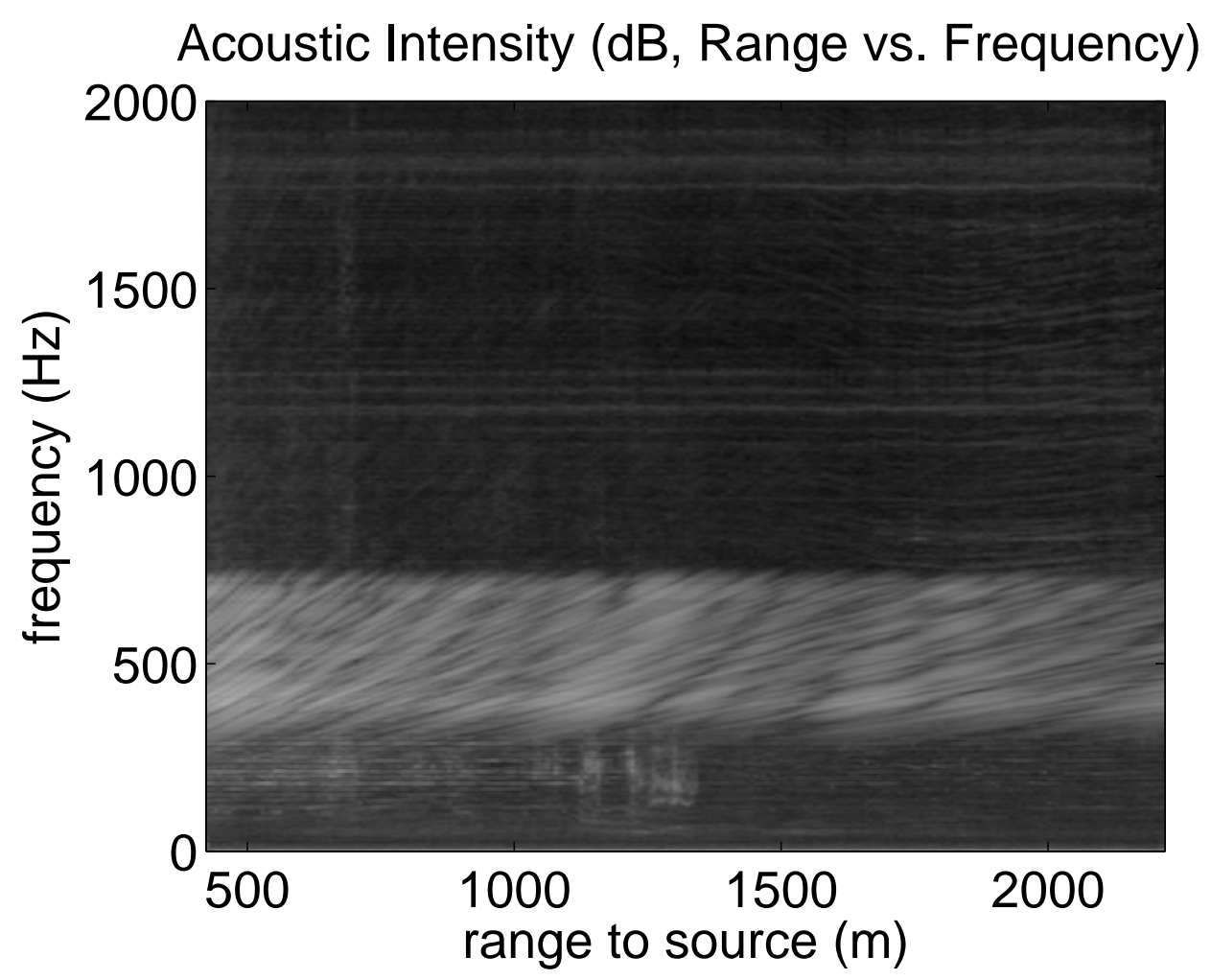

Figure 3.6: The acoustic intensity ( $\mathrm{dB}$, arbitrary reference) as measured by the acoustic receiver for the incoming segment of the experiment. The striations are clearly visible in the frequency range of the source, from 350 to $700 \mathrm{~Hz}$.

\section{Outgoing Segment}

The watercolumn depth and sound speed along the hydrophone's path were similar to the incoming segment (see Fig. 3.5). The measured acoustic field, from 0 to $2 \mathrm{kHz}$ is shown in Fig. 3.8. There is a loud interfering broadband acoustic source during the portion of the spectrogram corresponding to a range of 1000 to 2000 
(a) Acoustic Intensity (dB, Range vs. Frequency)

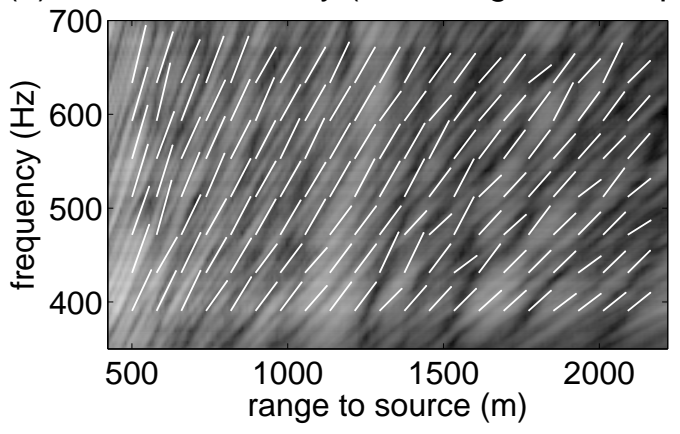

(b) Ranges Estimates (m)

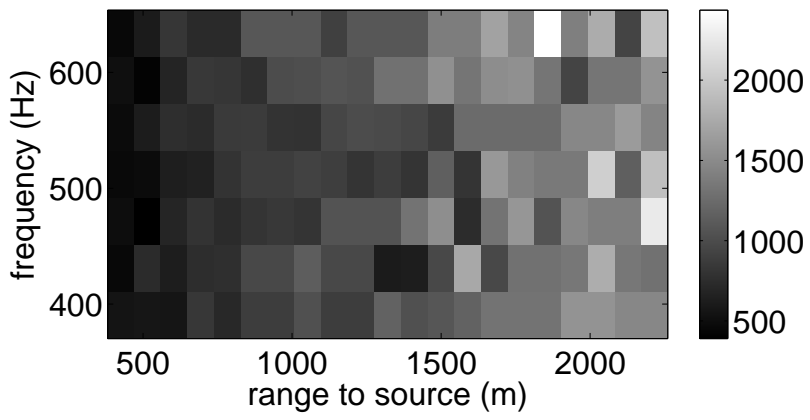

(c) Estimated Ranges vs. True Ranges

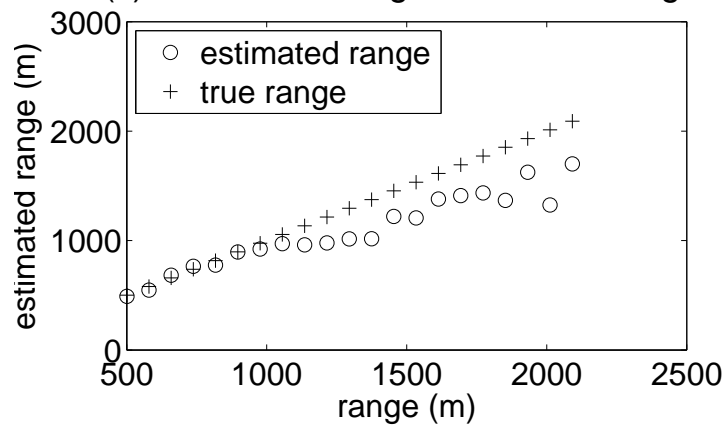

Figure 3.7: Range estimates for the incoming segment of the experiment. (a) A zoom-in of Fig. 3.6 on the frequencies of interest, with the estimated slopes superimposed as solid white lines. (b) Range estimates based on each estimated slope in part (a), plotted versus the true range. (c) Range estimates versus true ranges, obtained by averaging each column of part (b). 
meters. As one can see in Fig. 3.9, this does effect the estimates, but not as much as one might expect. The estimates are only adversely affected at ranges of 1400 to 1800 meters, when the striations in the spectrogram from the interferer are nearly parallel to the striations that are expected from the experimental acoustic source. If one did not know a priori that the acoustic receiver was moving away from the acoustic source of interest, then the estimates may have been affected more adversely because one would have had to search over the full 180 degrees. The range estimates are accurate within a few hundred meters, excluding the estimates when the true range was 1400 to 1800 meters.

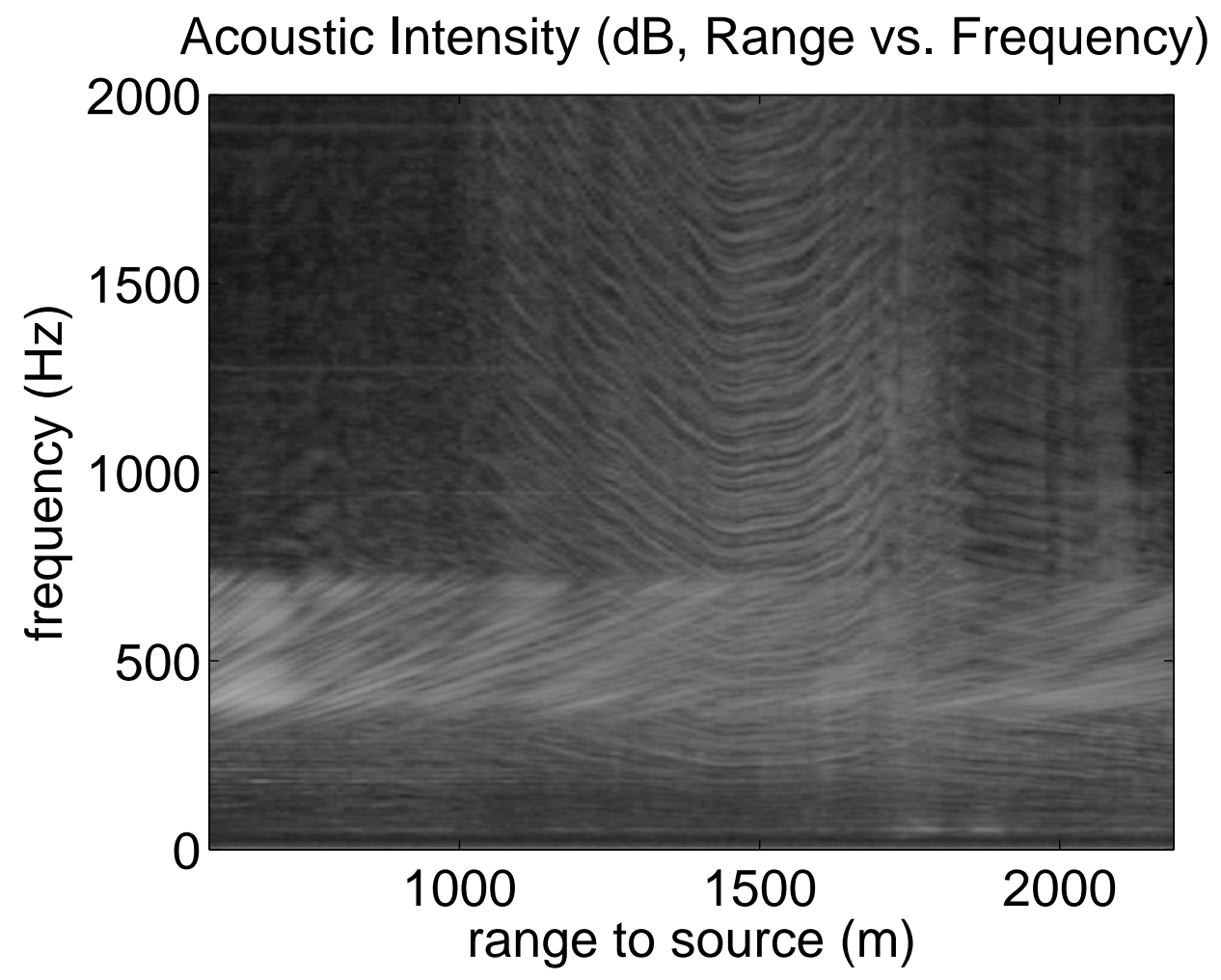

Figure 3.8: The acoustic intensity ( $\mathrm{dB}$, arbitrary reference) as measured by the acoustic receiver along its track away from the source. Note the striations in the frequencies of the acoustic source $(350$ to $700 \mathrm{~Hz}$ ) and the interfering source present during times corresponding to ranges from 1000 to 2000 meters. 
(a) Acoustic Intensity (dB, Range vs. Frequency)

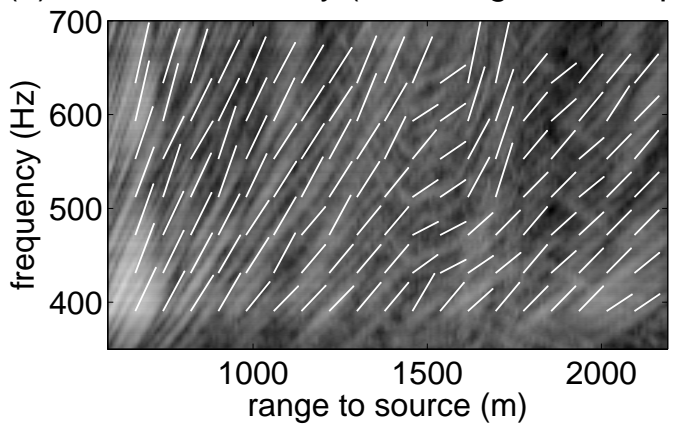

(b) Ranges Estimates (m)

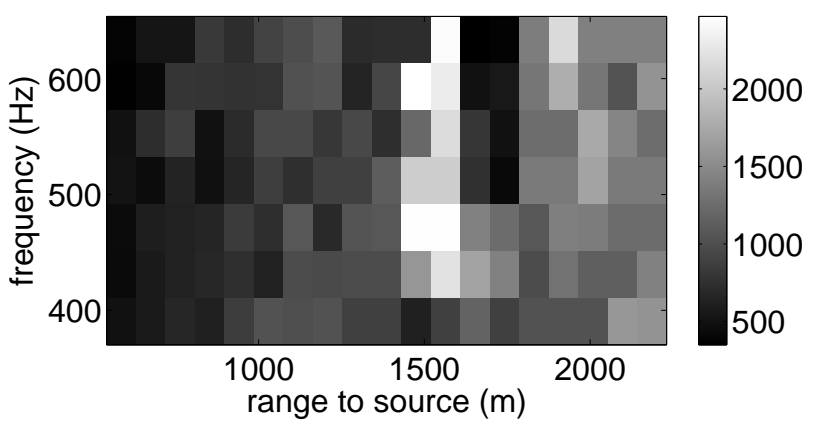

(c) Estimated Ranges vs. True Ranges

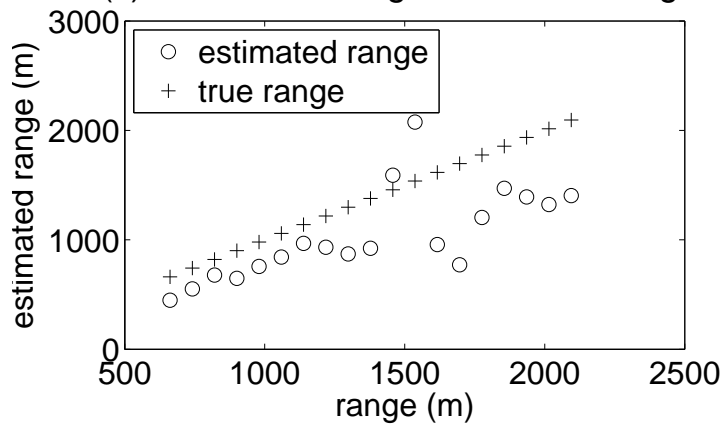

Figure 3.9: Range estimates for the outgoing segment of the experiment. (a) A zoom-in of Fig. 3.8 on the frequencies of interest, with the estimated slopes superimposed as solid white lines. (b) Range estimates based on each estimated slope in part (a), plotted versus the true range. (c) Range estimates versus true range, obtained by averaging each column of part (b). Large errors occur when the interfering source was present. 
So far, the present chapter has dealt exclusively with the signal recorded from a single hydrophone. However, data was collected from a 32-element horizontal array of hydrophones. Chapter 5 discusses how to process the data from all the hydrophones in order to reduce the noise in Fig. 3.8. The result of such filtering can be seen in Fig. 5.4(b). One can then apply the passive ranging algorithm to the array-filtered data in Fig. 5.4(b); the result is shown in Fig. 3.10. Note that Figs. 3.8 and 3.10 are from the experimental data set; the difference is that Fig. 3.8 used data from a single hydrophone of the array whereas Fig. 3.10 used data all of the hydrophones of the array. 
(a) Acoustic Intensity (dB, Range vs. Frequency)

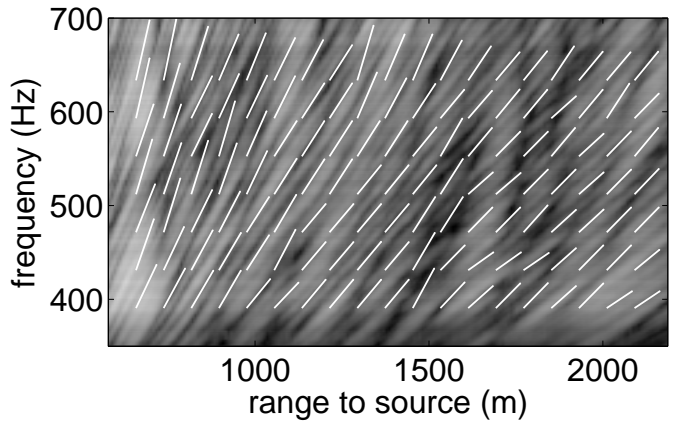

(b) Ranges Estimates (m)

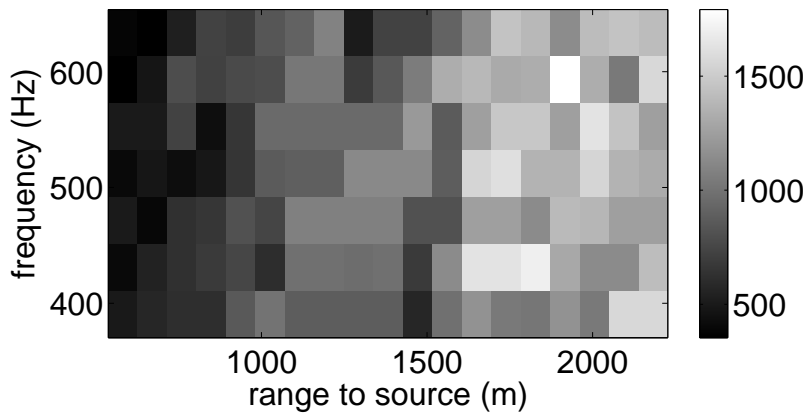

(c) Estimated Ranges vs. True Ranges

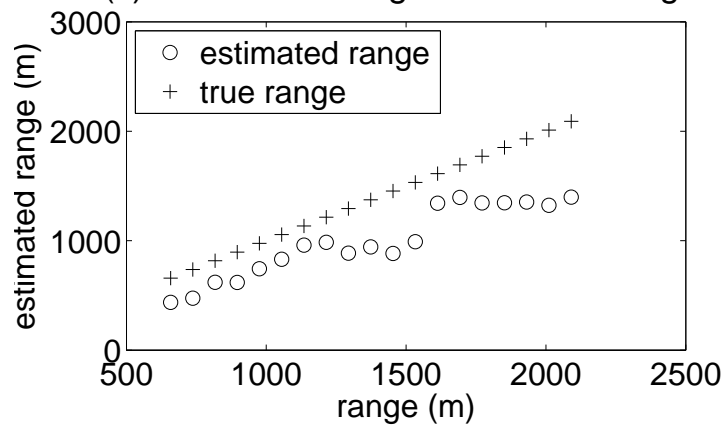

Figure 3.10: Range estimates for the outgoing segment of the experiment using array-filtered data. This figure is similar to Fig. 3.9, but data from the 32-element array has been used to remove the noise from sources besides the source of interest. (a) A zoom-in of Fig. 5.4 on the frequencies of interest, with the estimated slopes superimposed as solid white lines. (b) Range estimates based on each estimated slope in part (a), plotted versus the true range. (c) Range estimates versus true range, obtained by averaging each column of part (b). 


\subsubsection{Validity of $\beta=1$ assumption}

Section 3.2 discussed that although usually $\beta \approx 1$ in shallow water environments, that may not always be the case (see Sec. 2.3.3 or [51]). Determining when one can assume that $\beta \approx 1$ is still an active area of research, but some guidelines are given in $[51,49]$, and other papers on the waveguide invariant. Chapter 4 presents some original research on this topic.

All of the analysis in the present chapter assumed $\beta=1$, so it is worthwhile to simulate the acoustic field in an environment similar to the environment where the experimental data was collected, in order to determine if $\beta$ differs significantly from the assumed value. (We expect it not to, otherwise we wouldn't have been able to accurately estimate the range to the source in the previous section.)

To do this, the normal mode program Kraken [48] was used. The sound speed profile used was that collected during the experiment [shown in Fig. 3.5(a)]. The source and receiver geometry were the same as the incoming portion of the experiment. Because the bottom bathymetry and the sound speed profile changed very little with range, the environment was modeled as being range independent. The bottom properties were unknown so typical values for a bottom half-space were used $\left(c_{\text {bottom }}=1650 \mathrm{~m} / \mathrm{s}, \rho_{\text {bottom }}=1.5 \mathrm{~g} / \mathrm{cm}^{3}, \alpha=0.5 \mathrm{~dB} / \lambda\right)$.

Figure 3.11 shows the simulated spectrogram. The white lines have slopes corresponding to $\beta=1$. It can be seen that there are some striations with slopes that are slightly steeper than the $\beta=1$ lines, but almost no striations have slopes that are shallower than the $\beta=1$ lines. This could explain why the ranges tended to be underestimated in some parts of the incoming segment of the experiment [Fig. 3.7(c)], and suggests that $\beta$ may have a value slightly larger than one for this particular environment and source-receiver geometry.

The fact that the range estimates from the experimental data in Figs. 3.7(c) and $3.10(\mathrm{c})$ were between about $75 \%$ to $100 \%$ of the true range implies that $\beta$ had a value between about 1 and $4 / 3$ for the environment where the experimental data was collected. Later in this thesis, a method is developed for calculating the value of $\beta$. Section 4.5.4 shows that $\beta$ changes with horizontal wavenumber, and that it has a value of slightly less than one for this particular environment (for the SRBR modes). Thus there is a discrepancy between the predicted value of $\beta$ being slightly less than one, and the measured value of $\beta$ being slightly greater than 
one ([49] and [32] also have experimental examples of $\beta$ being slightly greater than one). This discrepancy might be due to the non-SRBR modes which have different $\beta$ values, the $1 / \sqrt{r}$ spreading, or attenuation (imaginary components of the horizontal wavenumbers). [51] provides some explanation for this discrepancy, but there is certainly room for further research.

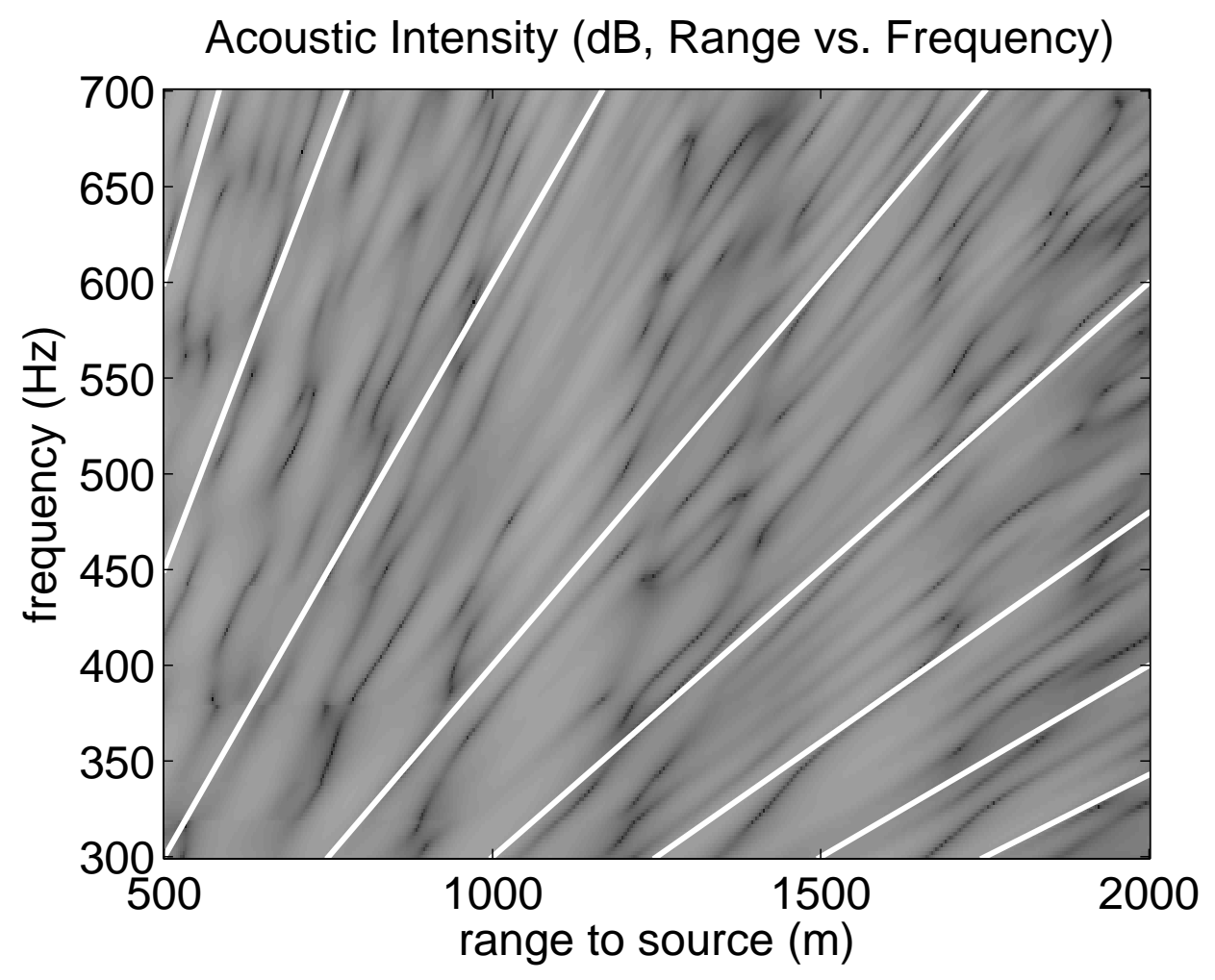

Figure 3.11: A simulated spectrogram for the environment used to collect the experimental data. The white lines correspond to $\beta=1$. Most of the striations correspond to $\beta \approx 1$, with a few exceptions.

\subsection{Chapter summary}

A processing scheme based on the waveguide invariant and the 2D-DFT of $I(r, f)$ was used to estimate an acoustic source's range using simulated data and two sets of experimental data. The processing techniques used did not require human inter- 
pretation of any images in order to obtain the range estimate, making the techniques suitable for implementation on an autonomous platform.

A relationship between the average image-wavenumbers in the 2D-DFT of $I(r, f)$ and the acoustic waveguide parameters was used to determine the minimum observation window size of $I(r, f)$ required for range estimation. A similar relationship was used to reject noise in $I(r, f)$.

The same set of signal processing parameter values (maximum image wavenumber, and window size in range and frequency) was used for both simulated and experimental data, showing that the signal processing parameters' values do not need to be fine-tuned for each data set. The range estimates were based on the assumption (approximation) that $\beta$ is a single scalar value, and is equal to one. The range estimates were accurate to within about $25 \%$ despite using only a minimal amount of a priori knowledge about the environment. The technique appeared to be robust, but more experimental data would be needed to determine the robustness of these algorithms in other environmental conditions. 


\section{Chapter 4}

\section{A WKB modal approach to calculating the waveguide invariant}

The previous chapter illustrated that the waveguide invariant is a powerful concept for explaining range-frequency striations in experimental data. It is somewhat surprising that the strong non-uniformities present in experimental SSP in the previous chapter did not have a large effect on the value of $\beta$ observed in $I(r, f)$. The present chapter develops a method for calculating the waveguide invariant that illustrates the relationship between the SSP and $\beta$, which provides some insight into why $\beta \approx 1$ in most shallow-water waveguides. ${ }^{1}$

In this chapter, $m$ and $l$ refer to the mode numbers just as they did in previous chapters. This chapter also uses $n$ to refer to the mode number, but $n$ is used only when the mode number comes from the WKB approximation. The reason for this distinction will become clear later.

\footnotetext{
${ }^{1}$ This chapter is based on "A modal Wentzel-Kramers-Brillouin approach to calculating the waveguide invariant for non-ideal waveguides" by Kevin L. Cockrell and Henrik Schmidt [Under review for publication in The Journal of the Acoustical Society of America]
} 


\subsection{Introduction}

In Chuprov's original paper on the waveguide invariant, he defines $\beta$ in two different ways. The first way uses normal modes to describe the acoustic field, and the second way uses ray theory to describe the acoustic field [18]. In general, normal modes is accurate under a wider range of circumstances than ray theory, however, normal modes tends to be less amenable to non-numerical analysis than ray theory.

Previous research on the waveguide invariant using ray theory has used both numerical techniques and analytic techniques [18, 24, 22], with the analytic techniques providing some insight into how the SSP affects $\beta$.

Previous research on the waveguide invariant using normal modes has mostly used numerical techniques to calculate $\beta[18,51,21,52]$, which has provided many useful insights but is not conducive for understanding how the SSP affects $\beta$.

This chapter investigates the waveguide invariant using a the WKB approximation to normal modes, which can be thought of as "half way" between full-field normal modes and geometric ray theory. The modal WKB description is accurate under a wider range of circumstances than geometric ray theory but is more amenable to (non-numerical) analysis than full-field normal modes.

$\beta$ can be defined directly in terms of the normal modes' horizontal wavenumbers, as is shown in Eq. (2.15). To make the dependence of $\beta$ on the modes numbers $m$ and $l$ and the frequency $\omega$ more explicit, we write Eq. (2.15) as:

$$
\beta(m, l, \omega)=-\frac{1}{\omega} \cdot \frac{k_{r}(m, \omega)-k_{r}(l, \omega)}{\frac{\partial\left(k_{r}(m, \omega)-k_{r}(l, \omega)\right)}{\partial \omega}}
$$

where $k_{r}(i, \omega)$ is the horizontal wavenumber of mode $i$ at frequency $\omega$.

In Sec. 2.3.2 it was shown analytically that $\beta \approx 1$ for ideal waveguides. References [18] and [11] analytically show that $\beta \approx-3$ for surface-trapped modes in waveguides with $n^{2}$-linear sound speed profiles (SSP). As discussed in Sec. 2.3, experimental data and numerical simulations have shown that many realistic shallowwater waveguides can be well approximated by an ideal waveguide for the purpose

of calculating $\beta$ (i.e., $\beta \approx 1$ for many shallow-water waveguides). However, the 
analysis in Sec. 2.3 (originally from [18], [11, Sec. 6.7.2], and [34, Sec. 2.4.6]) does not lend itself to understanding why $\beta$ is not strongly affected by the nonuniformities present in a realistic SSPs, as opposed to the pressure field itself which is strongly affected (two examples are shown in Fig. 2.4). In fact, the derivation in Sec. 2.3.2 showing that $\beta \approx 1$ for an ideal waveguide only considers modes far from cutoff (i.e., low-order modes at high frequency). Paradoxically, those are precisely the modes whose $k_{r}$ values will be most affected by the non-uniformities in the SSP ${ }^{2}$; those modes will "tuck" into the local sound speed minimums and will not behave like modes in an ideal waveguide.

This chapter develops a method for calculating $\beta$ based on the WKB approximation, which is an approximate method for solving for the horizontal wavenumbers in a waveguide. The relationship between the WKB approximation and $\beta$ is briefly mentioned in the original waveguide invariant paper [18]. Later, Brown et al. used the WKB approximation to show the relationship between between $\beta$ and the ray stability parameter $\alpha[14,6]$. The present chapter investigates how nonuniformities in a SSP will affect $\beta$. The resulting method is then applied to a few canonical waveguides and compared with the values of $\beta$ calculated numerically from the normal mode program Kraken [48].

The main results are:

- If the WKB approximation is used, the derivatives required to calculate $\beta$ can be performed implicitly, circumventing the need to obtain explicit solutions for $k_{r}(m, \omega)$ and allowing for the inclusion of a fluid bottom halfspace. The resulting estimate of $\beta$ is then an explicit function of the horizontal wavenumber instead of being an explicit function of the mode number.

- When the bottom halfspace is modeled as a vacuum, the value of $\beta$ for all horizontal wavenumbers and all frequencies is a function of a single parameter: the phase slowness $S_{p}$.

- The value of $\beta$ can be directly related to two one-dimensional curves: the depth-integrated vertical wavenumber as a function of phase slowness and the seafloor reflection coefficient as a function of phase slowness. These

\footnotetext{
${ }^{2}$ Chuprov mentions this fact later in his paper when discussing the waveguide invariant in deepwater waveguides.
} 
curves show that $\beta$ for surface-reflected bottom-reflected (SRBR) modes is not strongly affected by the details of the SSP, but that $\beta$ for non-SRBR modes is strongly affected by the details of the SSP.

- A qualitative argument suggests that $\beta \approx 1$ for SRBR modes in waveguides where the seafloor is modeled as a vacuum, regardless of the SSP. $\beta$ can take on a wide range of values for non-SRBR modes.

\subsection{Calculating $\beta$ using the WKB approximation}

To calculate $\beta$, the WKB approximation is used to determine the modal horizontal wavenumbers. Details of the WKB approximation as it applied to calculating the modal horizontal wavenumbers can be found in [11, Sec. 6.7], [33, Sec. 2.5], [5], and [60, Sec. 2.9] (See Appendix B for even more references). All results in this chapter apply only to propagating modes with no more than 2 turning points (depths

where $k_{z}\left(z, k_{r}\right)=0$. see Sec. 2.2.2), and to waveguides with bottoms that are either lossless homogeneous fluid halfspaces or vacuums. Because the quantity of interest in the present analysis is the acoustic intensity, we note that the WKB approximation is usually more accurate at calculating the acoustic intensity than it is at calculating the complex acoustic field. As discussed in [8, Sec. 48.5], this is because the error of the WKB-approximated acoustic intensity depends on the errors of the differences of the horizontal wavenumbers, as opposed to the error of the WKB-approximated complex acoustic field which depends on the errors of the value of the individual horizontal wavenumbers.

Under the WKB approximation, the horizontal wavenumbers are calculated by solving for $k_{r}$ in the equation [5]

$$
n=\phi\left(k_{r}, \omega\right)+\Delta \phi_{\mathrm{dn}}\left(k_{r}, \omega\right)+\Delta \phi_{\mathrm{up}}\left(k_{r}, \omega\right)+1
$$


where $n$ is the mode number $(1,2,3, \ldots)$,

$$
\begin{aligned}
\phi\left(k_{r}, \omega\right) & =\frac{1}{\pi} \int_{z_{1}}^{z_{2}} k_{z}\left(z, k_{r}\right) d z \\
& =\frac{1}{\pi} \int_{z_{1}}^{z_{2}}\left(\sqrt{\left(\frac{\omega}{c(z)}\right)^{2}-k_{r}^{2}}\right) d z
\end{aligned}
$$

where $z_{1}$ and $z_{2}$ are either the sea surface $(z=0)$ and sea floor $(z=d)$ respectively, or a $z$ at which $k_{z}(z)=0$ (a turning point). And with:

$$
\Delta \phi_{\text {up }}\left(k_{r}, \omega\right)=\left\{\begin{array}{ll}
-\frac{1}{2} & \text { if } k_{r}<k(0) \quad \text { (i.e., mode reflects from surface) } \\
-\frac{1}{4} & \text { if } k_{r}>k(0)
\end{array} \quad\right. \text { (i.e., mode has an upper turning point) }
$$

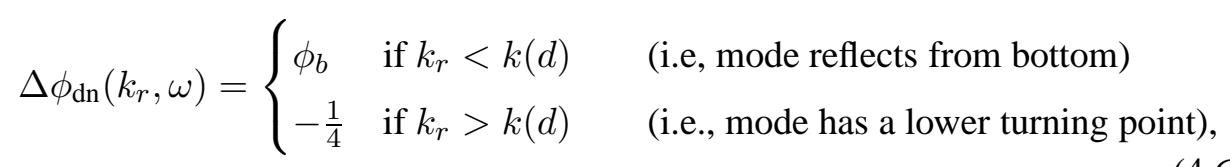

where $\phi_{b}\left(k_{r}, \omega\right)$ is $\frac{1}{2 \pi}$ times the phase angle of the bottom half-space reflection coefficient. $\left(-1 / 2 \leq \phi_{b}\left(k_{r}, \omega\right) \leq 0\right)$ for a fluid bottom and $\phi_{b}\left(k_{r}, \omega\right)=-1 / 2$ for a vacuum bottom. Figure 4.1 discusses how to interpret Eq. (4.3) (See Fig. 2.1 for a definition of SRBR modes). 
(a)

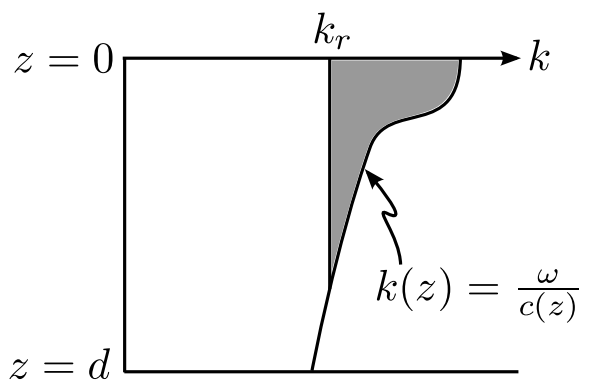

(b)

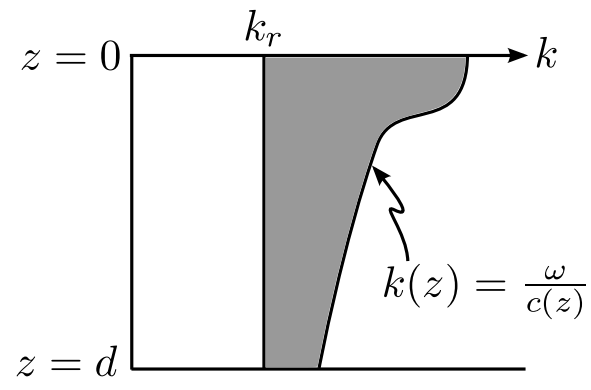

Figure 4.1: Illustration of the WKB approximation applied to calculating the horizontal wavenumbers $k_{r}$. The shaded area is related to (but not equal to) $\int k_{z}\left(z, k_{r}\right) d z$, which is used to determine the values of $k_{r}$ that correspond to propagating modes. Subfigure (a) demonstrates that when $k_{r}$ is greater than the minimum $k(z)$ in the water column (which corresponds to non-SRBR modes), the shape of $\int k_{z}\left(z, k_{r}\right) d z$ as a function of $k_{r}$ will depend strongly on the detailed shape of the the SSP - especially the part of the SSP near $k(z)=k_{r}$. Subfigure (b) demonstrates that when $k_{r}$ is less than the minimum $k(z)$ in the water column (which corresponds to SRBR modes), the shape of $\int k_{z}\left(z, k_{r}\right) d z$ as a function of $k_{r}$ does not depend strongly on the details of the SSP because the integral tends to average out any "roughness" in the SSP. A similar argument can be made regarding the dependence of $\int k_{z}(z) d z$ on $\omega$. 
Eq. (2.15) can be manipulated to facilitate a geometric interpretation of $\beta$ in terms of the WKB approximation. The numerator and denominator of Eq. (2.15) can be divided by an arbitrary value without affecting the value of the fraction. Specifically, one can divide the numerator and denominator by the difference in the mode numbers $(m-l)$ so that

$$
\beta(m, l, \omega)=-\frac{1}{\omega} \cdot \frac{\left(k_{r}(m, \omega)-k_{r}(l, \omega)\right) /(m-l)}{\frac{\partial\left(k_{r}(m, \omega)-k_{r}(l, \omega)\right)}{\partial \omega} /(m-l)} .
$$

A line connecting the points $\left(k_{r}(m, \omega), m\right)$ and $\left(k_{r}(l, \omega), l\right)$, as shown in Fig. 4.2 with $m=2$ and $l=4$, has a slope of $(m-l) /\left(k_{r}(m, \omega)-k_{r}(l, \omega)\right)$. This allows one to interpret the numerator of Eq. (4.7) as the reciprocal of that slope.

(a)

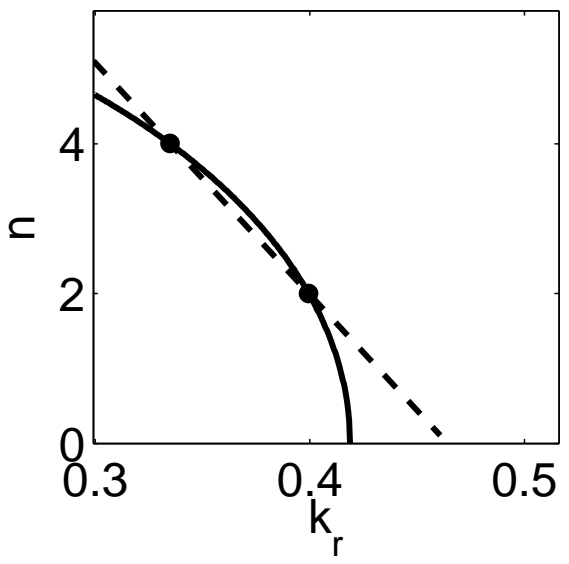

(b)

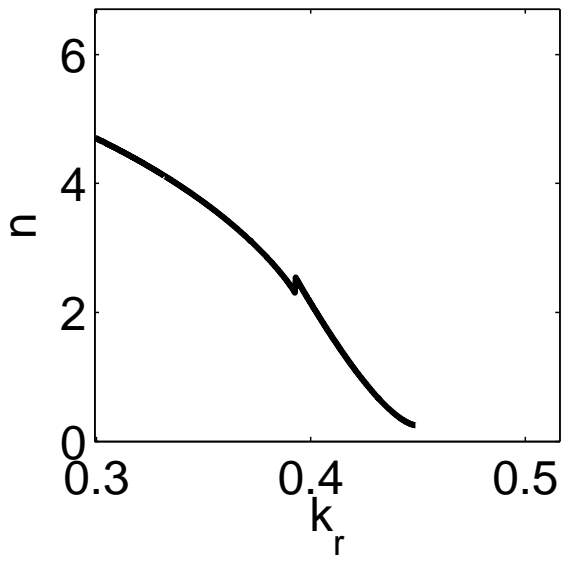

Figure 4.2: (a) A plot of $n$ versus $k_{r}$ for an ideal waveguide using the WKB approximation $(f=100 \mathrm{~Hz}, d=50 \mathrm{~m}, c=1500 \mathrm{~m} / \mathrm{s})$. The two points plotted correspond to modes 2 and 4 . The reciprocal of the slope of the line connecting the two points can be used to facilitate interpretation of the waveguide invariant $\beta$, as described in Sec. 4.2. (b) A plot of $n$ versus $k_{r}$ for a waveguide with an $n^{2}$-linear sound speed profile and pressure-release boundary conditions $(f=100 \mathrm{~Hz}, d=50 \mathrm{~m}, c(0)=$ $1400 \mathrm{~m} / \mathrm{s}, c(d)=1600 \mathrm{~m} / \mathrm{s})$. The discontinuity at $k_{r}=\omega / c(d)$ is due to the WKB approximation ( $\phi_{\mathrm{dn}}$ as defined in Eq. (4.6)).

$\beta(m, l, \omega)$ depends on how $\left(k_{r}(m, \omega)-k_{r}(l, \omega)\right)$ depends on frequency. One can visualize that dependence by plotting $n$ as a function of $k_{r}$ and of $\omega$, as shown 
for an ideal waveguide in Fig. 4.3.

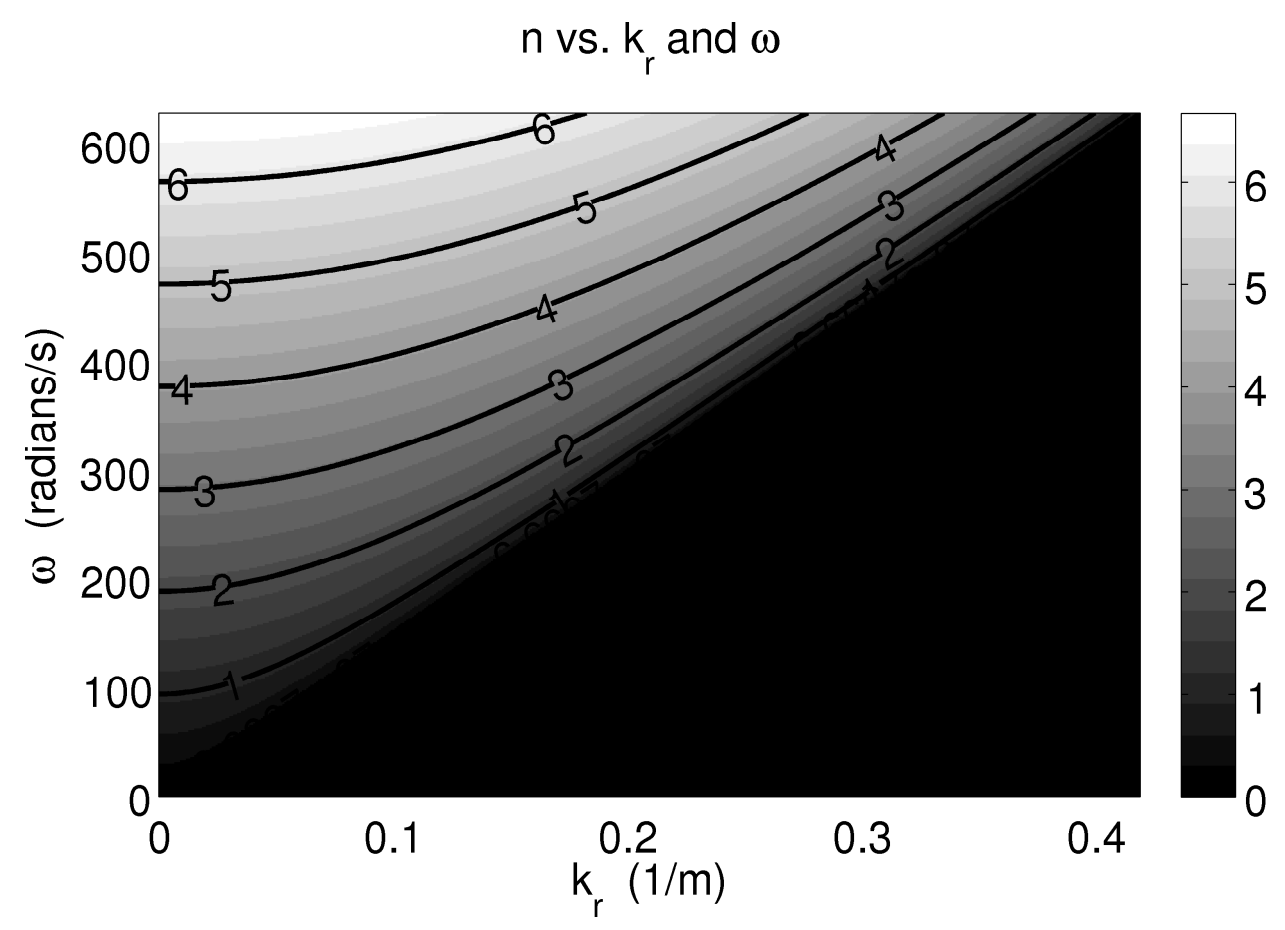

Figure 4.3: A contour plot of $n$ versus $k_{r}$ and $\omega$ for an ideal waveguide ( $d=50 \mathrm{~m}$, $c=1500 \mathrm{~m} / \mathrm{s}$ ). The solid black lines are contours at integer values of $n$, which in the WKB approximation correspond to the normal modes' horizontal wavenumbers.

$\beta(m, l, \omega)$ can then be interpreted as: -1 divided by the slope of the line connecting the points $\left(k_{r}, \omega, m\right)$ and $\left(k_{r}, \omega, l\right)$, divided by how the reciprocal of that slope changes with frequency. Or,

$$
-\frac{1 / \text { slope }}{\left(\frac{\partial(1 / \text { slope })}{\partial \omega}\right)_{m, l}}
$$

where the $m, l$ subscript indicates that the $m$ and $l$ values at which the points are located must stay constant as $\omega$ changes.

The discussion in the caption of Fig. 4.1 suggests that $\left(k_{r}(m, \omega)-k_{r}(l, \omega)\right)$ and its dependence on frequency does not strongly depend on the details $c(z)$ when 
$\left|k_{r}(m, \omega)-k_{r}(l, \omega)\right| \ll k_{\min }$ and $k_{r}(m, \omega)$ is not too close to $k_{\min }$, in some qualitative sense. So then one would expect that $\beta$ would also not depend on the details of the SSP under those circumstances in waveguides with a pressure-release bottoms. This is mentioned by Chuprov in [18, pp. 104].

\subsubsection{Approximating a finite difference with a continuous derivative}

The acoustic field in a waveguide is typically dominated by a few groups of modes with close mode numbers, for reasons discussed in Sec. 2.2.3. Because of that, $\beta$ is often defined only in terms of adjacent modes (see Sec. 2.3.3). Because the mode number $n$ is a monotonic function of the horizontal wavenumber $k_{r}$, a group of adjacent modes corresponds to a particular range of $k_{r}$ values. Here, we use that relation to approximate a finite difference in mode order with a continuous derivative in $n$.

If the curve $n\left(k_{r}, \omega\right)$ is approximately linear in $k_{r}$ between the points $\left(n, k_{r}(n, \omega)\right)$ and $\left(n+\Delta n, k_{r}(n+\Delta n, \omega)\right)$, then the finite difference between horizontal wavenumbers in Eq. (4.7) can be approximated by a continuous partial derivative:

$$
\lim _{\Delta n \rightarrow 0}\left(\frac{\left(k_{r}(n+\Delta n, \omega)-k_{r}(n, \omega)\right)}{\Delta n}\right)=\frac{\partial k_{r}(n, \omega)}{\partial n} .
$$

This is equivalent to the idea of expanding the group and phase slownesses around an average value for modes with adjacent $k_{r}$ values, as discussed in Sec. 2.3.3, [18], and [11, Sec. 6.7.2]. Most of the present analysis applies only to mode pairs with adjacent $k_{r}$ values, but Sec. 4.6 discusses how to calculate $\beta$ for mode pairs that have $k_{r}$ values that are far apart.

For adjacent modes, $\beta$ can then be written in terms of partial derivatives:

$$
\beta=-\frac{1}{\omega} \cdot \frac{\frac{\partial k_{r}(n, \omega)}{\partial n}}{\frac{\partial\left(\frac{\partial k_{r}(n, \omega)}{\partial n}\right)}{\partial \omega}}=-\frac{1}{\omega} \cdot \frac{\frac{\partial k_{r}(n, \omega)}{\partial n}}{\frac{\partial^{2} k_{r}(n, \omega)}{\partial n \partial \omega}} .
$$

\subsubsection{Implicit differentiation}

Calculating the partial derivatives in Eq. (4.10) is straightforward if one has an explicit solution for $k_{r}$ as a function of $n$ and $\omega$. But explicit solutions for $k_{r}(n, \omega)$ can 
only be obtained for a few cases such as modes in an ideal waveguide or surfacetrapped modes in an $n^{2}$-linear waveguide.

Therefore, the present analysis calculates the partial derivatives in Eq. (4.10) using implicit differentiation. Following the procedure for implicit differentiation in [31, Sec. 7.2], write Eq. (4.2) as

$$
n-\left(\phi+\Delta \phi_{\mathrm{dn}}+\Delta \phi_{\mathrm{up}}+1\right)=0 .
$$

Then define a function:

$$
f\left(k_{r}, n, \omega\right) \equiv n-\left(\phi+\Delta \phi_{\text {dn }}+\Delta \phi_{\text {up }}+1\right)=0
$$

which allows $k_{r}$ to be defined as an implicit function of $n$ and $\omega$ in regions where the derivative $\partial f / \partial k_{r}$ exists and is not equal to zero. The derivative $\partial f / \partial k_{r}$ exists and is not equal to zero except at a finite number of points, such as $k_{r}=k(0)$ and $k_{r}=k(d)$, as described in Appendix 4.A. So for our purposes $k_{r}$ may be considered an implicit function of $n$ and $\omega$.

The first step to calculate $\beta$ using implicit differentiation is to determine the first order partial derivatives of $k_{r}$ with respect to $n$ and with respect to $\omega$. These can be obtained using [31, Eq. 17].

$$
\begin{gathered}
\frac{\partial k_{r}}{\partial n}=-\frac{\partial f / \partial n}{\partial f / \partial k_{r}} \\
\frac{\partial k_{r}}{\partial \omega}=-\frac{\partial f / \partial \omega}{\partial f / \partial k_{r}}
\end{gathered}
$$

where $\partial f / \partial x$ indicates the result of differentiating $f$ with respect to the explicitly appearing variable $x$, holding all other explicitly appearing variables constant. More specifically, the partial derivatives with respect to $k_{r}$ on the right hand sides of Eqs. (4.13) and (4.14) should treat $k_{r}$ as if it were not a function of $n$ or $\omega$. See [31, Secs. 7.1-7.2] for details.

To obtain $\partial^{2} k_{r} /(\partial \omega \partial n)$ for the numerator of Eq. (4.10), the partial derivative of $\left(\frac{\partial k_{r}}{\partial n}\right)$ needs to be taken with respect to $\omega$. This can be done as follows:

1. Calculate $\frac{\partial k_{r}}{\partial n}$ using Eq. (4.13). 
2. Calculate $\frac{\partial}{\partial \omega}\left(\frac{\partial k_{r}}{\partial n}\right)$, where the partial derivative with respect to $\omega$ must treat $k_{r}$ as a function of $\omega$.

3. The result of step 2 will contain terms of the form $k_{r}^{\prime}(\omega)$. Those terms should be replaced with $\frac{\partial k_{r}}{\partial \omega}$ as calculated by Eq. (4.14).

The results can the be inserted into Eq. (4.10) obtain $\beta$.

As will be discussed in Sec.4.6, Eq. (4.14) can be used to determine the group speed of a mode at a particular $k_{r}$. However it cannot directly be used to obtain a dispersion curve for a particular mode, so the utility of such a calculation is not immediately apparent.

\section{Example}

As an example of how to use the method described in this section to calculate $\beta$, we will apply the method to an ideal waveguide with pressure-release boundaries (depth $d$, sound speed $c$ ):

$$
f\left(k_{r}, n, \omega\right)=n-\left(\frac{d}{\pi} \sqrt{\frac{\omega^{2}}{c^{2}}-k_{r}^{2}}\right) .
$$

The application of Eqs. (4.13) and (4.14) yield:

$$
\begin{gathered}
\frac{\partial k_{r}}{\partial n}=-\frac{\pi \sqrt{\frac{\omega^{2}}{c^{2}}-k_{r}^{2}}}{d \cdot k_{r}} \\
\frac{\partial k_{r}}{\partial \omega}=\frac{\omega}{c^{2} k_{r}} .
\end{gathered}
$$


Then we apply the steps to determine the mixed-partial derivative:

$$
\begin{aligned}
\frac{\partial}{\partial \omega}\left(\frac{\partial k_{r}}{\partial n}\right) & =\frac{\partial}{\partial \omega}\left(-\frac{\pi \sqrt{\frac{\omega^{2}}{c^{2}}-k_{r}(\omega)^{2}}}{d \cdot k_{r}(\omega)}\right) \\
& =\frac{\pi \omega\left(\omega k_{r}^{\prime}(\omega)-k_{r}(\omega)\right)}{d \cdot c^{2} k_{r}(\omega)^{2} \sqrt{\frac{\omega^{2}}{c^{2}}-k_{r}(\omega)^{2}}} \\
& =\frac{\pi \omega\left(\omega \frac{\omega}{c^{2} k_{r}}-k_{r}\right)}{d \cdot c^{2} k_{r}^{2} \sqrt{\frac{\omega^{2}}{c^{2}}-k_{r}^{2}}} .
\end{aligned}
$$

Inserting Eq. (4.16) and Eq. (4.18) into Eq. (4.10) yields:

$$
\beta=\frac{c^{2} k_{r}^{2}}{\omega^{2}}=\frac{k_{r}^{2}}{k^{2}}
$$

For modes far from cut-off $k_{r} \approx k$ leading to $\beta \approx 1$, which matches the result in Sec. 2.3.2. Note that the estimate of $\beta$ is a function of $k_{r}$ instead of begin a function of $n$ because the differentiation was performed implicitly.

\subsection{Exploiting dependence on $\omega$}

In the previous section it was shown that a mixed second-order partial derivative of $k_{r}$ with respect to $n$ and $\omega$ was required to calculate $\beta$ [Eq. (4.10)], implying that $\beta$ depended on two independent variables. In this section we show that under certain circumstances, $\phi, \Delta \phi_{\mathrm{dn}}$ and $\Delta \phi_{\text {up }}$ depend on $k_{r}$ and $\omega$ in such a way that the mixed second-order partial derivative can be expressed as a non-mixed (regular) second-order derivative, meaning that $\beta$ depends only on one independent variable. It should be noted that this result is not entirely new: In [18] Chuprov mentions that under the WKB approximation, the functional dependence of the group speed on the phase speed - a quantity that can be used to define $\beta$ - does not depend on the frequency under certain circumstances.

We begin by noting that a plot of $\phi\left(k_{r}, \omega\right)$ will look like a scaled down version of a plot of $\phi\left(k_{r}, \omega+\Delta \omega\right)$; both axes shrink uniformly. Furthermore, the shape of $\Delta \phi_{\mathrm{dn}}=\phi_{b}$ also scales with frequency, but the scaling only occurs on the $\omega$ axis. 
This scaling suggests that the shape of $\phi\left(k_{r}, \omega\right)$ and $\phi_{b}\left(k_{r}, \omega\right)$ at one frequency as a function of $k_{r}$ provides sufficient information to take partial derivatives with respect to either $k_{r}$ or $\omega$ at any frequency. We now show that $\phi\left(k_{r}, \omega\right)$ can be written as $\omega \cdot \phi\left(1, k_{r} / \omega\right)$ and $\Delta \phi_{\mathrm{dn}}\left(k_{r}, \omega\right)$ can be written as $\Delta \phi_{\mathrm{dn}}\left(k_{r} / \omega\right)$.

First, factor an $\omega$ out of $k_{z}(z)$ :

$$
\begin{aligned}
\phi\left(k_{r}, \omega\right) & =\frac{1}{\pi} \int_{z_{1}}^{z_{2}}\left(\sqrt{\left(\frac{\omega}{c(z)}\right)^{2}-k_{r}^{2}}\right) d z \\
& =\frac{\omega}{\pi} \int_{z_{1}}^{z_{2}}\left(\sqrt{\frac{1}{c(z)^{2}}-\left(\frac{k_{r}}{\omega}\right)^{2}}\right) d z \\
& =\omega \cdot \phi\left(\frac{k_{r}}{\omega}, 1\right) \\
& =\omega \cdot \phi_{1}\left(\frac{k_{r}}{\omega}\right)
\end{aligned}
$$

where

$$
\phi_{1}\left(\frac{k_{r}}{\omega}\right) \equiv \frac{1}{\pi} \int_{z_{1}}^{z_{2}}\left(\sqrt{\frac{1}{c(z)^{2}}-\left(\frac{k_{r}}{\omega}\right)^{2}}\right) d z
$$

Note that $\left(\frac{k_{r}}{\omega}\right)$ is the inverse of the phase speed, which is known as the phase slowness $S_{p}$.

$$
S_{p} \equiv \frac{k_{r}}{\omega}
$$

The remainder of this chapter will denote $\left(\frac{k_{r}}{\omega}\right)$ by $S_{p}$ to simplify notation, but it is important to keep in mind the $S_{p}$ is a function of $\omega$ when taking derivatives with respect to $\omega$.

Next we show that $\phi_{b}$, the phase of the reflection coefficient between twohomogeneous half-spaces, and thus $\phi_{\mathrm{dn}}$ can be written as a function of $S_{p}$. Following [33, Eq. 1.45 and Eq. 2.125],

$$
\text { Reflection coefficient }=R=\frac{Z_{2}-Z_{1}}{Z_{1}+Z_{2}}
$$


where

$$
\begin{aligned}
Z_{i} & =\frac{\rho_{i} c_{i}}{\sin \theta_{i}} \\
& =\frac{k \rho_{i} c_{i}}{k_{z}} \\
& =\frac{\left(\omega / c_{i}\right) \rho_{i} c_{i}}{\sqrt{\left(\omega / c_{i}\right)^{2}-k_{r}^{2}}} \\
& =\frac{\rho_{i}}{\sqrt{\left(1 / c_{i}\right)^{2}-\left(k_{r} / \omega\right)^{2}}} \\
& =\frac{\rho_{i}}{\sqrt{\left(1 / c_{i}\right)^{2}-S_{p}^{2}}} .
\end{aligned}
$$

Then $R$ can be written as a function of $S_{p}$, and so can $\Delta \phi_{\mathrm{dn}}$.

To see that $\Delta \phi_{\text {up }}$ (and $\Delta \phi_{\text {dn }}$ in the case of a vacuum bottom) can be written as function of $S_{p}$, note that the value of $\Delta \phi_{\text {up }}\left(k_{r}, \omega\right)$ as defined in Sec. 4.2 depends only on the point at which $k_{r}=\frac{\omega}{c(0)}$, or equivalently at $S_{p}=\frac{1}{c(0)}$.

Having written $\phi, \Delta \phi_{\text {up }}$, and $\Delta \phi_{\mathrm{dn}}$ as function of $\left(k_{r} / \omega\right)$, the waveguide invariant $\beta$ can be calculated by writing Eq. (4.12) as

$$
\begin{aligned}
0 & =f\left(k_{r}, n, \omega\right) \\
& =n-\left(\omega \cdot \phi_{1}\left(\frac{k_{r}}{\omega}\right)+\Delta \phi_{\mathrm{dn}}\left(\frac{k_{r}}{\omega}\right)+\Delta \phi_{\mathrm{up}}\left(\frac{k_{r}}{\omega}\right)+1\right)
\end{aligned}
$$

and then following the steps in Sec. 4.2.2 to calculate $\beta$ from $f\left(k_{r}, n, \omega\right)$. The resulting expressions are lengthy and difficult to manipulate by hand, so the author used the computer algebra system Mathematica. See Appendix 4.B for details. The final result can be simplified down to:

$$
\beta\left(S_{p}, \omega\right)=-\frac{\left(\omega \phi_{1}^{\prime}\left(S_{p}\right)+\Delta \phi_{\mathrm{dn}}^{\prime}\left(S_{p}\right)\right)^{2}}{\Delta \phi_{\mathrm{dn}}^{\prime}\left(S_{p}\right)^{2}+\omega \Delta \phi_{\mathrm{dn}}^{\prime}\left(S_{p}\right) \phi_{1}^{\prime}\left(S_{p}\right)+\omega \phi_{1}\left(S_{p}\right)\left(\Delta \phi_{\mathrm{dn}}^{\prime \prime}\left(S_{p}\right)+\omega \phi_{1}^{\prime \prime}\left(S_{p}\right)\right)}
$$

where the ' denotes a derivative with respect to the argument $S_{p}=\frac{k_{r}}{\omega}$. Note that $\Delta \phi_{\mathrm{dn}}^{\prime}$ is related to the "ray displacement" discussed in [11, Sec. 4.4].

When $S_{p}>1 / c(d)$ (i.e, when the mode has a lower turning point) or if $\Delta \phi_{\mathrm{dn}}$ represents a vacuum bottom, then the derivative of $\Delta \phi_{\mathrm{dn}}$ with respect to $S_{p}$ is zero 
everywhere except possibly at one point, and Eq. (4.26) can be reduced to

$$
\beta\left(S_{p}\right)=-\frac{\phi_{1}^{\prime}\left(S_{p}\right)^{2}}{\phi_{1}\left(S_{p}\right) \phi_{1}^{\prime \prime}\left(S_{p}\right)} .
$$

This expression is remarkably simple given the complexity of its derivation, which is shown in Appendix 4.B. When it's valid, Eq. (4.27) provides an direct relationship between the SSP and the value of $\beta$ at all horizontal wavenumbers and all frequencies though a single function of a single variable: $\phi_{1}\left(S_{p}\right)$. It should be noted that Eq. 4.27 is equivalent to [14, Eq. 24].

\subsection{Interpretation and implementation}

Eq. (4.26) reveals that the shape of $\phi_{1}\left(S_{p}\right)$ and $\phi_{\mathrm{dn}}\left(S_{p}\right)$ provides sufficient information to calculate the value of $\beta$ for any $k_{r}$ and $\omega$. Eq. (4.27) reveals that when $S_{p}>1 / c(d)$ or when the bottom is approximated as being a vacuum, $\beta$ depends only the ratio of $k_{r}$ to $\omega$, as opposed to depending on each variable independently.

To relate the value of $\beta$ to the SSP $c(z)$ and $S_{p}$, some properties of $\phi_{1}\left(S_{p}\right)$ will now be discussed. Let $c_{\min }$ and $c_{\max }$ be the minimum and maximum sound speed in the water column, not including the seafloor. $\phi_{1}\left(S_{p}\right)$ can be broken up into two regions, the first being $\left(0<S_{p}<1 / c_{\max }\right)$ which corresponds to SRBR modes (see Sec. 2.2.2). And the second being $\left(1 / c_{\max }<S_{p}<1 / c_{\min }\right)$ which corresponds to non-SRBR modes. In both regions $\phi_{1}\left(S_{p}\right)$ is a positive and monotonically decreasing function.

Modes with horizontal wavenumbers in the first region do not have any turning points and "feel" the top and bottom boundary of the waveguide. In this region $\phi_{1}\left(S_{p}\right)$ and its derivatives can be approximated to arbitrary accuracy by breaking the water-column up into several small iso-velocity segments, or equivalently, ap- 
proximating Eq. (4.21) with Riemann Sum:

$$
\begin{aligned}
& \phi_{1}\left(S_{p}\right)=\sum_{i=1}^{N} \phi_{1, i}\left(S_{p}\right)=\frac{1}{\pi} \sum_{i=1}^{N} d_{i} \sqrt{\frac{1}{c_{i}^{2}}-S_{p}^{2}} \\
& \phi_{1}^{\prime}\left(S_{p}\right)=\frac{1}{\pi} \sum_{i=1}^{N} \frac{-S_{p} d_{i}}{\sqrt{\frac{1}{c_{i}^{2}}-S_{p}^{2}}} \\
& \phi_{1}^{\prime \prime}\left(S_{p}\right)=\frac{1}{\pi} \sum_{i=1}^{N}-\left(\frac{S_{p}^{2} d_{i}}{\left(\frac{1}{c_{i}^{2}}-S_{p}^{2}\right)^{3 / 2}}+\frac{d_{i}}{\sqrt{\frac{1}{c_{i}^{2}}-S_{p}^{2}}}\right)
\end{aligned}
$$

where $\phi_{1, i}\left(S_{p}\right)$ represents the contribution from the $i$-th segment, and $d_{i}$ is the depth of the $i$-th segment. For $\left(S_{p}<1 / c_{\max }\right)$, every term in Eq. (4.28a) is a positive but monotonically decreasing function with negative concavity, so $\phi_{1}^{\prime \prime}\left(S_{p}\right)<0$, as can be verified by inspection of Eq. (4.28c). Inserting the sign of $\phi_{1}\left(S_{p}\right)$ and $\phi_{1}^{\prime \prime}\left(S_{p}\right)$ into Eq. (4.27) reveals that $\beta>0$ for all SRBR modes in a waveguide with a pressure-release boundaries, regardless of the SSP.

For non-SRBR modes $\left(1 / c_{\max }<S_{p}<1 / c_{\min }\right)$, Eqs. (4.28b) and (4.28c) are incorrect because they do not take into account that the limits of the definite integral of $k_{z}(z)$ in Eq. (4.21) - the turning points - are themselves functions of $S_{p}{ }^{3}$ Therefore in SSP segments where a turning point exists, $c(z)$ must be represented with a segment that is not iso-velocity so that the derivative with respect to $S_{p}$ can take into account that the limits of the integral in Eq. (4.21) are functions of $S_{p}$. For this we use $n^{2}$-linear segments ([33, Sec. 2.5.1] and [5]), which can be analytically integrated and are very close to linear for the SSPs typically encountered in ocean acoustics. A segment with an $n^{2}$-linear SSP is one of the form

$$
c_{i}(z)=\frac{1}{\sqrt{a_{i} z+b_{i}}}
$$

where $a_{i}$ and $b_{i}$ are chosen to interpolate the SSP. Each segment can have its own coordinate system, so without loss of generality let $a_{i}<0$.

\footnotetext{
${ }^{3}$ See "Leibniz Integral Rule" in any introductory Calculus textbook. Note that although the value of $\phi_{1}^{\prime}\left(S_{p}\right)$ is not affected by the fact that the integration limits are functions of $S_{p}$ because $k_{z}\left(z, S_{p}\right)=0$ when $\left(z, S_{p}\right)$ corresponds to a turning point, the value of $\phi_{1}^{\prime \prime}\left(S_{p}\right)$ is affected.
} 
Inserting Eq. (4.29) into Eq. (4.21) yields

$$
\begin{aligned}
\phi_{1, i}\left(S_{p}\right) & =\frac{1}{\pi} \int_{0}^{d_{i}}\left(\sqrt{\frac{1}{c(z)^{2}}-S_{p}^{2}}\right) d z \\
& =\frac{2}{3 \pi a_{i}}\left(\left(b_{i}+a_{i} d_{i}-S_{p}^{2}\right)^{3 / 2}-\left(b_{i}-S_{p}^{2}\right)^{3 / 2}\right) .
\end{aligned}
$$

For a segment that contains a turning point, $\sqrt{b_{i}+a_{i} d_{i}-S_{p}^{2}}=0$ at that turning point and thus

$$
\phi_{1, i}\left(S_{p}\right)=\frac{-2}{3 \pi a_{i}}\left(b_{i}-S_{p}^{2}\right)^{3 / 2} .
$$

To represent an arbitrary SSP, one can use any combination of segments that are iso-velocity or $n^{2}$-linear (or any $c(z)$ for which there is a closed form expression of Eq. (4.21)), as long as the segments that contain turning points are not iso-velocity.

$\phi_{1}\left(S_{p}\right), \phi_{1}^{\prime}\left(S_{p}\right), \phi_{1}^{\prime \prime}\left(S_{p}\right)$, which are needed to calculate $\beta$, can then be calculated quasi-analytically in the sense that the derivatives are taken analytically for each term of the summation that represents $c(z)$, but the summations themselves may be computed numerically if there are too many terms to handle analytically. In the case of a waveguide with a bottom half-space, the function $\phi_{b}\left(S_{p}\right)$ is also required to calculate $\beta$ and its derivatives can calculated analytically from Eq. (4.23).

Many waveguides can be sufficiently well represented with just a few segments and a bottom half-space, and thus $\beta$ can be computed purely analytically in those cases. In all waveguides, regardless of how complicated the SSP is, $\phi_{1}$ has a straightforward meaning in the sense that it is easy to qualitatively understand how the details of the SSP affect $\phi_{1}$.

\subsection{Examples}

This section applies the methods described in Secs. $4.2-4.4$ to calculating $\beta$ in waveguides with several commonly analyzed SSPs: iso-velocity, a single $n^{2}$-linear segment, and a deep-water Munk SSP. For each SSP, $\beta$ is plotted for a vacuum bottom and for a fluid halfspace bottom. In addition $k(z), \phi_{1}$ and $\phi_{\mathrm{dn}}$ are plotted on an $\mathrm{x}$-axis common with the plots of $\beta$ so that their relationship to $\beta$ can be 
easily seen. In all cases that have a fluid bottom halfspace, $c_{\text {bottom }}=1650 \mathrm{~m} / \mathrm{s}$, $\rho_{\text {bottom }}=1.5 \rho_{\text {water }}$.

For comparison, the normal mode program Kraken [48] was also used to calculate the value of $\beta$ using the same environmental parameters. $\beta$ was calculated from Kraken using Eq. 2.27, using only adjacent mode numbers.

All calculations in Kraken used an acoustic source frequency of $250 \mathrm{~Hz}$ except for the deep-water Munk case which used a frequency of $75 \mathrm{~Hz}$. As the frequency increases, the agreement between Kraken and the methods described in this chapter tends to improve, as would be expected because the accuracy of the WKB approximation increases with frequency as does the accuracy of approximating the finite difference in mode order with a continuous derivative. This agreement provides a sanity check on the validity of the present analysis.

\subsubsection{Iso-velocity SSP: ideal and Pekeris waveguides}

An iso-velocity SSP with a vacuum bottom is known as an ideal waveguide. Although $\beta$ for an ideal waveguide can be calculated analytically using Eq. (2.15) because explicit expressions for $k_{r}(m, \omega)$ can be obtained (See Sec. 2.3.2), the result is reproduced here for illustrative purposes. For an ideal waveguide

$$
\phi_{1}\left(S_{p}\right)=\frac{d}{\pi} \sqrt{\frac{1}{c^{2}}-S_{p}^{2}}
$$

Calculating $\phi_{1}^{\prime}\left(S_{p}\right)$ and $\phi_{1}^{\prime \prime}\left(S_{p}\right)$ and inserting into Eq. (4.27) yields:

$$
\beta=c^{2} S_{p}^{2}
$$

As $S_{p} \rightarrow 1 / c$, which happens as modes move away from being cut off, $\beta \rightarrow 1$, as shown in Fig. 4.4(c).

An iso-velocity SSP with a bottom fluid halfspace is known as a Pekeris waveguide. $\phi_{1}\left(S_{p}\right)$ is the same as that for an ideal waveguide but the derivatives of $\phi_{\mathrm{dn}}\left(S_{p}\right)$ are non-zero, so Eq. (4.26) must be used instead of Eq. (4.27). $\phi_{\mathrm{dn}}\left(S_{p}\right)$ for a bottom that is denser and has a faster $c$ than the water just above it always has the same qualitative shape, shown in Fig. 4.4(b). $\phi_{\mathrm{dn}}^{\prime}\left(S_{p}\right)$ and $\phi_{\mathrm{dn}}^{\prime \prime}\left(S_{p}\right)$ can be calculated analytically in a straightforward manner, although the result is a lengthy 
expression. Eq. (4.26) can then be used to obtain an analytic estimate of $\beta$ as a function of $S_{p}$. In the case of a Pekeris waveguide, the WKB approximation is the exact solution, so the only approximation made in calculating $\beta$ is approximating the finite difference in mode number with a continuous derivative. The reason one can obtain an analytic estimate of $\beta$ in a Pekeris waveguide despite not being able to analytically solve for $k_{r}(m, \omega)$ is that the resulting $\beta$ is a continuous function of $S_{p}$ instead of being an explicit function of mode number. Figure 4.4(c) shows a plot of $\beta$ for a Pekeris waveguide. 


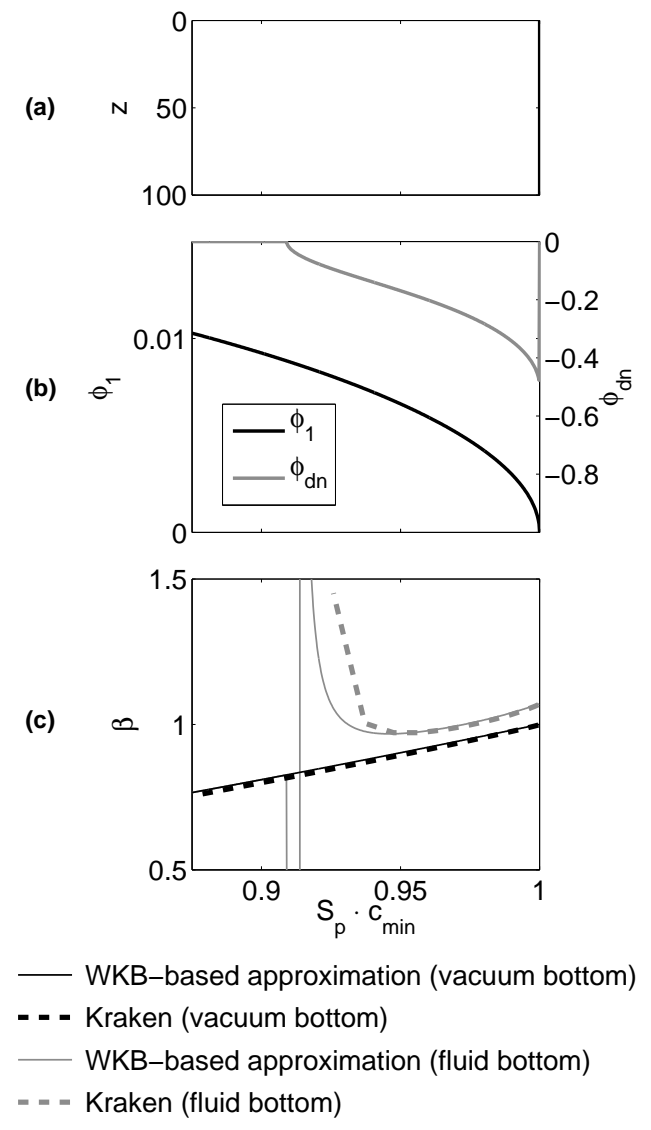

Figure 4.4: The waveguide invariant $\beta$ for an ideal and a Pekeris waveguide, and the functions used to calculate $\beta$, all plotted on a common $\mathrm{x}$-axis scale (the phase slowness $S_{p}$ normalized by the minimum sound speed in the water column). (a) $k(z)=\frac{\omega}{c(z)}$, which in this case is a constant and equal to 1 because of the normalized scale. (b) The $\phi_{1}\left(S_{p}\right)$ associated with $k(z)$ (black), and $\phi_{\mathrm{dn}}\left(S_{p}\right)$ for the bottom fluid halfspace (gray). (c) $\beta$ versus phase slowness. For the ideal waveguide (vacuum bottom) the WKB-based approximation of $\beta$ closely matches the value calculated from Kraken. For the Pekeris waveguide (fluid bottom) there is also good agreement between Kraken and the WKB-based approximation. For the fluid bottom, the WKB-based approximation of $\beta$ is unbounded at the value of $S_{p}$ corresponding to the critical angle because the derivative of $\phi_{\mathrm{dn}}\left(S_{p}\right)$ is infinite. 


\subsection{2 $n^{2}$-linear waveguide}

The $n^{2}$-linear waveguide used in this section has $c(0)=1500 \mathrm{~m} / \mathrm{s}$ and $c(d)=1550$ $\mathrm{m} / \mathrm{s}$, and $d=100 \mathrm{~m}$. The $k(z)$ associated with $c(z)$ is shown in Fig. 4.5(a).

\section{Surface-trapped modes}

Surface-trapped modes are modes that have $S_{p}>1 / c(d)$ in an upward refracting SSP. Using Eqs. (4.31) and (4.25), one can obtain an explicit solution for $k_{r}(m, \omega)$, use a Taylor series expansion similar to that in [11, Sec. 6.7.2] and then use Eq. (2.15) to see that $\beta \approx-3$ for modes far from cut-off. Alternatively one can use the method in Sec. 4.3. Using Eq. (4.31) to represent $\phi_{1}$ and inserting $\phi_{1}$ into Eq. (4.27) yields:

$$
\beta=\frac{3 S_{p}^{2}}{b-2 S_{p}^{2}}
$$

Surface-trapped modes far from cutoff have a phase slowness of $S_{p} \approx 1 / c(0)=$ $\sqrt{b}$. Inserting that into Eq. (4.34) results in $\beta=-3$.

For $S_{p}>1 / c(d)$ the concavity of $\phi_{1}$ is positive, as shown in Fig. 4.5(b). This is because as $S_{p}$ decreases, the integrand increases in value and the limits of the integral move further apart. The result is that $\beta$ is a negative number for $S_{p}>$ $1 / c(d)$ as can be seen in Fig. 4.5(c).

This analysis for surface-trapped modes also applies to waterborne modes in a two-segment ducted waveguide where each side of the duct has an $n^{2}$-linear SSP, because such modes have a $\phi_{1}$ equivalent to that of the surface-trapped modes discussed above, and so those modes will also have $\beta \approx-3$ [26].

\section{SRBR modes}

SRBR modes in this upward refracting environment have $S_{p}>1 / c(d)$. Based on the qualitative argument in Fig. 4.1, one would expect that $\beta$ for the SRBR modes should be similar to $\beta$ in an iso-velocity waveguide when $(k(0)-k(d)) /(k(0)-$ $\left.k_{r}\right) \ll 1$. An explicit analytic solution for $k_{r}(m, \omega)$ of these modes is either intractable or does not exist. However $\beta$ can be calculated with fairly simple expressions using Eqs. (4.30) and (4.27). $\beta$ for a vacuum and a fluid halfspace bottom are shown in Fig. 4.5(c). 

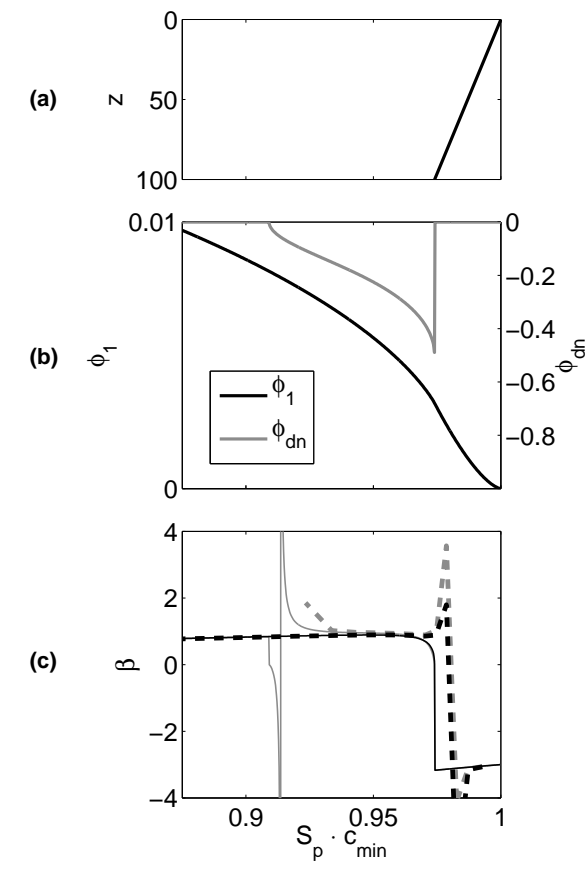

\footnotetext{
- WKB-based approximation (vacuum bottom)

- - - Kraken (vacuum bottom)

- WKB-based approximation (fluid bottom)

- - - Kraken (fluid bottom)
}

Figure 4.5: The waveguide invariant $\beta$ for an $n^{2}$-linear waveguide, and the functions used to calculate $\beta$ plotted on a common x-axis (the phase slowness $S_{p}$ normalized by the minimum sound speed in the water column). (a) Normalized $k(z)$. (b) The $\phi_{1}\left(S_{p}\right)$ associated with $k(z)$ (black), and $\phi_{\mathrm{dn}}\left(S_{p}\right)$ for the bottom fluid halfspace (gray). Note that the concavity of $\phi_{1}\left(S_{p}\right)$ changes when $S_{p}=1 / c(d)$, which results in a sign change of $\beta$ at that point. (c) $\beta$ for the vacuum and fluid bottom $n^{2}$-linear waveguides. The WKB-based approximation is in good agreement with Kraken everywhere except in the region near $S_{p}=1 / c(d)=0.974 \cdot c_{\min }$ which is where the mode's turning point is close to the bottom and the WKB approximation is inaccurate. For $S_{p}>1 / c(d)$ the modes are surface-trapped, $\phi_{1}$ has positive concavity, and $\beta \approx-3$. For $S_{p}<1 / c(d)$, which corresponds to bottom-interacting modes, $\beta \approx 1$. The effect of including a bottom fluid halfspace instead of a vacuum is small, except for modes close to cutoff (modes with $k_{r}$ values near the critical angle $k_{r}$ value) because $\phi_{\mathrm{dn}}^{\prime}\left(S_{p}\right)$ becomes unbounded at that point. 


\subsubsection{Quasi-analytic results for a deep-water Munk profile}

A deep-water waveguide with a Munk SSP [33, Sec. 5.6] can be approximated with $n^{2}$-linear segments. Fig. 4.6(c) shows $\beta$ for $5000 \mathrm{~m}$ deep waveguide with a Munk SSP, for both a vacuum and a fluid halfspace bottom. The $\phi_{1}$ used for calculating $\beta$ was computed with Eq. (4.30) using 1000 segments to represent $c(z)$. 


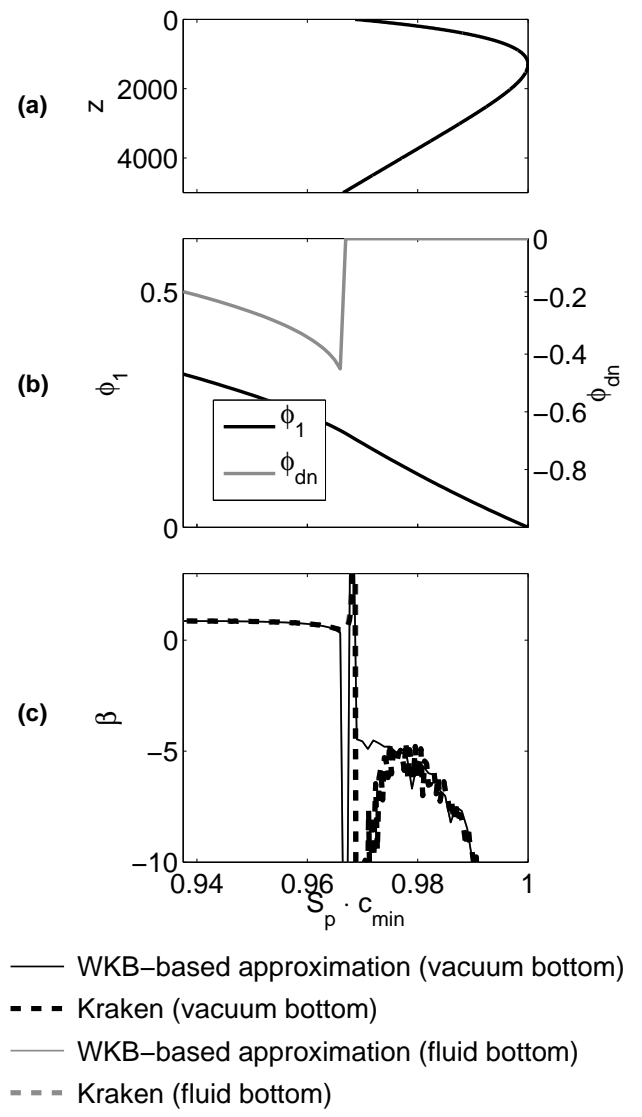

Figure 4.6: The waveguide invariant $\beta$ for a deep-water Munk SSP, and the functions used to calculate $\beta$ plotted on a common x-axis (the phase slowness $S_{p}$ normalized by the minimum sound speed in the water column). (a) Normalized $k(z)$. (b) The $\phi_{1}\left(S_{p}\right)$ associated with $k(z)$ (black), and $\phi_{\mathrm{dn}}\left(S_{p}\right)$ for the bottom fluid halfspace (gray). (c) $\beta$ for the Munk SSP with vacuum and fluid bottoms. In general there is good agreement between the WKB-based approximation and Kraken except at $S_{p}$ values where the mode's turning point is near a boundary and the WKB approximation is inaccurate. In regions where modes are non-SRBR $\left(S_{p} \cdot c_{\min }>0.968\right)$, the concavity of $\phi_{1}\left(S_{p}\right)$ is negative and so $\beta$ is positive. For $S_{p}$ values corresponding to top- and bottom-interacting modes, $\beta$ is between about 0.5 and 1 . The effect of including bottom fluid halfspace instead of a vacuum is negligible, which is why no gray lines can be seen. 


\subsubsection{Realistic shallow water waveguide}

Section 3.4.2 presented some experimentally measured waveguide invariant striations from a shallow water waveguide (see that section for details). In this subsection, we calculate the value of $\beta$ for that environment (i.e. for the SSP in Fig. 3.5, assuming $c_{\text {bottom }}=1500 \mathrm{~m} / \mathrm{s}$ ). It can be seen that $\beta$ has a value slightly less than one for the SRBR modes, and that $\beta$ varies greatly for non-SRBR modes. 
(a)

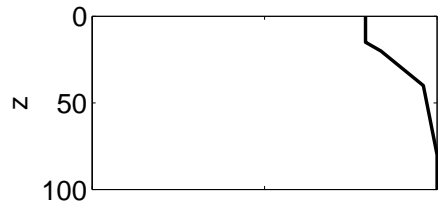

(b) $e^{-}$

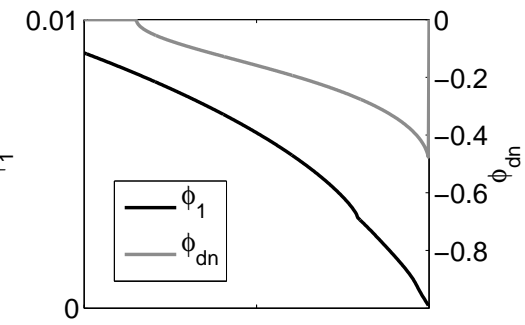

(c)

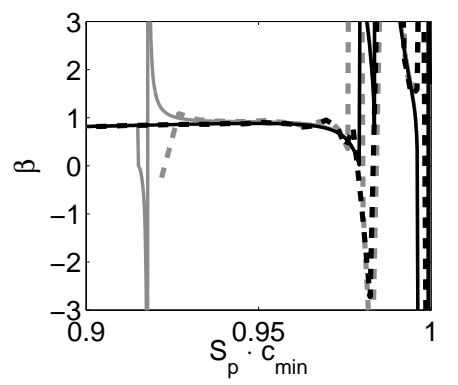

\footnotetext{
WKB-based approximation (vacuum bottom)

- - - Kraken (vacuum bottom)

WKB-based approximation (fluid bottom)

$=-=$ Kraken (fluid bottom)
}

Figure 4.7: The waveguide invariant $\beta$ for a realistic shallow water waveguide, and the functions used to calculate $\beta$ plotted on a common $\mathrm{x}$-axis (the phase slowness $S_{p}$ normalized by the minimum sound speed in the water column). The SSP for this figure comes from Fig. 3.5 (a) Normalized $k(z)$. (b) The $\phi_{1}\left(S_{p}\right)$ associated with $k(z)$ (black), and $\phi_{\mathrm{dn}}\left(S_{p}\right)$ for the bottom fluid halfspace (gray). (c) $\beta$ for the realistic shallow water SSP with vacuum and fluid bottoms. In regions where modes are $\operatorname{SRBR}\left(S_{p} \cdot c_{\min }<0.98\right), \beta$ is slightly less than 1 . For $S_{p}$ values corresponding SRBR modes $\left(S_{p} \cdot c_{\min }>0.98\right)$, the value of $\beta$ varies greatly. 


\subsection{Calculating $\beta$ for non-adjacent modes}

The definition of $\beta$ in its most general form, Eq. (2.15), is for a pair of modes whose $k_{r}$ values are not necessarily adjacent to one another. The methods for calculating $\beta$ in Secs. 4.2 - 4.5 are valid only for mode pairs that have $k_{r}$ values that are close enough together so that the curve $\phi_{1}\left(S_{p}=k_{r} / \omega\right)$ is approximately linear between the two modes' $S_{p}$ values. This restriction on the validity is because the finite difference of the modes' $k_{r}$ values was approximated to be infinitesimally small (i.e., a derivative). Because of this, $\beta$ depended only on a single $k_{r}$ value. The justification for such an approach was discussed in the beginning of Sec. 4.2.1.

However, some components of the acoustic intensity field $I(r, \omega)$ will be the result of mode pairs that have $S_{p}$ values that are far apart (non-adjacent). When calculating $\beta$ for such mode pairs, the finite difference between the modes' $k_{r}$ values cannot be approximated by a derivative as was done in Secs. 4.2 - 4.5. However some of the analysis from Secs. 4.2 and 4.3 is still valid for a pair of non-adjacent modes, and can be applied to calculate $\beta$ for non-adjacent modes. The resulting $\beta$ will depend explicitly on the two modes' $S_{p}$ values, and implicitly on the two modes' modal numbers.

Using Eq. (2.15) as a definition for $\beta$, Eq. (4.25) can be inserted into Eq. (4.14) to implicitly the calculate derivatives with respect to $\omega$ in the denominator Eq. (2.15).

$$
\frac{\partial k_{r}}{\partial \omega}=\frac{\phi_{1}\left(\frac{k_{r}}{\omega}\right)-\frac{k_{r}}{\omega} \phi_{1}^{\prime}\left(\frac{k_{r}}{\omega}\right)-\frac{k_{r}}{\omega^{2}} \phi_{\mathrm{dn}}^{\prime}\left(\frac{k_{r}}{\omega}\right)}{-\phi_{1}^{\prime}\left(\frac{k_{r}}{\omega}\right)-\frac{1}{\omega} \phi_{\mathrm{dn}}^{\prime}\left(\frac{k_{r}}{\omega}\right)}
$$

If the bottom fluid halfspace is approximated as being a vacuum, then $\phi_{\mathrm{dn}}^{\prime}=0$ and

$$
\begin{aligned}
\frac{\partial k_{r}}{\partial \omega} & =-\frac{\phi_{1}\left(S_{p}\right)+S_{p} \phi_{1}^{\prime}\left(S_{p}\right)}{\phi_{1}^{\prime}\left(S_{p}\right)} \\
& =\left(S_{p}-\frac{\phi_{1}\left(S_{p}\right)}{\phi_{1}^{\prime}\left(S_{p}\right)}\right) .
\end{aligned}
$$

Note that this is the group slowness. It is worth mentioning that Eq. (4.36) is identical to [45, Eq. 2.14.12], where it appears in the context of ray theory and $S_{p}$ was defined as the slowness of a ray at its turning point, as opposed to the definition used here which is in terms of the modal phase slowness and thus explicitly takes 
into account frequency dependence.

Eq. (4.35) or (4.36) can then be inserted into Eq. (2.15) to calculate $\beta$ for a pair of modes with $S_{p}$ values that are not adjacent. When the bottom is a vacuum:

$$
\begin{aligned}
\beta\left(k_{r, m}, k_{r, l}, \omega\right) & =-\frac{1}{\omega} \cdot \frac{\left(k_{r, m}-k_{r, l}\right)}{\frac{\partial\left(k_{r, m}-k_{r, l}\right)}{\partial \omega}} \\
& =-\frac{\frac{1}{\omega}\left(k_{r, m}-k_{r, l}\right)}{\left(S_{p, m}-S_{p, l}\right)+\left(\frac{\phi_{1}\left(S_{p, l}\right)}{\phi_{1}^{\prime}\left(S_{p, l}\right)}-\frac{\phi_{1}\left(S_{p, m}\right)}{\phi_{1}^{\prime}\left(\frac{k_{r, m}}{\omega}\right)}\right)} \\
& =-\frac{\left(S_{p, m}-S_{p, l}\right)}{\left(S_{p, m}-S_{p, l}\right)+\left(\frac{\phi_{1}\left(S_{p, l}\right)}{\phi_{1}^{\prime}\left(S_{p, l}\right)}-\frac{\phi_{1}\left(S_{p, m}\right)}{\phi_{1}^{\prime}\left(S_{p, m}\right)}\right)} .
\end{aligned}
$$

$\beta\left(S_{p, m}, S_{p, l}\right)$ can then be plotted as a function of the two modes' phase slownesses. Although the specific $S_{p, m}$ and $S_{p, l}$ values that correspond to propagating modes are unknown unless one explicitly solves for them, one can determine the regions of $\beta\left(S_{p, m}, S_{p, l}\right)$ that correspond to SRBR modes and non-SRBR modes.

Fig. 4.8 shows $\beta\left(S_{p, m}, S_{p, l}\right)$ for the same $n^{2}$-linear waveguide with a vacuum bottom used in the previous section, calculated using Eq. (4.37) and using Kraken for comparison. A fluid bottom could be taken into account inserting Eq. (4.35) into Eq. (2.15).

\subsection{Chapter summary}

Knowing the value of $\beta$ is critical for many applications of the waveguide invariant, such as the passive range estimation presented in Ch. 3. Under the WKB approximation, $\beta$ can be determined solely from the phase of the bottom Rayleigh reflection coefficient as a function of the phase slowness and from the integrated phase of $k_{z}$ in the water column as a function of the phase slowness, $\phi_{1}\left(S_{p}\right)$. If the waveguide has a bottom fluid halfspace, $\beta$ depends on $k_{r}$ and $\omega$ independently and can be calculated using Eq. (4.26). The use of implicit differentiation allows for analytic estimates of $\beta$ in waveguides whose horizontal wavenumbers cannot be explicitly solved for analytically. When the bottom is approximated as being a vacuum, $\beta$ depends only on the ratio of $k_{r}$ to $\omega$ (phase slowness), has a simple 
(a)

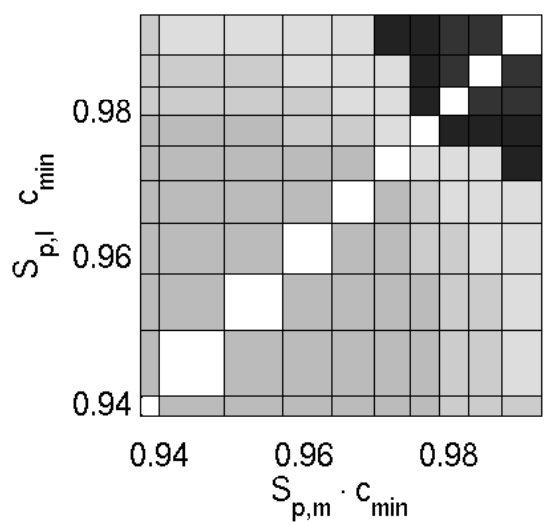

(b)

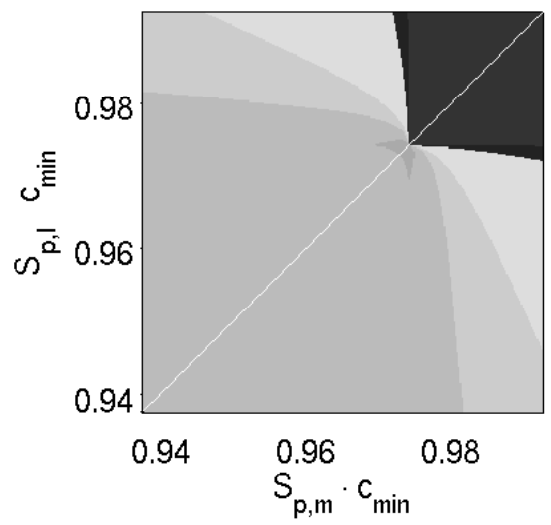

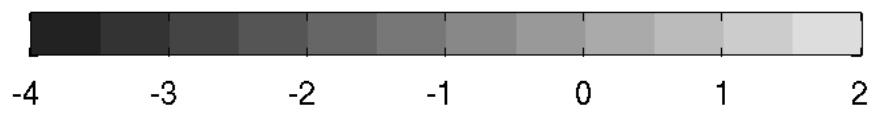

Figure 4.8: Plots of the waveguide invariant $\beta$ for non-adjacent modes versus normalized phase slowness $\left(S_{p} \cdot c_{\min }\right)$ for each mode $\left(S_{p . m}\right.$ and $\left.S_{p . l}\right)$. These plots are from the same $n^{2}$-linear SSP used for Fig. 4.5, with a vacuum bottom and were calculated from (a) Kraken and (b) Eq. (4.37). The white areas along the diagonal correspond to $m=l$ in Eq. (2.15) and are thus undefined. A diagonal "slice" just off the line $S_{p, m}=S_{p, l}$ corresponds to adjacent modes, and is the same as what is plotted in Fig. 4.5(c) for the vacuum bottom. For this SSP $\beta \approx 1$ for all mode pairs where both modes are SRBR, and $\beta \approx-3$ for all mode pairs where both modes are non-SRBR. In regions where one mode is SRBR and the other is non-SRBR, $\beta$ usually greater than 1 . 
relationship to the integrated vertical phase $\phi_{1}\left(S_{p}\right)$, and can be calculated using Eq. (4.27) for adjacent modes and Eq. (4.37) for non-adjacent modes.

The relationship between $c(z), \phi_{1}\left(S_{p}\right)$, and $\beta$ provides a means for understanding how the SSP affects the value of $\beta$. A qualitative argument was given which suggests that $\beta \approx 1$ for SRBR modes in waveguides where the seafloor is modeled as a vacuum, regardless of the SSP. It was also shown that $\beta$ for non-SRBR modes can take on a wide range of values, which depend strongly on the details of the SSP.

The present analysis discussed how to calculate $\beta$ for a single pair of modes. But, as can be seen in Eq. (2.9), the acoustic intensity is a summation of terms from all mode pairs. Each term of the summation will contribute to the total intensity (although a few groups of modes sometimes dominate the summation). The relative amplitude of each term of the summation depends on the relative modal amplitudes, which in turn depend primarily on the source and receiver depths relative to features in the sound speed profile. This depth-dependence is discussed briefly in [51] and [52]. For example, if both the source and receiver are near a local sound speed minimum, the local modal amplitudes of the modes trapped in that sound speed minimum will likely be large, and one will observe some striations according to the value of $\beta$ for mode pairs with $S_{p} \approx 1 / c_{\min }$. The present analysis provides a rigorous framework for analyzing how the observed striation slopes in an acoustic intensity plot depend on the source and receiver depths and the SSP.

As with many ocean acoustic phenomena, the waveguide invariant can be understood from the perspective of normal modes or ray theory. The insights obtained by the present normal mode analysis complement the ones obtained by the ray-theoretic analysis presented in $[24,22,14]$. In fact, because ray theory can be derived by taking the WKB approximation to the limit of infinite frequency, the results from the present analysis as $\omega \rightarrow \infty$ should match the results derived in $[24,22]$. Reference [14] discusses the relationship between normal modes and ray theory in the context of the waveguide invariant.

Because the striations from the waveguide invariant can be seen in the beamformed output from a horizontal array, as will be discussed in Ch. 5, one possible application of the present analysis would be to use a horizontal array to observe striations corresponding to specific range of $k_{r}$ values, and thus a specific range of $\beta$ values. This idea is explored in Ch. 5. Knowledge of which $k_{r}$ values correspond 
to which $\beta$ values may facilitate passive range estimation and other applications of the waveguide invariant, especially in low SNR environments.

\section{A Chapter Appendix: Justification for treating $k_{r}$ as an implicit function}

$k_{r}$ can be treated as an implicit function of $n$ and $\omega$ in regions where the derivative $\partial f / \partial k_{r}$ exists and is not equal to zero [31, Sec. 7.2]. The analysis in this chapter is only interested in the region

$$
\frac{\omega}{c_{\text {bottom }}}<k_{r}<k_{\max }=\frac{\omega}{c_{\min }}
$$

where $c_{\text {bottom }}$ is the speed of sound in the bottom fluid halfspace (use $\infty$ for a vacuum), and $c_{\min }$ is the minimum sound speed in the water column. To determine when $\partial f / \partial k_{r}$ exists and is not equal to zero, use Eq. (4.12) to write:

$$
\frac{\partial f}{\partial k_{r}}=-\left(\frac{\partial \phi\left(k_{r}, \omega\right)}{\partial k_{r}}+\frac{\partial \Delta \phi_{\mathrm{dn}}}{\partial k_{r}}+\frac{\partial \Delta \phi_{\mathrm{up}}}{\partial k_{r}}\right) .
$$

Inspection of Eq. (4.4) reveals that $\partial \phi\left(k_{r}, \omega\right) / \partial k_{r}$ exists everywhere inside the region of interest except perhaps at $k_{r}$ values corresponding to discontinuities in $k(z)=\omega / c(z)$ and at $k_{r}=k(0)$ and $k_{r}=k(d) . \partial \Delta \phi_{\text {up }} / \partial k_{r}$ equals zero everywhere except at $k_{r}=k(0)$, where the derivative does not exist. $\partial \Delta \phi_{\mathrm{dn}} / \partial k_{r}$ can be calculated analytically and is smooth, continuous, and monotonically decreasing inside the region of interest.

Therefore, $k_{r}$ can be defined as an implicit function of $n$ and $\omega$ in the region of interest excluding the points $k_{r}=k(0), k_{r}=k(d), k_{r}$ values corresponding to discontinuities in $k(z)$, and possibly one more location where $\partial f / \partial k_{r}=0$.

All of the aforementioned discontinuities are artifacts of the WKB approximation used in this chapter. In a full-wave solution of the depth-separated wave equation, the effect of the surface or sea floor on the horizontal wavenumber would smoothly increase as the turning point of the mode approaches the waveguide boundary. Future research could attempt to extend the current analysis to a WKB approximation that includes the evanescent Airy tails of the non-SRBR modes. 


\section{B Chapter Appendix: Derivation of expression for $\beta$}

Equation (4.26), which includes the effects of a bottom fluid halfspace, was derived with the assistance of the computer algebra system Mathematica. As described in Sec. 4.3, when the bottom halfspace is a vacuum, Eq. (4.26) reduces to Eq. (4.27). Alternatively, Eq. (4.27) can be derived by hand if one begins the derivation assuming that the bottom halfspace is a vacuum. That derivation is performed here.

Following the WKB approximation in Sec. 4.2, for a waveguide with an arbitrary SSP and a vacuum bottom halfspace:

$$
n=\omega \cdot \phi_{1}\left(\frac{k_{r}}{\omega}\right)-1 / 2-1 / 2+1=\omega \cdot \phi_{1}\left(\frac{k_{r}}{\omega}\right) .
$$

Using the method for implicit differentiation in Sec. 4.2.2:

$$
0=f\left(k_{r}, n, \omega\right)=n-\omega \cdot \phi_{1}\left(\frac{k_{r}}{\omega}\right) .
$$

Application of Eqs. (4.13) and (4.14) yield:

$$
\begin{gathered}
\frac{\partial k_{r}}{\partial n}=\frac{1}{\phi_{1}^{\prime}\left(\frac{k_{r}}{\omega}\right)} \\
\frac{\partial k_{r}}{\partial \omega}=-\frac{\phi_{1}\left(\frac{k_{r}}{\omega}\right)-\frac{k_{r}}{\omega} \phi_{1}{ }^{\prime}\left(\frac{k_{r}}{\omega}\right)}{\phi_{1}{ }^{\prime}\left(\frac{k_{r}}{\omega}\right)} .
\end{gathered}
$$

Then apply the steps in Sec. 4.2.2 to determine the mixed-partial derivative:

$$
\begin{aligned}
\frac{\partial}{\partial \omega}\left(\frac{\partial k_{r}}{\partial n}\right) & =\frac{\partial}{\partial \omega}\left(\frac{1}{\phi_{1}^{\prime}\left(\frac{k_{r}(\omega)}{\omega}\right)}\right) \\
& =-\frac{\left(-\frac{k_{r}(\omega)}{\omega^{2}}+\frac{k_{r}{ }^{\prime}(\omega)}{\omega}\right) \phi_{1}^{\prime \prime}\left(\frac{k_{r}(\omega)}{\omega}\right)}{\phi_{1}^{\prime}\left(\frac{k_{r}(\omega)}{\omega}\right)^{2}} .
\end{aligned}
$$


Replace the $k_{r}^{\prime}(\omega)$ term in Eq .(4.44) with Eq. (4.43) and simplify the result:

$$
\begin{aligned}
\frac{\partial}{\partial \omega}\left(\frac{\partial k_{r}}{\partial n}\right) & =-\frac{\left(-\frac{k_{r}}{\omega^{2}}-\frac{\phi_{1}\left(\frac{k_{r}}{\omega}\right)-\frac{k_{r}}{\omega} \phi_{1}^{\prime}\left(\frac{k_{r}}{\omega}\right)}{\omega \phi_{1}^{\prime}\left(\frac{k_{r}}{\omega}\right)}\right) \phi_{1}^{\prime \prime}\left(\frac{k_{r}}{\omega}\right)}{\phi_{1}^{\prime}\left(\frac{k_{r}}{\omega}\right)^{2}} \\
& =\frac{\phi_{1}\left(\frac{k_{r}}{\omega}\right) \phi_{1}^{\prime \prime}\left(\frac{k_{r}}{\omega}\right)}{\omega \phi_{1}^{\prime}\left(\frac{k_{r}}{\omega}\right)^{3}} .
\end{aligned}
$$

Insert Eqs. (4.42) and (4.45) into Eq. (4.10), and write $\frac{k_{r}}{\omega}$ as $S_{p}$ :

$$
\beta\left(S_{p}\right)=-\frac{\phi_{1}^{\prime}\left(S_{p}\right)^{2}}{\phi_{1}\left(S_{p}\right) \phi_{1}^{\prime \prime}\left(S_{p}\right)}
$$

which is the same as Eq. (4.27).

For a waveguide with a fluid bottom halfspace, the expression for $n$ is that given in Eq. (4.25) instead of the one given in Eq. (4.40). The same mathematical operations are then be applied, but the intermediate expressions are lengthy and difficult to manipulate by hand. The following lines of Mathematica code were used to produce the result in Eq. (4.26).

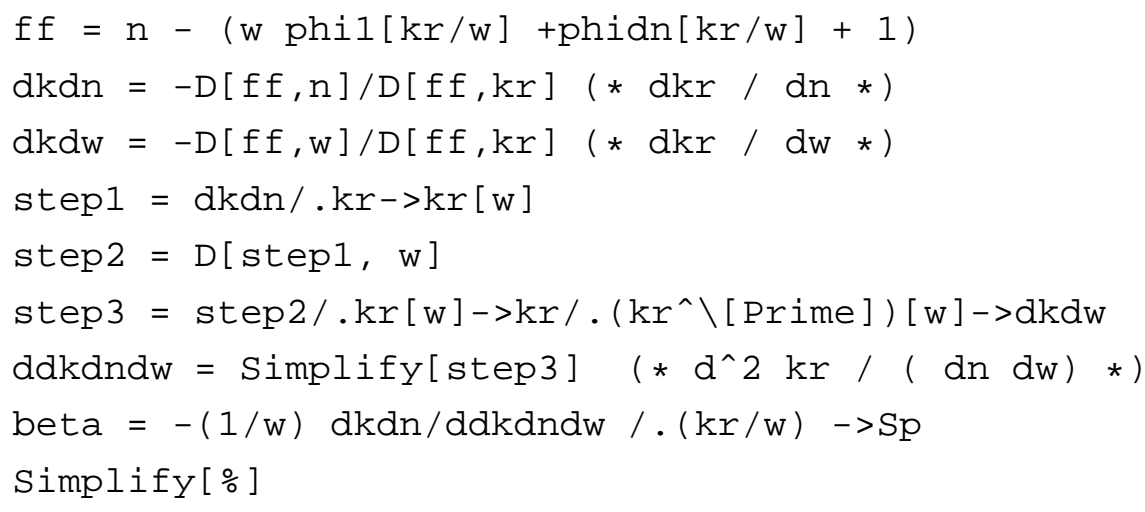

Equation (4.26) is valid when the bottom is fluid halfspace, as is the case in all of the examples in this chapter. One could extend the present analysis to include the effects of an arbitrarily layered (but lossless) bottom by letting the bottom reflection coefficient be a function of both $k_{r}$ and $\omega$ independently: $\phi_{b}\left(k_{r}, \omega\right)$. This can be done by changing the first line of the above Mathematica code to:

ff $=\mathrm{n}-(\mathrm{w} \operatorname{phil}[\mathrm{kr} / \mathrm{w}]+\operatorname{phidn}[\mathrm{kr}, \mathrm{w}]+1)$ 
The resulting expression is extremely complicated, but analytic none-the-less. 


\section{Chapter 5}

\section{Array processing with the waveguide invariant}

Chapter 3 used striations in $I(r, \omega)$ measured from a single hydrophone to estimate the range to an acoustic source, by assuming that $\beta=1$. Chapter 4 showed that surface-reflected bottom-reflected (SRBR - see Sec. 2.2.2) modes usually had $\beta_{m l}$ values close to one, whereas non-SRBR modes had $\beta_{m n}$ values that varied widely and depended strongly on the details of the SSP. In this chapter, we develop a technique for observing waveguide invariant striations with a horizontal line array (HLA), which allows for rejecting noise from sources other than the source of interest. We also show that an HLA with sufficient aperture can filter out non-SRBR modes, resulting in a striation pattern consisting only of SRBR modes which are likely to have $\beta_{m l} \approx 1$.

As with the rest of this thesis, mode numbers are denoted by $m$ and $l$.

\subsection{Introduction}

Passive source localization is one of the most straightforward applications of the waveguide invariant. If one uses a single hydrophone to measure the acoustic intensity in a shallow-water waveguide while a broadband source moves away from the hydrophone, the resulting plot of acoustic intensity will contain striations described by the waveguide invariant. The slopes of the striations can be used to estimate the 
range to the source, as was done in Ch. 3 and in [56]. This type of processing is referred to as incoherent processing because it depends only on the acoustic intensity (as opposed to the complex pressure level) and is discussed further in Sec. 5.2.

Although observing intensity striations using a single hydrophone is appealing because of its simplicity, the disadvantage is that if there are multiple sources in the water, all of those sources contribute to the striation pattern in a non-linear manner and the striations from the source of interest can become obscured. To combat this problem, one can use an array and beamform towards the source of interest. The use of an array to observe the striations necessitates coherent processing of the measured acoustic field.

In [66], Yang showed that the output of a horizontal line array (HLA) with a uniform array weights can be used to observe waveguide invariant striations. The output of the beamformer will contain striations that are almost the same as the striations present in the single-hydrophone acoustic intensity, while rejecting noise coming from directions other than that of the source of interest. In [62], Turgut et al. used the methods described in [66] to successfully localize an acoustic source with experimental data.

In Sec. 5.3, it is shown that when the HLA is short and the temporal frequency is low (small aperture relative to wavelength), processing the array data with a uniformly weighted beamformer will yield the expected results: the array processor output will contain desired striations from the source of interest while rejecting noise from other directions. But when the array is long or the temporal frequency is high (large aperture relative to wavelength), uniformly weighted beamforming can have an undesired effect: the main lobe of a uniformly weighted beamformer can get so narrow that it eliminates some of the modes necessary to create the modal interference pattern (striations) that is one is trying to observe.

This chapter shows how to design HLA array weights that are optimal for observing waveguide invariant striations from a single source while rejecting noise from other directions. This chapter also analyzes when it is beneficial to design such array weights as opposed to simply using uniform ones.

Section 5.4 uses simulated data to illustrate how an HLA can filter out nonSRBR modes which typically don't have $\beta_{m l} \approx 1$. Sec. 5.5 uses experimental data (from the same experiment that was used in Ch. 3) to show that an HLA can 
reject noise but preserve the waveguide invariant striations. Sec. 5.6 summarizes and concludes the chapter.

Before moving forward with the present analysis, it is worth noting that other array processing techniques have been developed to exploit the waveguide invariant, including source localization using broadband matched field processing [59], and covariance matrix estimation for adaptive beamforming [57, 37] (See Appendix A). In the present analysis, the focus is on using an HLA to filter out non-SRBR modes, and/or observe the waveguide invariant striations from one source while eliminating contributions to the acoustic intensity from other sources.

\subsection{Review of incoherent processing}

As discussed in Sec. 2.3.1, the acoustic intensity in a waveguide resulting from a single, compact acoustic source can be written as summation of cosines [see Eq. (2.10)]. Many processing schemes that utilize the waveguide invariant, such as the one presented in Ch. 3, rely on accurately observing the striations resulting from the cosine terms in Eq. (2.10). But when other sound sources are present in the ocean, the striations from the source of interest can be become difficult to observe amongst all the noise. An example of this can be seen in Fig. 5.4(a).

If one has an array of hydrophones rather than a single hydrophone, the striations from the source of interest can be enhanced by using coherent processing. But doing so requires a judicious choice of array weights, as will be discussed in the next section.

\subsection{Coherent processing}

The concept of observing waveguide invariant striations using an HLA was originally introduced by Yang in [66], which analyzed the special case of an HLA with uniform array weights. In this section we generalize Yang's results to array processors (beamformers) with non-uniform weights, such as a Hamming Window or weights designed by more advanced techniques. We restrict our attention to uniformly spaced HLAs. It will be shown that the trade-off to be considered when choosing the weights is not the usual "beam width versus side lobe level" that one 
encounters with planewave array processing, because the desired signal is not a planewave but rather the striations resulting from the interference between a set of modes.

We start by defining some notation (bold faced lower-case letters denote vector quantities):

- $c_{0}$ is the speed of sound at the HLA depth.

- $k_{0}=\frac{\omega}{c_{0}}$ is the wavenumber at the HLA depth.

- $J$ is the number of elements in the HLA, with each element numbered as $j=1, j=2, \ldots, j=J$.

- $\theta_{L}$ is the look direction as measured from broadside of the HLA.

- $k_{L}=k_{0} \sin \theta_{L}$ is the horizontal wavenumber corresponding to the look direction.

- $\mathbf{v}\left(k_{L}\right)=\left[v_{1}\left(k_{L}\right), v_{2}\left(k_{L}\right), \cdots, v_{J}\left(k_{L}\right)\right]^{T}$ is the steering vector corresponding to the look direction. $\mathbf{v}$ is a column vector.

- $\mathbf{w}=\left[w_{1}, w_{2}, \cdots, w_{J}\right]^{T}$ the array weights column vector (e.g., Hamming, Hann, uniform). w is a real-valued vector and does not include the phase shift of the steering vector.

- $\theta_{S}$ is the bearing of the acoustic source as measured from broadside of the HLA, in the plane parallel to the ocean surface.

- $\Upsilon$ is the wavenumber response function of the array processor, which depends on the array geometry and the array weights $\mathbf{w}$, and is described in more detail later.

\subsubsection{HLA response to a planewave}

An array and its weights can be characterized by their wavenumber response function, denoted here by $\Upsilon$ [61, Chs. 2 and 3]. $\Upsilon$ describes the gain of an array processor for a planewave with a given wavenumber along the axis of the array. As shown in [61, Eq. 2.122], $\Upsilon$ for a uniformly spaced HLA depends only on the difference 
between wavenumber corresponding to look direction $\left(k_{L}\right)$ and the wavenumber corresponding to the source direction $\left(k_{S}\right)$, as opposed to depending on $k_{L}$ and $k_{S}$ independently. $\Upsilon$ also depends on (is parameterized by) the array weights. Thus we write the wavenumber response function as $\Upsilon\left(k_{L}-k_{S} ; \mathbf{w}\right)$. It is well known that when $\mathbf{w}=1, \Upsilon\left(k_{L}-k_{S} ; \mathbf{1}\right)$ is a periodic sinc function of $\left(k_{L}-k_{S}\right)$ [61, Eq. 2.94].

Let a superscript $H$ represent a complex conjugate transpose, and an asterisk represent the complex conjugate of a scalar. The wavenumber response function is then defined as [61, Eq. 2.58]

$$
\Upsilon\left(k_{L}-k_{S} ; \mathbf{w}\right) \equiv \mathbf{v}\left(k_{S}\right)^{H}\left(\mathbf{v}\left(k_{L}\right) \mathbf{w}\right)=\sum_{j=1}^{J} v_{j}\left(k_{S}\right)^{*}\left(v_{j}\left(k_{L}\right) w_{j}\right)
$$

where $\left(\mathbf{v}\left(k_{L}\right) \mathbf{w}\right)$ is the element-by-element multiplication of $\mathbf{v}\left(k_{L}\right)$ and $\mathbf{w}$.

For an HLA with $\mathrm{J}$ elements numbered 1 to $\mathrm{J}$ and an inter-element spacing of $d$, the $j$-th element is located at a distance of $d(j-1)$ from the 1 st element, and the center of the array is at a distance of $d(J-1) / 2$ from the 1 st element. Let $d_{j}$ represent the displacement of the $j$-th element of the array to the center of the array:

$$
d_{j}=d(j-1)-d(J-1) / 2
$$

The $j$-th element of the steering vector $\mathbf{v}$ is then (Eq. 2.78 of Van Trees)

$$
v_{j}\left(k_{L}\right)=\exp \left[+i k_{L} d_{j}\right]
$$

In this chapter we only consider $\mathrm{w}$ that are real and symmetric with respect to the center of the array. Because of that, and because $d_{j}$ was defined with respect to the center of the array, $\Upsilon\left(k_{L}-k_{S} ; \mathbf{w}\right)$ will be real. This is a result of the Discrete Fourier Transform relationship between $\Upsilon$ and $\mathbf{w}$ [61, Sec. 2.3]. Knowing that $\Upsilon\left(k_{L}-k_{S} ; \mathbf{w}\right)$ is real will help simplify the algebra later in this chapter, but is of little conceptual importance. 


\subsubsection{HLA response to source in waveguide}

$\Upsilon\left(k_{L}-k_{S} ; \mathbf{w}\right)$ represents the response of an array to the pressure field of a planewave. In this subsection we derive the response of an HLA array with weights $\mathbf{w}$ to the pressure field from a discrete source in a waveguide. The intensity of that response, when plotted as a function of range and frequency, will contain waveguide invariant striations. [66] contains a similar derivation for the specific case of uniform array weights $(\mathbf{w}=\mathbf{1})$. In the present work, we generalize Yang's result and explain some advantages of using non-uniform array weights.

Let $\Upsilon_{\text {total }}\left(\omega, \theta_{L}, \theta_{S}, r_{c} ; \mathbf{w}\right)$ represent the response function of an HLA due to a source at a bearing of $\theta_{S}$ and a range of $r_{c}$ in a range independent waveguide, while looking in a direction $\theta_{L}$. In contrast to $\Upsilon$ which depends only on $k_{L}-k_{S}, \Upsilon_{\text {total }}$ depends on $\omega, \theta_{L}$ and $\theta_{S}$ for reasons that will become clear later; it also depends on the array weights $\mathbf{w}$. It will be shown that $\Upsilon_{\text {total }}$ can be written as a sum of $\Upsilon_{\mathrm{s}}$, one for each mode.

$$
\Upsilon_{\text {total }}\left(\omega, \theta_{L}, \theta_{S}, r_{c} ; \mathbf{w}\right) \equiv \mathbf{p}^{H}\left(\mathbf{v}\left(k_{L}\right) \mathbf{w}\right)
$$

where $\mathbf{p}$ is the pressure at the array elements due to the source in the waveguide. Note that $k_{L}$ is a function of $\theta_{L}$, as defined at the beginning of this section.

To calculate $\mathbf{p}$, let $r_{c}$ be the range from the source to the center of the HLA. If the distance between the array and the source is large compare to the array's length, then the range from the source to the $j$-th element of the array can be approximated as

$$
r_{c}+\sin \theta_{S} d_{j}
$$

The pressure at the $j$-th element of the array can written by replacing the $r$ in Eq. 2.7 with Eq. 5.5, resulting in:

$$
p_{j}=\sum_{m} A_{m} \exp \left[+i k_{r m}\left(r_{c}+\sin \theta_{S} d_{j}\right)\right]
$$

Eq. (5.6) can be interpreted as a sum of planewaves along the array, each with 
amplitude an $A_{m}$, a wavenumber along the array of $k_{r m} \sin \theta_{S}$, and a phase of $k_{r m} r_{c}$.

Inserting Eqs. (5.6) and (5.3) into Eq. (5.4), and rearranging the summations yields:

$$
\begin{aligned}
\Upsilon_{\text {total }}\left(\omega, \theta_{L}, \theta_{S}, r_{c} ; \mathbf{w}\right)=\mathbf{p}^{H} \mathbf{v}\left(k_{L}\right) \mathbf{w} \\
=\sum_{j=1}^{J} p_{j}^{*} v_{j}\left(k_{L}\right) w_{j} \\
=\sum_{j=1}^{J}\left(\sum_{m} A_{m} \exp \left[+i k_{r m}\left(r_{c}+\sin \theta_{S} d_{j}\right)\right]\right)^{*} \exp \left[+i k_{L} d_{j}\right] w_{j} \\
=\sum_{m} A_{m} \exp \left[-i k_{r m} r_{c}\right] \sum_{j=1}^{J} \exp \left[-i k_{r m} \sin \theta_{S} d_{j}\right] \exp \left[+i k_{L} d_{j}\right] w_{j}
\end{aligned}
$$

The summation over $j$ in Eq. (5.8) is the same as Eq. (5.1), but with the $k_{S}$ in Eq. (5.1) replaced by $k_{r m} \sin \theta_{S}$. Eq. (5.1) is the definition of $\Upsilon$ for a planewave. We can therefore write $\Upsilon_{\text {total }}$ as a sum of $\Upsilon_{s}$ - one for each mode $m$.

Define $k_{m S} \equiv k_{r m} \sin \theta_{S}$, which is the wavenumber of mode $m$ along the array due to a source at a bearing $\theta_{S}$. $\Upsilon_{\text {total }}$ can then be written as

$$
\Upsilon_{\text {total }}\left(\omega, \theta_{L}, \theta_{S} ; \mathbf{w}\right)=\sum_{m} A_{m} \exp \left[-i k_{r m} r_{c}\right] \Upsilon\left(\omega, k_{L}-k_{m S}\right)
$$

bearing in mind that $k_{L}$ is a function of $\theta_{L}$, and $k_{m S}$ is a function of $\theta_{S}$. To the HLA, each mode appears to be a planewave propagating along the array with a wavenumber of $k_{m S}$, with an amplitude of $A_{m}$, and a phase shift of $k_{r m} r_{c}$. So $\Upsilon_{\text {total }}$ is a sum of $\Upsilon$ s corresponding to each mode.

The magnitude of $\Upsilon_{\text {total }}\left(\omega, \theta_{L}, \theta_{S} ; \mathbf{w}\right)$ contains the waveguide invariant stria- 
tions analogous to those in Eq. (2.10):

$$
\begin{aligned}
\left|\Upsilon_{\text {total }}\left(\omega, \theta_{L}, \theta_{S} ; \mathbf{w}\right)\right|^{2}=\left|\sum_{m} A_{m} \exp \left[-i k_{r m} r_{c}\right] \Upsilon\left(\omega, k_{L}-k_{m S} ; \mathbf{w}\right)\right|^{2} \\
=\sum_{q} A_{q}^{2}\left|\Upsilon\left(k_{L}-k_{q S} ; \mathbf{w}\right)\right|^{2} \\
\quad+\sum_{m, l ; m \neq l} A_{m} A_{l} \cos \left(\Delta k_{m l} r_{c}\right)\left(\Upsilon\left(k_{L}-k_{m S} ; \mathbf{w}\right)\right)\left(\Upsilon\left(k_{L}-k_{l S} ; \mathbf{w}\right)\right)
\end{aligned}
$$

where $\Delta k_{m l}=k_{r m}-k_{r l}$ is the difference in the horizontal wavenumbers between mode $m$ and mode $l$, and we have used the fact that $\Upsilon\left(k_{L}-k_{m S} ; \mathbf{w}\right)$ is real.

A careful term-by-term comparison of Eq. (5.10) to Eq. (2.10) is essential to understanding the present analysis. The waveguide invariant striations are a result of the $\cos \left(\Delta k_{m l} r_{c}\right)$ terms, which represent the interference between modes. The cosine terms in Eq. (5.10) are the same as in Eq. (2.10) but are multiplied by $\left(\Upsilon\left(k_{L}-k_{m S} ; \mathbf{w}\right)\right)\left(\Upsilon\left(k_{L}-k_{l S} ; \mathbf{w}\right)\right)$.

In order to "extract" the waveguide invariant striations from HLA measurements of the acoustic field, one would first compute the complex pressure level at each hydrophone of the array at each snapshot. The resulting complex pressure levels would then be fed into an array processor, and the magnitude of the output of the array processor would be plotted versus frequency and snapshot number.

For the purpose of understanding the present work, it is easiest to consider a scenario where the source changes range but not bearing between snapshots so that one can plot $\left|\Upsilon_{\text {total }}\left(\omega, \theta_{L}, \theta_{S} ; \mathbf{w}\right)\right|^{2}$ versus $r_{c}$ and $\omega$ while keeping $\theta_{L}$ fixed at

$\theta_{S}$. (In a practical situation, one would likely plot $\left|\Upsilon_{\text {total }}\left(\omega, \theta_{L}, \theta_{S} ; \mathbf{w}\right)\right|^{2}$ versus snapshot number and $\omega$ while changing $\theta_{L}$ with the snapshot number to follow the source, and then estimate the range and speed of the source. See [62].)

\subsubsection{Array processing for the waveguide invariant}

To simplify notation, let $\Delta k$ equal the difference between a wavenumber received by an array, and the wavenumber corresponding to the look direction of array processor. That is, $\Delta k=k_{L}-k_{S}$ in free space or $\Delta k=k_{L}-k_{m S}$ in a waveguide. 
The purpose of array processing is to exclude contributions to the output of the array processor from sources located at a bearings different than that of the source of interest. Traditionally, array processing (beamforming) was developed to distinguish planewaves from one another, and thus the goal was the design array weights that resulted in as narrow of a main lobe in $\Upsilon$ as is possible while controlling the level of the side lobes in $\Upsilon$ [61, Ch. 3]. The array weights were usually chosen so that $\Upsilon(\Delta k ; \mathbf{w})$ was largest at $\Delta k=0$ and $\Upsilon(\Delta k ; \mathbf{w})$ generally decreased in value as $\Delta k$ increased.

In contrast to the planewave model, the waveguide invariant striations are the result of modes interfering with one another. In the previous subsection it was shown that each mode appears to the HLA as a planewave with wavenumber $k_{m S}$. If the array processor weights have a high enough wavenumber resolution (i.e., main lobe width) to distinguish a low order mode (e.g., $k_{1 S}$ ) from a high order model (e.g., $k_{8 S}$ ) coming from the same source, then the striations resulting from the interference term between the two modes, $\cos \left(\Delta k_{m l} r_{c}\right)\left(\Upsilon\left(k_{L}-\right.\right.$ $\left.\left.k_{m S} ; \mathbf{w}\right)\right)\left(\Upsilon\left(k_{L}-k_{l S} ; \mathbf{w}\right)\right)$ from Eq. (5.10), will have a very small magnitude because either $\left(\Upsilon\left(k_{L}-k_{m S} ; \mathbf{w}\right)\right)$ or $\left(\Upsilon\left(k_{L}-k_{l S} ; \mathbf{w}\right)\right)$ will be small no matter what the value of $k_{L}$ is. That is, no matter where the array is steered, the desired striations term may be suppressed due to the high resolution of the array processor. We investigate this further in the next subsection, and then suggest how to design better array weights later in this chapter.

\subsubsection{Uniform weighs}

This subsection analyzes the circumstances which result in suppressed striations for the case of an array processor with uniform weights. [66] derived $\Upsilon_{\text {total }}\left(\omega, \theta_{L}, \theta_{S} ; \mathbf{w}\right)$ for the specific case of $\mathbf{w}=1$. In that case, $\Upsilon$ is a periodic sinc function [61, Eq. 2.94]:

$$
\Upsilon(\Delta k ; \mathbf{w})=\frac{\sin \left(\frac{J d}{2} \Delta k\right)}{\sin \left(\frac{d}{2} \Delta k\right)}
$$


Inserting this into Eq.(5.10) yields

$$
\begin{aligned}
= & \sum_{q} A_{q}^{2}\left|\frac{\sin \left(\frac{J d}{2}\left(k_{L}-k_{q S}\right)\right)}{\sin \left(\frac{d}{2}\left(k_{L}-k_{q S}\right)\right)}\right|^{2} \\
& +\sum_{m, l ; m \neq l} A_{m} A_{l} \cos \left(\Delta k_{m l} r_{c}\right)\left(\frac{\sin \left(\frac{J d}{2}\left(k_{L}-k_{m S}\right)\right)}{\sin \left(\frac{d}{2}\left(k_{L}-k_{m S}\right)\right)}\right)\left(\frac{\sin \left(\frac{J d}{2}\left(k_{L}-k_{l S}\right)\right)}{\sin \left(\frac{d}{2}\left(k_{L}-k_{l S}\right)\right)}\right)
\end{aligned}
$$

Eq. (5.12) is equivalent to [66, Eqs. 14 and 15]. The second term of Eq. (5.12) contains the same cosine terms that are in Eq. (2.10), but they are multiplied by two periodic sinc functions.

When $(|J d / 2 \Delta k| \ll \pi)$, the periodic sinc function is near its maximum value. As $|J d / 2 \Delta k|$ increases in value, the periodic sinc function decreases in value until $|J d / 2 \Delta k|>\pi$, at which point the periodic sinc function will have a very small value (i.e., be in the side lobe of the periodic sinc).

$(J d / 2)$ is approximately half of the array length. So if the array length times $\left(k_{l S}-k_{m S}\right)$ differ by an amount larger than twice the main lobe width of $\Upsilon(\Delta k ; \mathbf{w})$, then the cosine term in Eq. (5.12) is going to be very small no matter what the value of $k_{L}$ is.

This can occur in practical situations. Consider a waveguide where the water column has a sound speed of $1500 \mathrm{~m} / \mathrm{s}$ and the seabed has a sound speed of 1600 $\mathrm{m} / \mathrm{s}$. If a source with a center frequency of $500 \mathrm{~Hz}$ is at end fire, then: $k_{m S}=k_{r m}$, the first order mode has $k_{r 1} \approx 2 \pi 500 / 1500$, and highest order mode has $k_{r M} \approx$ $2 \pi 500 / 1600$. An array that is longer than about 200 meters (with $\mathbf{w}=1$ ) will have such a narrow main lobe that there is no choice of $k_{L}$ for which $\Upsilon$ for mode 1

$$
\frac{\sin \left(\frac{J d}{2}\left(k_{L}-k_{r 1}\right)\right)}{\sin \left(\frac{d}{2}\left(k_{L}-k_{r 1}\right)\right)}
$$

and $\Upsilon$ for mode $M$

$$
\frac{\sin \left(\frac{J d}{2}\left(k_{L}-k_{r M}\right)\right)}{\sin \left(\frac{d}{2}\left(k_{L}-k_{r M}\right)\right)}
$$

are in their respective main lobes.

Although one may not always want the term corresponding to $\Delta k_{1 M}$ to be in the array output, an analogous argument can be made for any two modes with 
differing horizontal wavenumbers. Even if the two modes are both in the main lobe, their cosine term will be suppressed to some extent because the periodic sinc function starts to decrease in value as soon as the wavenumber difference in the argument isn't zero.

In this subsection, we showed why uniform weights $(\mathbf{w}=\mathbf{1})$ can have undesired results. In the next subsection, we show how to choose the weights to prevent the suppression of the desired cosine terms.

In addition to the issue analyzed in this section regarding the suppression of cosine terms in the summation, there is one other potential problem with the array processor output. As noted in [66], $\left(k_{L}-k_{m S}\right)$ and $\left(k_{L}-k_{l S}\right)$ are functions of $\omega$. And so multiplying the cosine term with

$$
\frac{\sin \left(\frac{J d}{2}\left(k_{L}-k_{m S}\right)\right)}{\sin \left(\frac{d}{2}\left(k_{L}-k_{m S}\right)\right)} \frac{\sin \left(\frac{J d}{2}\left(k_{L}-k_{l S}\right)\right)}{\sin \left(\frac{d}{2}\left(k_{L}-k_{l S}\right)\right)}
$$

may have an adverse affect on the striation pattern, which lies in the $\left(r_{c}, \omega\right)$ plane. It will become clear in the next subsection that designing a $w$ that prevents the suppression of the cosine terms will also prevent $\Upsilon\left(k_{L}-k_{m S} ; \mathbf{w}\right)$ and $\Upsilon\left(k_{L}-\right.$ $\left.k_{l S} ; \mathbf{w}\right)$ from being a strong function of frequency and thus preserve the desired striations resulting from the cosine terms.

\subsubsection{Designing array weights for waveguide invariant striations}

The previous subsection discussed why uniform weights can lead to a suppression of some striation terms because uniform weights correspond to a narrow main lobe in $\Upsilon$. In this subsection we discuss how to choose non-uniform weights for the purpose of observing the waveguide invariant striations. To understand this subsection, it is useful to think of the array processor as a spatial filter whose wavenumber response is $H(k)$ where $k$ is the wavenumber along the array.

In the case of planewave beamforming - where the goal is to estimate the signal coming from a one direction while rejecting signal coming from other directions - the ideal $H(k)$ is an impulse in $k$ space. Because that cannot be achieved with a finite aperture array, one chooses a trade-off main lobe width and side lobe levels. 
But in the case of using an array to observe the waveguide invariant striations, the ideal $H(k)$ is not an impulse in $k$ space; the interference pattern is the result of modes with different $k$ values interfering with each other, and so a range of $k$ values must pass through the array processor.

An ideal $H(k)$ for observing the waveguide invariant striations is an $H(k)$ that passes through all wavenumbers associated with the cosine terms in Eq.(5.10) while blocking out all other wavenumbers, so that none of the striation terms are suppressed but noise coming from directions other than the source direction get blocked out (other ideal $H(k)$ s are discussed later in this subsection). For example, in a Pekeris waveguide with $c_{\text {water }}=1500 \mathrm{~m} / \mathrm{s}$ and $c_{\text {bottom }}=1600 \mathrm{~m} / \mathrm{s} H(k)$, all modal horizontal wavenumbers will be between $\frac{\omega}{1500}$ and $\frac{\omega}{1600}$. So the array processor should pass all wavenumbers between $k=\sin \theta_{S}\left(\frac{\omega}{1600}\right)$ and $k=\sin \theta_{S}\left(\frac{\omega}{1500}\right)$ with equal amplitudes while blocking signals with all other wavenumbers:

$$
H_{\text {ideal }}(k)= \begin{cases}0 & k<\sin \theta_{S}\left(\frac{\omega}{1600}\right) \equiv k_{\text {lower }} \\ 1 & \sin \theta_{S}\left(\frac{\omega}{1600}\right) \leq k \leq \sin \theta_{S}\left(\frac{\omega}{1500}\right) \\ 0 & k>\sin \theta_{S}\left(\frac{\omega}{1500}\right) \equiv k_{\text {upper }}\end{cases}
$$

$H_{\text {ideal }}(k)$ in Eq. (5.16) is a complex band-pass spatial filter whose spatial cutoff wavenumbers depend on the temporal frequency $\omega$ and the source direction $\theta_{S}$. It is complex in the sense that the input signal is complex (the complex pressure level along the array at some value of $\omega$ ). So the filter must treat positive and negative wavenumbers differently, in contrast to typical time domain band-pass filters whose impulse response is generally conjugate symmetric (which treat positive and negative frequencies the same when the input signal is real).

$H_{\text {ideal }}(k)$ can only be realized with a infinitely long array, so it is necessary to approximate $H_{\text {ideal }}(k)$. This can be done with a finite impulse response (FIR) filter of length $J$ because there are $J$ elements in the array. A straightforward approach to designing the FIR filter is to first design a linear-phase, low-pass FIR filter centered at $k=0$ using standard digital filter design techniques [46, Ch. 7]. The resulting FIR filter will be the array weights $\mathbf{w}$. (Note that a linear phase filter is required because it yields a symmetric $\mathbf{w})$. Then multiply $\mathbf{w}$ by the steering vector $\mathbf{v}$ to shift the pass-band from being centered at $k=0$ to being centered at some $k$ value that 
is halfway between the $k_{\text {lower }}$ and $k_{\text {upper }}$ in Eq. (5.16).

For example, in the above case, one would first design a low-pass filter with a cutoff frequency of $k_{\text {cutoff }}=\left(k_{\text {upper }}-k_{\text {lower }}\right) / 2$. One would then multiply that filter by $\mathbf{v}\left(k_{\text {center }}\right)$ to shift the center frequency from up to $k_{\text {center }}=\left(k_{\text {upper }}+k_{\text {lower }}\right) / 2$. The resulting output can then be described by Eq. (5.10) where the $k_{L}=k_{\text {center }}$. Designing $\mathbf{w}$ involves choosing between many trade-offs, which are discussed in digital filter design text books such as [46]. The next section discusses one possible choice of $\mathbf{w}$ in depth.

$H_{\text {ideal }}(k)$ depends on the $\theta_{S}$ and the sound speeds in the environment, which may not be known accurately. In that case, the pass-band of the filter can be widened at the expense of letting in more noise. Or one could narrow the passband in order to reject more noise, at the expense of suppressing some of the cosine terms.

In some cases, one may purposely choose a filter that is narrower than the $H_{\text {ideal }}(k)$ in Eq. (5.16). The waveguide invariant striations (cosine terms) are most useful when the value of $\beta_{m l}$ is predictable (i.e., not sensitive to the details of the environment). But $\beta_{m l}$ is only predictable under certain circumstances: Sec. 2.3 and Ch. 4 qualitatively showed that when both modes are SRBR modes that are not near cutoff, $\beta_{m l} \approx 1$. For other modes, the value of $\beta$ is often unpredictable. Therefore, it may be useful to filter out wavenumbers that correspond to modes close to cutoff, and to filter out wavenumbers that correspond to non-SRBR modes. Then the $\cos \left(\Delta k_{m l} r\right)$ terms with unpredictable $\beta_{m l}$ values will be suppressed and the array processor output may be more useful.

It is worth noting that filtering out wavenumbers corresponding to non-SRBR modes while keeping those corresponding to SRBR modes requires fairly high wavenumber resolution, and thus requires a long array relative to the wavenumber differences.

\subsection{A simulated example}

To validate the theory presented in the previous section and explain some of the details required for implementation, a simulated example is provided here. The environment and array parameters are chosen specifically to illustrate the concepts 
from the previous section, but are realistic none-the-less.

The environment is a range-independent shallow-water waveguide with a typical summer sound speed profile (SSP), shown in Fig. 5.1. The bottom is a halfspace with sound speed of $1600 \mathrm{~m} / \mathrm{s}$, a density of $1.5 \mathrm{~g} / \mathrm{cm}^{3}$, and a attenuation of $1 / 2 \mathrm{~dB} / \lambda$. The array was a 256 element HLA with a spacing corresponding to $\lambda / 2$ at $700 \mathrm{~Hz}$; the total array length was 273 meters. The source was at endfire, which maximizes the effective aperture of the array.

The acoustic field was calculated using Kraken [48] from a range of $3000 \mathrm{~m}$ to $5000 \mathrm{~m}$, and a temporal frequency of $300 \mathrm{~Hz}$ to $700 \mathrm{~Hz}$. The source and receiver depth (52 and 84 meters, respectively) were chosen to be below the thermocline so that the bottom-trapped modes would contribute to the total pressure.

\subsubsection{Filter design}

As discussed in Sec. 5.3.5, it can be useful to filter out modes that do not interact with the top and bottom of the waveguide, because those modes are unlikely not lead to a $\beta_{m l}$ value that is close to one. Modes that are close to cutoff will also not have $\beta_{m l} \approx 1$, but modes that are close to cutoff have very small amplitudes because they are highly attenuated, so it is usually not necessary to filter them out. Therefore the ideal array processor for this environment would filter all wavenumbers except those with phase speeds between $1535 \mathrm{~m} / \mathrm{s}$ and $1600 \mathrm{~m} / \mathrm{s}$, because modes with those phase speeds are top and bottom interacting modes.

To create an linear-phase FIR filter that approximates the ideal filter, a technique that minimizes the integrated squared error between the ideal filter and the magnitude response of the filter was used (specifically, the firls tool in MAT$\mathrm{LAB}$ ). Because the wavenumber associated with a specific phase speed is a function of frequency (e.g., $k=\frac{\omega}{1600}$ ), a different FIR filter must be designed for each

temporal frequency. The width of the pass-band is $\Delta k=\omega\left(\frac{1}{1535}-\frac{1}{1600}\right)$, so at higher frequencies the FIR filter must have a wider pass-band.

\subsubsection{Simulated results}

Figure 5.2 shows the response of the FIR filters (array processor weights) designed for (a) $f=300 \mathrm{~Hz}$, (b) $f=500 \mathrm{~Hz}$, and (c) and $f=700 \mathrm{~Hz}$. Also shown is the 
response of uniform array weights, which is the same for all temporal frequencies. In subfigure (a) $(f=300 \mathrm{~Hz})$, the FIR filter response is similar to the uniform weights, but slightly wider. In subfigure (c) $(f=700 \mathrm{~Hz})$, the difference between the FIR filter and the uniform weights is very apparent; the FIR filter has a wide, flat, pass-band that allows all the desired modes the pass through.

As discussed in Sec. 2.3, waveguide invariant striations are of the form

$$
\frac{\delta f}{\delta r}=\beta \frac{f}{r}
$$

where $\frac{\delta f}{\delta r}$ is the slope of the striation and $\beta$ is the waveguide invariant. So striations with $\beta=1$ correspond to lines of the form $f=C_{1} r$ where $C_{1}$ is an arbitrary constant. Figure 5.3 shows the intensity from the (a) unfiltered pressure and (b) array processed (filtered) pressure. The black lines correspond to $\beta=1$. Notice that the striations in subfigure (b) correspond much more closely to $\beta=1$ than do the striations in subfigure (a), because the pressure from which the intensity in subfigure (b) was calculated was filtered (array processed) to remove modes that were not top and bottom interacting.

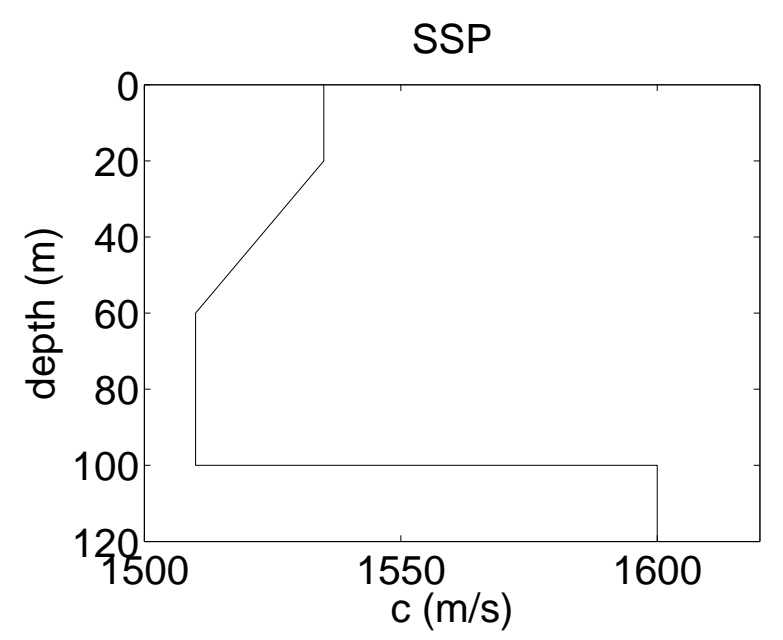

Figure 5.1: The sound speed profile for the simulated environment. It is a simplified model of a shallow-water waveguide in the summer. The bottom halfspace starts at 100 meters. 
(a) $(f=300 \mathrm{~Hz})$

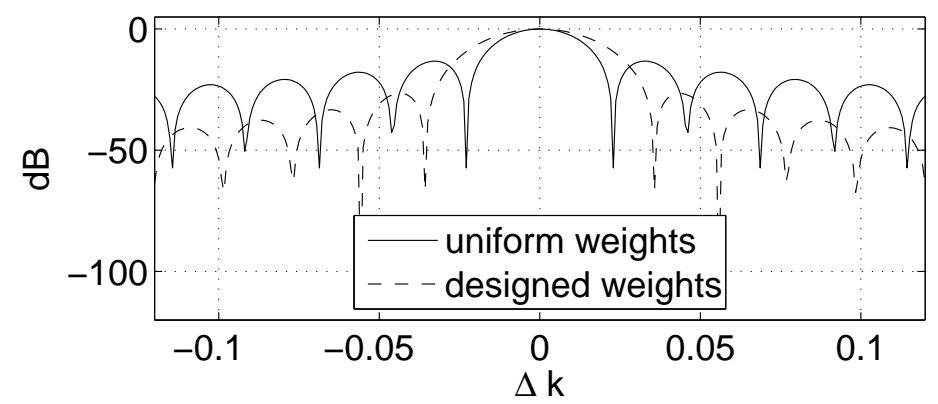

(b) $(f=500 \mathrm{~Hz})$

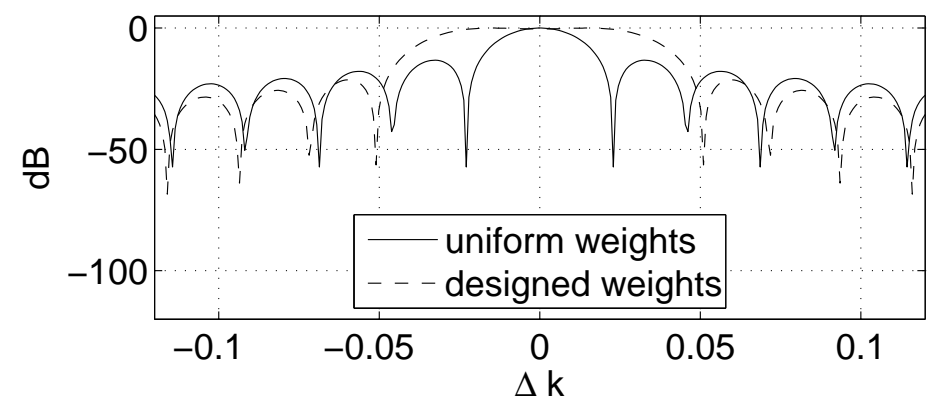

(c) $(f=700 \mathrm{~Hz})$

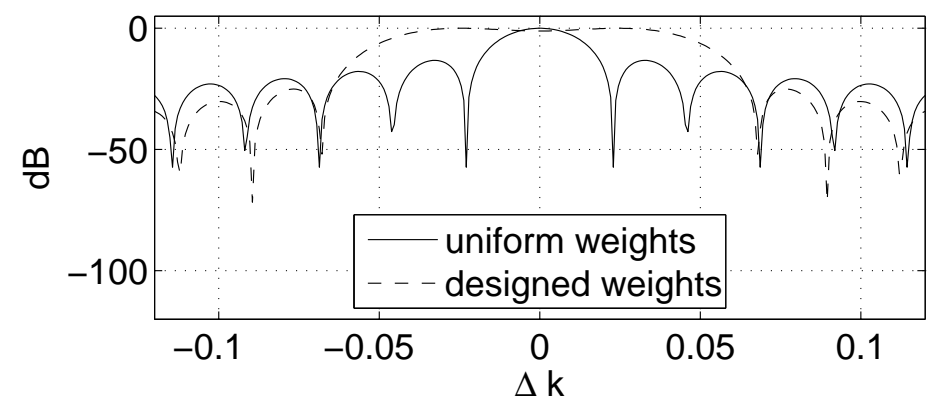

Figure 5.2: Plots of the wavenumber response function of three different array weights, each designed for a different temporal frequency $f$. The response due to uniform array weights is plotted in each subfigure as well, for comparison. (a) $f=300 \mathrm{~Hz}$. (b) $f=500 \mathrm{~Hz}$. (c) $f=700 \mathrm{~Hz}$. As $f$ increases, the array weights change so that the main lobe width increases to ensure that all the desired modes pass through the array processor without being attenuated. In contrast to the array weights designed specifically for observing the waveguide invariant, the uniform array weights do not change with $f$ and thus their response function is the same for all $f$. 
(a) unfiltered

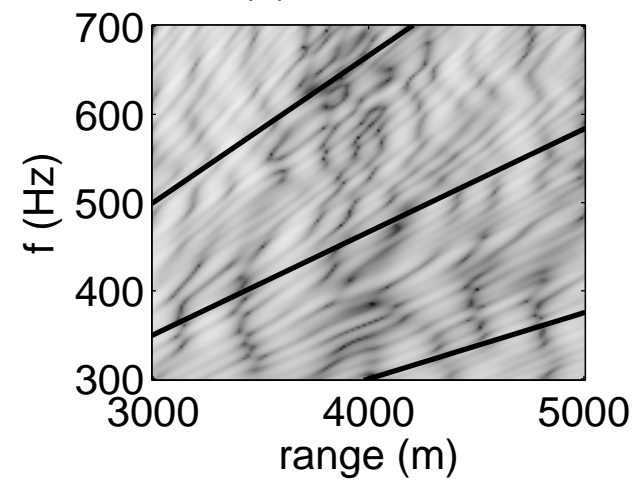

(b) filtered

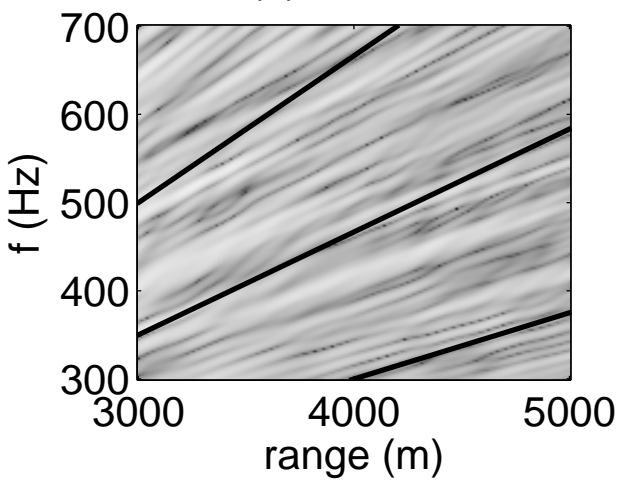

Figure 5.3: Simulated acoustic intensity as a function of range and frequency, which exhibits striations described by the waveguide invariant $\beta$. Subfigure (a) is a plot of the intensity from the scalar pressure. Subfigure (b) is a plot of the intensity from the array-processed pressure which had wavenumbers corresponding to undesired modes filtered out. The black lines correspond to $\beta=1$. The striations subfigure (b) correspond much more closely to $\beta=1$ than do the striations in subfigure (a), because the undesired modes have been filtered out.

\subsection{Experimental example}

The previous section showed an example of how filter out specific wavenumbers due to a single source. It required a relatively long array in order to have high enough wavenumber resolution. In this section, some experimental results are presented using a much shorter array. With a short array, the array weights designed using the technique from the previous section will be very similar to uniform array weights, because the desired resolution will be close to the highest resolution the array is capable of, which is achieved with uniform array weights. None-the-less, the array processing is very useful at eliminating sound coming from directions other than that of the source of interest.

The data set presented here is the same experimental data that was used for Ch. 3. In Ch. 3, only the signal from a single hydrophone was processed. But during the experiment, data was collected from a horizontal array of hydrophones. So we now process the same data using the array processing techniques discussed in this chapter. 
An acoustic source was lowered to a depth of 40 meters, and pseudorandom noise was projected from the source over a frequency band of 350 to $700 \mathrm{~Hz}$. A 23element, $17.25 \mathrm{~m}$ long hydrophone array was towed directly away from the source along a line of nearly constant bathymetry at a speed of $1.5 \mathrm{~m} / \mathrm{s}$. The hydrophone array had an inter-element spacing of $3 / 4 \mathrm{~m}$, and was towed at a depth of $50 \mathrm{~m}$. The sea floor was about $90 \mathrm{~m}$ deep with a typical summer downward refracting sound speed profile (SSP). (See Sec. 3.4.2 for more details).

Fig. 5.4 (a) shows a plot of the acoustic intensity as measured by a single hydrophone from the array, and is the exact same data as that shown in Fig. 3.8. Because $\beta \approx 1$ for the environment where the data was collected [19], one expects to see waveguide invariant striations from the acoustic source of interest that point toward the origin of the coordinate system (towards the lower left of each subfigure). Although such striations are clearly present at frequencies of 350 to $700 \mathrm{~Hz}$, there is also noise present. One particularly loud noise source causes its own parabolic shaped striations at all frequencies. Figure 5.4 (b) shows the output of the array processor, which is using weights designed by the method described in the previous section. Because the array is short relative to the desired wavenumber resolution, the weights are nearly uniform. The striations from the source of interest are much more clearly visible in subfigure (b) than in subfigure (a), because much of the noise has been filtered out. Other array processing (not shown here) confirmed the presence of the interfering sound source seen in subfigure (a).

\subsection{Chapter summary}

Observing intensity striations from the waveguide invariant is useful for many acoustic sensing applications, such as the passive range estimation in $\mathrm{Ch} .3$ and many of the applications discussed in Appendix A. Although this can be done with a single hydrophone, an array can be used to suppress noise coming from directions other than that of the source of interest. In [66], Yang showed that the output of an array processor with uniform array weights contains striations that are similar the striations from a single hydrophone. The present chapter generalized Yang's result for uniform weights to include array processors with arbitrary weights, and showed why is it beneficial to use non-uniform weights. 
(a)

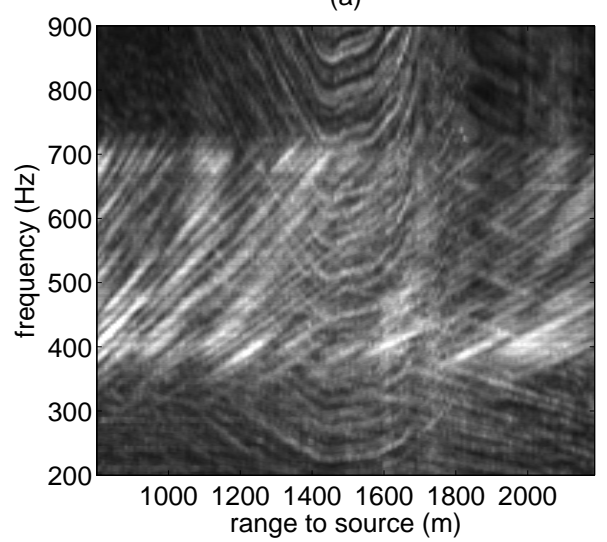

(b)

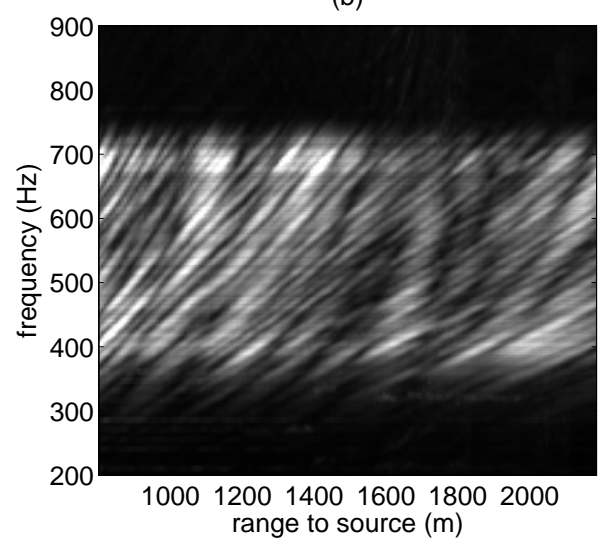

Figure 5.4: Acoustic intensity from experimental data, plotted as a function of frequency and range from the source of interest. If there was no noise in the data, then all that would be seen is striations between 350 to $700 \mathrm{~Hz}$ pointing towards the origin of each subfigure's respective coordinate system. Both subfigures are from the same data set. Subfigure (a) is the acoustic intensity as measured by a single hydrophone from an array towed away from the source. The plot has the expected striations from the source of interest, as well as noise from other interfering sound sources that were present during the experiment. The parabolic shaped striations between 1200 and $1800 \mathrm{~m}$ are from an interfering sound source. Subfigure (b) is the same as (a), but data from all elements of the array were used to filter out noise coming from directions other than that of the source of interest. 
Because the waveguide invariant striations are the result of modes interfering with one another, designing array processing weights for the purpose of observing waveguide invariant striations is different than designing array processing weights for planewave beamforming. It was shown that when an HLA is long enough to distinguish the horizontal wavenumbers of low order modes from the horizontal wavenumbers high order modes, an array processor with uniform weights can have undesired effects. An example was given demonstrating how to design array weights that allow a range of wavenumbers to pass through the array processor, preserving all the desired striation terms while suppressing noise.

It was also shown that even in the absence of noise, it can be beneficial to filter out specific ranges of wavenumbers - ones that correspond to non-SRBR modes - because they tend to cause striations whose slopes are unpredictable and thus corrupt the striations that are useful.

The filtering/beamforming concepts in this chapter were discussed in the context of passive sensing with an array, but they could likely be applied to active arrays in the context of TRM or active sonar, similar to the ideas discussed in [40]. For example, in the context of variable range TRM, one could have the array only emit sound over the range of wavenumbers corresponding SRBR modes which typically have $\beta_{m l} \approx 1$, thus improving the ability to predict how the range of the focal spot will change with frequency. 


\section{Chapter 6}

\section{A relationship between the waveguide invariant and wavenumber integration}

Chapters 3 - 5 analyzed the waveguide invariant and its applications using normal modes. It was pointed out in Sec. 2.3 that the waveguide invariant can also be analyzed using ray theory. This chapter presents original research that attempts to complete the ray/mode/wavenumber-integration 'trifecta.' ${ }^{1}$ Although the research presented in this chapter is mostly of theoretical interest, but may have some practical use in waveguides with attenuating elastic media.

\subsection{Introduction}

Although the waveguide invariant is usually derived and interpreted using normal modes [11], ray theory can also be used to interpret the waveguide invariant $[18,24,14]$. The present analysis relates the waveguide invariant to another common method for calculating the acoustic field in a waveguide: wavenumber integration. It will be shown that in some cases, the waveguide invariant can be "seen"

\footnotetext{
${ }^{1}$ This chapter is based on "A relationship between the waveguide invariant and wavenumber integration" by Kevin L. Cockrell and Henrik Schmidt [The Journal of the Acoustical Society of America Express Letters. July, 2010].
} 
in a plot of the autocorrelation of the wavenumber-integration kernel. This result is somewhat intuitive because the autocorrelation of the wavenumber-integration kernel represents horizontal wavenumber differences, which is what the waveguide invariant is defined in terms of when using a normal mode description of the acoustic field.

The present analysis is for a range independent waveguide with planar geometry so that the wavenumber transform is a Fourier transform (as opposed to a Bessel transform for cylindrical geometry). Because the usual derivation of the waveguide invariant ignores cylindrical spreading, assuming planar geometry is not a limitation.

\subsection{The waveguide invariant and normal modes in a pla- nar waveguide}

A derivation of the waveguide invariant using normal modes was given in Sec. 2.3 (originally [18] and [11, Sec. 6.7.2]), using a point source (cylindrical geometry) in a waveguide. Here we present a similar derivation, but using a planar geometry. For planar geometry, the complex pressure can be written as as shown in [33, Eq. 5.26].

$$
p(x, z, \omega) \propto \sum_{m} \psi_{m}\left(z_{s}\right) \psi_{m}(z) \frac{e^{i k_{x m}(\omega)|x|}}{k_{x m}(\omega)}
$$

where $x$ is the coordinate pointing directly away from the source and $k_{x m}(\omega)$ is the horizontal wavenumber for mode $m$ at a temporal frequency of $\omega$. Analogous to the derivation in Sec. 2.3, define

$$
B_{m} \equiv \psi_{m}\left(z_{s}\right) \psi_{m}(z) \frac{1}{k_{x m}(\omega)}
$$


The scalar acoustic intensity is then the pressure times its complex conjugate:

$$
\begin{aligned}
I(x, \omega) & =p(x, \omega) \cdot \bar{p}(x, \omega) \\
& \propto\left(\sum_{m} B_{m} e^{i k_{x m}(\omega) x}\right) \cdot\left(\sum_{l} B_{l} e^{-i k_{x l}(\omega) x}\right) \\
& =\left(\sum_{q} B_{q}^{2}+2 \sum_{m \neq l} B_{m} B_{l} \cos \left(\Delta k_{m l}(\omega) x\right)\right)
\end{aligned}
$$

where the overline indicates a complex conjugate and $\Delta k_{m l}(\omega)=k_{x m}(\omega)-$ $k_{x l}(\omega)$. Eq. (6.4) shows that the intensity at a fixed $\omega$ is a sum of cosines, each of which has a spatial frequency in the $x$ coordinate that depends on the difference between a pair of modes' horizontal wavenumbers.

It was shown in Sec. 2.3.2 that in an ideal waveguide, $\beta \approx 1$ because $\Delta k_{m l}(\omega) \propto$ $1 / \omega$ for modes far from cutoff. That implies that the $\mathrm{x}$-coordinate spatial frequencies in the intensity depend on $\omega$ like $1 / \omega[26]$.

\subsection{Relating the waveguide invariant to wavenumber in- tegration}

In the previous section it was shown that the $\mathrm{x}$-coordinate spatial frequencies in the acoustic intensity are determined by horizontal wavenumber differences. In this section, we relate the wavenumber integration kernel to the $\mathrm{x}$-coordinate spatial frequencies in the intensity $I(x, \omega)$, which reveals a relationship between the wavenumber integration kernel and the waveguide invariant.

To obtain the complex pressure $p(x, \omega)$ using wavenumber integration, the Helmholtz equation for $p(x, \omega)$ is transformed into the wavenumber domain using the Fourier transform pair [33, Eqs. 2.85 and 2.86]:

$$
\begin{gathered}
p(x)=\int_{-\infty}^{\infty} \Psi\left(k_{x}\right) e^{i k_{x} x} d k_{x} \\
\Psi\left(k_{x}\right)=\frac{1}{2 \pi} \int_{-\infty}^{\infty} p(x) e^{-i k_{x} x} d x
\end{gathered}
$$


The boundary conditions are then written as a function of $k_{x}$, and the resulting equations are solved in the $k_{x}$ wavenumber domain at a single temporal frequency to yield the wavenumber kernel $\Psi\left(k_{x}, \omega\right)$, which represents the magnitude and phase of the spatial frequency components $k_{x}$ of the complex pressure field at a particular temporal frequency $\omega . \Psi\left(k_{x}, \omega\right)$ is then transformed (back) to the $x$ domain using Eq. (6.5), yielding $p(x, \omega)$. The scalar acoustic intensity can then be computed by multiplying the pressure by its complex conjugate: $I(x, \omega)=p(x, \omega) \cdot \bar{p}(x, \omega)$. Note that the wavenumber kernel $\Psi\left(k_{x}, \omega\right)$ for a cylindrical geometry is identical to that for a planar geometry; only the integral transform used to obtain $p(x, \omega)$ from $\Psi(x, \omega)$ is different.

In order to relate the waveguide invariant to the wavenumber integration kernel $\Psi\left(k_{x}, \omega\right)$, we seek to establish a relationship between $\Psi\left(k_{x}, \omega\right)$ and the $\mathrm{x}$ coordinate spatial frequencies in the intensity $I(x, \omega)$. This relationship is provided by the Wiener-Khinchin Theorem [63, 64], which will now be used to show that the magnitude of $\mathrm{x}$-coordinate spatial Fourier Transform of $I(x, \omega)$ can be calculated from the autocorrelation of the wavenumber kernel. Note that although the WienerKhinchin Theorem is well-known for its use in statistical spectrum estimation, our application is purely deterministic.

The autocorrelation of the wavenumber kernel is:

$$
C\left(\Delta k_{x}\right)=\int_{-\infty}^{\infty} \bar{\Psi}\left(k_{x}\right) \Psi\left(k_{x}+\Delta k_{x}\right) d k_{x}
$$

where the overline indicates a complex conjugate. Following the standard derivation of the Wiener-Khinchin Theorem, insert Eq. (6.6) into Eq. (6.7) and perform a 
series of algebraic manipulations [63, 64]:

$$
\begin{aligned}
C\left(\Delta k_{x}\right)= & \int_{-\infty}^{\infty}\left(\frac{1}{2 \pi} \int_{-\infty}^{\infty} \bar{p}\left(x_{1}\right) e^{i k_{x} x_{1}} d x_{1}\right) \\
& \times\left(\frac{1}{2 \pi} \int_{-\infty}^{\infty} p\left(x_{2}\right) e^{-i\left(k_{x}+\Delta k_{x}\right) x_{2}} d x_{2}\right) d k_{x} \\
= & \frac{1}{4 \pi^{2}} \int_{-\infty}^{\infty} \int_{-\infty}^{\infty} \int_{-\infty}^{\infty} \bar{p}\left(x_{1}\right) e^{i k_{x} x_{1}} p\left(x_{2}\right) e^{-i\left(k_{x}+\Delta k_{x}\right) x_{2}} d k_{x} d x_{1} d x_{2} \\
= & \frac{1}{4 \pi^{2}} \int_{-\infty}^{\infty} \int_{-\infty}^{\infty} \int_{-\infty}^{\infty} \bar{p}\left(x_{1}\right) p\left(x_{2}\right) e^{i k_{x}\left(x_{1}-x_{2}\right)} e^{-i \Delta k_{x} x_{2}} d k_{x} d x_{1} d x_{2} \\
= & \frac{1}{4 \pi^{2}} \int_{-\infty}^{\infty} \int_{-\infty}^{\infty} \bar{p}\left(x_{1}\right) \delta\left(x_{1}-x_{2}\right) p\left(x_{2}\right) e^{-i \Delta k_{x} x_{2}} d x_{1} d x_{2} \\
= & \frac{1}{4 \pi^{2}} \int_{-\infty}^{\infty} \bar{p}\left(x_{1}\right) p\left(x_{1}\right) e^{-i \Delta k_{x} x_{1}} d x_{1} \\
= & \frac{1}{4 \pi^{2}} \int_{-\infty}^{\infty} I\left(x_{1}\right) e^{-i \Delta k_{x} x_{1}} d x_{1}
\end{aligned}
$$

Eq. (6.9) shows that the autocorrelation of the wavenumber kernel is proportional to the $\mathrm{x}$-coordinate spatial Fourier transform of the scalar acoustic intensity $I(x)$. If one were to decompose $I(x)$ into all of its spatial frequencies components, the relative magnitude of those spatial frequencies could be determined by the autocorrelation of the wavenumber kernel.

This result can be related to the normal modes description of the acoustic intensity given in Eq. (6.4). The wavenumber kernel $\Psi\left(k_{x}\right)$ has peaks at values of $k_{x}$ corresponding to the modal horizontal wavenumbers. Thus the autocorrelation of $\Psi\left(k_{x}\right)$ will be large at $\Delta k_{x}$ values corresponding to the differences in the modal horizontal wavenumbers - precisely the $\mathrm{x}$-coordinate spatial frequencies of $I(x, \omega)$ shown in Eq. (6.4).

The peaks (local maxima) of the autocorrelation of the wavenumber kernel correspond to the modal horizontal wavenumber differences $\left(\Delta k_{m l}(\omega)\right)$, so the peaks' dependence on frequency will be the same as $\Delta k_{m l}(\omega)$ 's dependence on frequency. Because the waveguide invariant describes $\Delta k_{m l}(\omega)$ 's dependence on frequency, the waveguide invariant also describes how the peaks of the autocorrelation of the wavenumber kernel depend on frequency. The next section analyzes this further. 


\subsection{An ideal example}

The waveguide invariant is well understood for an ideal waveguide because the horizontal wavenumbers can be calculated analytically. For mode pairs in an ideal waveguide where both modes are far from cutoff, $\Delta k_{m l}(\omega)$ is approximately proportional to $1 / \omega$, which corresponds to $\beta \approx 1$ (see Eq. (2.22) or [26]). So one would expect the $\Delta k_{x}$ location of the peaks in $C\left(\Delta k_{x}, \omega\right)$ for an ideal waveguide to depend on $\omega$ like $1 / \omega$. We now show that this is indeed the case.

The wavenumber kernel for an ideal waveguide is [33, Eq. 2.143]

$$
\Psi\left(k_{x}, \omega\right) \propto \begin{cases}\frac{\sin k_{z} z \sin k_{z}\left(D-z_{s}\right)}{k_{z} \sin k_{z} D}, & z<z_{s} \\ \frac{\sin k_{z} z_{s} \sin k_{z}(D-z)}{k_{z} \sin k_{z} D}, & z>z_{s} .\end{cases}
$$

where $k_{z}=\sqrt{\left(\frac{\omega}{c}\right)^{2}-k_{x}^{2}}$ and $D$ is the depth of the waveguide. $\Psi\left(k_{x}, \omega\right)$ has poles when $k_{z} D=m \pi$ for positive integers $m$, or equivalently at $k_{x}$ values corresponding to the modal horizontal wavenumbers. Those poles will then depend on frequency in the same manner as the modal horizontal wavenumbers will, and so the $\Delta k_{x}$ location of the peaks of $C\left(\Delta k_{x}, \omega\right)$ will depend on $\omega$ in the manner predicted by the waveguide invariant $(\propto 1 / \omega)$.

To visualize this dependence, a plot of $\Psi\left(k_{x}, \omega\right)$ is shown in Fig. 6.1(a) along with its autocorrelation in Fig. 6.1(b). The black lines in Fig. 6.1(b) correspond to a few potential striation paths predicted by the waveguide invariant with $\beta=1$ (lines with $\left.\Delta k_{x} \propto 1 / \omega\right)$. It can be seen that there are striations in Fig. 6.1(b) that do not match up well with the black lines. This discrepancy appears to contradict the analysis in the previous section. But if we remember that $\beta$ is only approximately equal to 1 for mode pairs where both modes are far from cutoff, or equivalently when $k_{x}$ is close to $k$, then the discrepancy makes sense because the wavenumber kernel contains horizontal wavenumber components that are close to cutoff and thus are not well described by $\beta=1$. To address this issue, Fig. 6.1(c) shows the wavenumber kernel using only $k_{x}$ values close to $k$ (specifically, $\frac{2}{3} k<k_{x}<k$ ) and Fig. 6.1(d) shows the resulting autocorrelation along with black lines corresponding to $\beta=1$. The striations in Fig. 6.1(d) match very well with the black lines because only horizontal wavenumbers that are far from cutoff are included. 
(a)

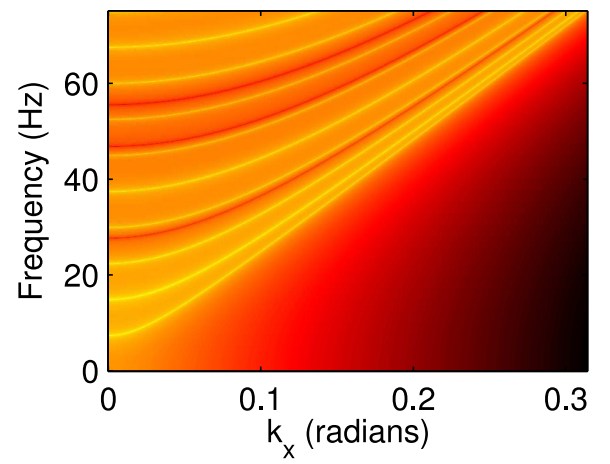

(c)

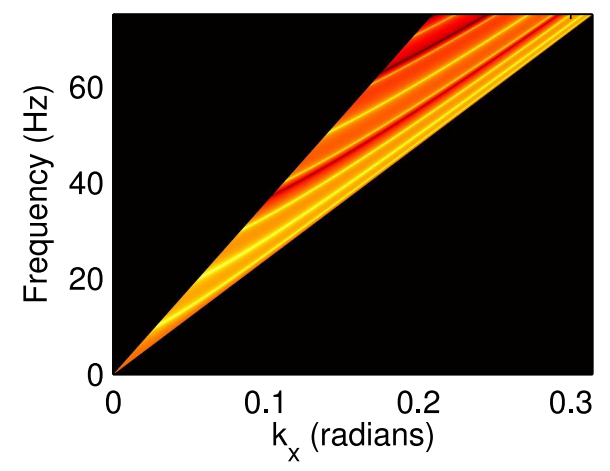

(b)

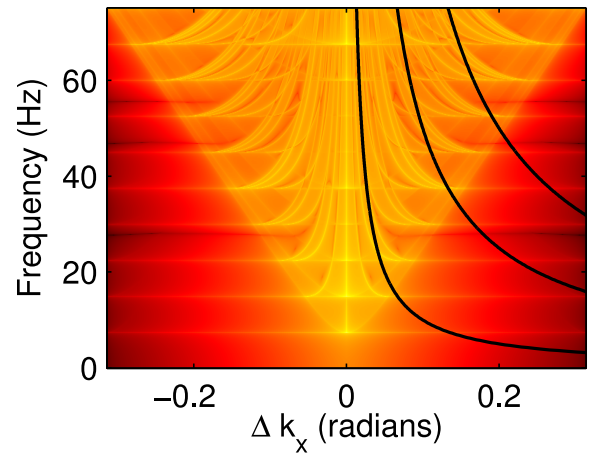

(d)

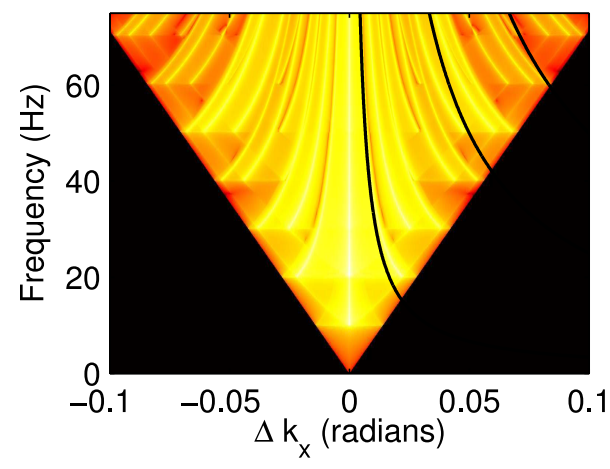

Figure 6.1: All subfigures are for a $100 \mathrm{~m}$ deep ideal waveguide with $z_{s}=16 \mathrm{~m}$ and $z=73 \mathrm{~m}$, and are in units of $\mathrm{dB}$ re: an arbitrary reference. (a) $\left|\Psi\left(k_{x}, \omega\right)\right|$, the wavenumber kernel as a function of horizontal wavenumber and frequency. (b) $\left|C\left(\Delta k_{x}, \omega\right)\right|$, the autocorrelation of wavenumber kernel shown in subfigure (a) as a function of wavenumber difference $\Delta k_{x}$ and frequency $\omega$. The black lines are example striation paths corresponding $\beta=1$ (lines with $\Delta k_{x} \propto 1 / \omega$ ). (c) Same as subfigure (a), but only including $k_{x}$ values far from cutoff $\left(\frac{2}{3} k<k_{x}<k\right)$. (d) $\left|C\left(\Delta k_{x}, \omega\right)\right|$, the autocorrelation of the wavenumber kernel shown in subfigure (c). The black lines are example striation paths corresponding $\beta=1$ (lines with $\Delta k_{x} \propto$ $1 / \omega)$, and match the actual striation paths more closely than those in subfigure (b) because subfigure (d) only includes $k_{x}$ values far from cutoff. 


\subsection{Relevance}

Many applications of the waveguide invariant, such as the one in $\mathrm{Ch} .3$, require an assumption about the value of $\beta$. It is often correct to assume that $\beta \approx 1$ in shallowwater waveguides, but this assumption is not always valid, as discussed earlier in this thesis and in [18]. Consequently, numerical modeling is sometimes used to determine the approximate value of $\beta$ in a given environment [51]. Although this can be done by simulating the acoustic field itself, more insight can often be gained by calculating more fundamental quantities such as the modal horizontal wavenumbers or the ray parameters (horizontal slowness, cycle distance, etc. - see [24, Eqs. 11-15]), which can be related to the value of the waveguide invariant.

However, normal modes and ray theory are not conducive to describing the acoustic field in some environments, such as those involving attenuating elastic media (especially if one is interested in the acoustic field inside of the elastic media). In those cases, understanding how the wavenumber-integration kernel relates to the waveguide invariant may allow one to gain insights that would be difficult to obtain otherwise.

For example, the concepts described in this chapter may be useful for studying the effect that surface ice has on the value of $\beta$. The striations in a plot like Fig. 6.1(d), but generated for an ice-covered waveguide rather than an ideal one, could reveal whether it's reasonable to assume $\beta=1$ in such an environment. More specifically, one could use a plot like Fig. 6.1(d) to determine if hydrophones sitting on surface ice (or embedded in the seafloor) would record the the same range-frequency waveguide invariant striations that a hydrophone in the water column would record.

\subsection{Chapter summary}

The waveguide invariant implies a specific dependence of the acoustic intensity's $\mathrm{x}$-coordinate spatial frequencies on the temporal frequency $\omega$. When the geometry of the problem is planar, the wavenumber integration transform is a Fourier Transform, and so the Wiener-Khinchin Theorem can be used to relate the autocorrelation of the wavenumber kernel to the x-coordinate spatial frequencies in the 
acoustic intensity. A 2-d plot of the autocorrelation of the wavenumber kernels versus "wavenumber lag" $\Delta k_{x}$ and temporal frequency $\omega$ exhibits striation patterns that can be explained by the waveguide invariant.

The relationship between the waveguide invariant and wavenumber integration is not as direct as the waveguide invariant's relationship to normal modes or ray theory. However, the relationship described in the present analysis may be useful in certain situations because wavenumber integration can be used in waveguides that are difficult to model using normal modes or ray theory. 


\section{Chapter 7}

\section{Conclusion}

The waveguide invariant $\beta$ is both embarrassingly simple (why did it take so long to explain the striations in spectrograms!?), and fantastically complicated (hidden behind so many layers of mathematical manipulation, it's a wonder it was ever discovered!).

To explain waveguide invariant range-frequency striations, one must transform the time-domain wave equation into the frequency domain. Then transform that frequency-domain equation into the horizontal wavenumber domain, and then separate it into a depth-dependent equation and a range-dependent equation. The rangedependent equation provides the $e^{i k_{r m} r}$ terms that interfere with one another. The depth-dependent equation, which often cannot be solved analytically, provides the $k_{r m}$ which depend on $\omega$. The difference between two $k_{r m} \mathrm{~s}$, and how that difference depends on $\omega$, determines the waveguide invariant $\beta$. And $\beta$ determines the slopes of the striations in a spectrogram from a hydrophone being towed away from a source.

Small changes in the SSP and in the seafloor properties can cause large changes in the structure of the acoustic field. So it is somewhat surprising that a single scalar value of $\beta$ accurately describes the range-frequency striations that appears in the acoustic field under such a wide range of SSPs and seafloors.

This thesis built upon previous research to understand why $\beta$ is such a robust description of the acoustic field, and to develop fundamental signal processing techniques for the waveguide invariant. 
This chapter summarizes this thesis and discusses its significance. Although this thesis made significant contributions, much is left to be understood; several suggestions for future research are given in this chapter.

\subsection{Thesis summary}

Chapter 3 assumed $\beta=1$, which allowed for passive range estimation. Although similar research had already been performed, Chapter 3 quantified the signal processing techniques and derived some fundamental quantities such as the minimum source bandwidth required to observe range-frequency striations. Chapter 3 also provided an experimental example showing that $\beta \approx 1$ in a realistic waveguide with a non-uniform SSP and a seafloor with unknown acoustic properties.

Chapter 4 analyzed why $\beta \approx 1$ in shallow-water waveguides, and how the SSP and seafloor affect $\beta$. The original paper on the waveguide invariant gave some brief hand-waiving, albeit substantive, arguments for this, and solved for $\beta$ analytically in a few special cases. Other researchers investigated $\beta$ numerically, and analytically using ray theory. In $\mathrm{Ch}$. 4 , a method was derived using normal modes that provided not only a quantitative relationship between the SSP and $\beta$, but also a relationship that could also be understood intuitively through the function $\phi_{1}\left(S_{p}\right)$. This method was used to prove that $\beta>0$ for all surface-reflected bottomreflected (SRBR) modes in a vacuum-bounded waveguide regardless of the SSP. It was also used for a qualitative argument suggesting that $\beta \approx 1$ for SRBR modes in a vacuum-bounded waveguide, regardless of the SSP. (Unfortunately the analysis in chapter 4 did not prove that $\beta \approx 1$ for SRBR modes.)

Chapter 5 drew from the understanding gained in Ch. 4, and showed how an array can be used to not only filter out noise, but to filter out non-SRBR modes leaving only the SRBR modes which are likely have $\beta_{m l} \approx 1$.

Chapter 6 related the waveguide invariant to wavenumber integration, developing another tool to study the waveguide invariant. 


\subsection{Thesis Significance}

Applications: The waveguide invariant has been applied to a broad range of problems in ocean acoustics: passive sonar, active sonar, array processing, time-reversal mirrors, and more. (See Appendix A for a review of the waveguide invariant literature). Many of those applications could benefit from the concepts discussed in this thesis. For example, a transmitting TRM array could emit sound primarily at horizontal wavenumbers that correspond to SRBR modes, ensuring that $\beta \approx 1$ and thus increasing the predictability of the focal spot shift with frequency (details in next section).

Fundamental Understanding: The research in this thesis was applied exclusively to range independent waveguides. However, the waveguide invariant is commonly applied to range dependent and azimuthally dependent waveguides. The method for calculating $\beta$ in Ch. 4 could be applied to understanding how rangefrequency striations behave in such environments. It could also be used for understanding how the range-frequency striations change in time due to oceanographic events like internal waves (details in next section).

Also, the analysis in Ch. 6 relating the waveguide invariant to wavenumber integration may be useful for studying the waveguide invariant under conditions which are not conducive to ray theory or normal modes, such as the acoustic field in a lossy elastic seafloor.

\subsection{Specific suggestions for future research}

This section is a list of suggestions for future research. Some suggestions are natural continuations of the research presented in this thesis, and some are simply "holes" in the current understanding of the waveguide invariant. The difficulty of executing these suggestions ranges from "assign as a homework problem" to "write a multi-year multi-institution research grant proposal."

\section{Range dependent environments}

The waveguide invariant $\beta$ can be defined for mildly range-dependent environments using the adiabatic approximation [18]. D'Spain et al. extended that to range- and 
azimuthally-dependent environments using the " $\mathrm{N}$ by 2D" approximation [21]. The method for calculating $\beta$ presented in Ch. 4 could be applied to (or even simply inserted into) [21, Eq. 14 and/or 18].

The concept of the waveguide invariant may even be able to be extended to strongly range-dependent environments where mode coupling occurs. [53] has an expression for how the modal amplitudes $A_{m}$ change as a function of range due to mode-coupling. One may be able to insert that expression, using the mode shapes as calculated from the WKB approximation, into the derivation of the waveguide invariant shown in Sec. 2.3 to see how much mode couping can occur before the range-frequency striations are destroyed.

\section{Forcing $\beta \approx 1$ without an array (AUV environmental adaptation)}

Chapter 4 showed that surface-reflected bottom-reflected (SRBR) modes tend to have $\beta \approx 1$. Chapter 5 showed that if one wants to observe only SRBR modes, one can use an array to filter out certain wavenumbers. An alternative method for observing the striations from only SRBR modes (which tend to have $\beta \approx 1$ ) is to place the acoustic receiver at the depth of the sound speed maximum. Doing so will minimize the contribution to $I(r, \omega)$ from non-SRBR modes, because nonSRBR modes will have small $\psi(z)$ at that depth. This could be useful for any application where one would like to assume $\beta \approx 1$, like passive range estimation. An Autonomous Underwater Vehicle (AUV) could adaptively determine the SSP maximum, and move to that depth. An AUV with an array could detect a source, adaptively head directly towards it, and move to depth of the SSP maximum.

\section{Tighter bounds on $\beta$ for SRBR modes}

In Sec. 4.4, it was mathematically proven that $\beta\left(S_{p}\right)>0$ for SRBR modes in a vacuum-bounded waveguide, regardless of the SSP. The examples in that chapter also showed that typically $\beta\left(S_{p}\right) \approx 1$ for SRBR modes. A "hand-waiving" argument was used to explain this: SRBR modes behave similarly to modes in an ideal waveguide, which have $\beta \approx 1$ (see last paragraph of Sec. 4.2). By using the equations in Sec. 4.4, it may be possible derive tighter bounds on $\beta$, or to quantitatively explain why $\beta \approx 1$ for SRBR modes regardless of the SSP. 


\section{Waveguide invariant array processing for active transmissions}

The array processing concepts presented in Ch. 5, where one filtered out non-SRBR which typically do not correspond to $\beta \approx 1$, could be applied to many of the waveguide invariant applications discussed in Appendix A. Specifically, transmitting arrays in TRM may be able to apply similar concepts, increasing the ability accurate predict how the range of the focal spot will shift with frequency. The work presented in [49] may also benefit from such filtering, although the mode coupling may subdue the desired effect.

\section{Waveguide invariant array processing in non-uniformly spaced arrays}

The array processing (wavenumber filtering) method in Ch. 5 was based on a standard time-domain filter design technique ( $\mathrm{firls}$ in Matlab). Because time domain filter design techniques usually assume the samples are spaced uniformly in time, they cannot be applied to arrays where the elements are non-uniformly spaced. If one wanted to do wavenumber filtering similar to that in $\mathrm{Ch}$. 5 , but for a nonuniformly spaced array, one would have to use (or develop) filter design techniques that can handle non-uniformly spaced arrays. The methods in [61, Ch. 3] may be useful for this.

\section{$1 / r$ spreading}

Quantify the effect of the $1 / r$ spreading that is ignored when deriving the waveguide invariant, in terms of how it affects the "observed" value of $\beta$ in the striation slopes in $I(r, \omega)$. Note that one can factor out the $1 / \sqrt{r}$ in the $A_{m}$ and $A_{l}$ terms in Eq. 2.10 .

\section{Including attenuation when deriving $\beta$}

Re-derive the waveguide invariant but allow for the horizontal wavenumbers to be complex so that they can take into account bottom loss. The result will likely be like Eq. 2.10, but with an exponential decay tacked on to each cosine term. The footnote in Sec. 2.3.1 may help with this. 


\section{Group speeds and phase speeds}

[10, Eq 4.5.15] gives an integral expression for the group speed times the phase speed, $u_{m} \cdot v_{m}$, for a $c(z)$ that is constant above some depth but can vary with $z$ below that depth. That expression may be able to be used to calculate $\beta$, without even using the WKB approximation. Or it may provide some general bounds or insights on the waveguide invariant (in that specific environment).

\section{Striation slopes change with range and frequency}

As discussed in Sec. 2.2.3 and Sec. 2.3.3, the waveguide invariant $\beta_{m l}$ is typically defined using adjacent modes (the Taylor series expansion of group and phase slowness plotter versus mode number). The justification for this is based on a property of the modal sum discussed in Sec. 2.2.3. The group (or groups) of modes that dominate the modal sum is determined by the range and frequency of the receiver. Thus, the range-frequency striation slopes (locally in the $(r, f)$ plane) will be different at different ranges and frequencies, as is observed in Fig. 2.6(c). This phenomena is not observed as strongly in shallow-water waveguides, presumably because shallow-water waveguides have many fewer modes and less of those modes are non-SRBR modes.

It would be worthwhile to quantitatively investigate when the argument in Sec. 2.2.3 applies, and how it could be used to predict which group of modes (and thus which value of $\beta$ ) is relevant at a given range frequency. Perhaps there are certain ranges and frequencies where one can assume $\beta \approx 1$ in a deep-water waveguides, as suggested by some of the striations around $60 \mathrm{~km}$ in Fig. 2.6(c) (which happens to be the convergence zone distance...). This investigation could be done using the argument in Sec. 2.2.3 - looking at $k_{r}$ as a function of $m$ and $\omega$. In addition, ray theory may also provide good insights into this, especially as it is portrayed in [20, Fig. 3.8] (which is the original reference for Sec. 2.2.3).

Another approach for studying this would be to investigate the relationship between the $\left(A_{m} A_{l} \cos \left(\Delta k_{m l} r\right)\right)$ terms in Eq. 2.10, and the argument in Sec. 2.2.3. Figure 2.2 shows the contribution to the complex pressure from each mode, but each $\left(A_{m} A_{l} \cos \left(\Delta k_{m l} r\right)\right)$ term represents the contribution to the magnitude of the pressure from 2 modes. 


\section{Upper limits on range and frequency for observing striations}

Waveguide invariant striations occur when the environment is not strongly rangeand/or azimuthally-dependent (i.e., no mode coupling), and the source is broadband. As the frequency and range increase, scattering from inhomogeneities in the environment (boundary roughness, SSP variations) tends to smear out or completely destroy the waveguide invariant striations. This effect can be seen in Fig. 2.5, where the striation widths get wider as the range increases (contrary to what Eqs. (3.6) and (3.5) predict for a perfectly homogeneous waveguide). Rouseff and Kuz'kin have done some research on this topic [52, 42, 41], but there is certainly more research that could be done with relative ease given the state of understanding of acoustic scattering in the ocean waveguide.

The upper limit in range and frequency at which the striations would appear will depend on the inhomogeneities of a particular environment. Such upper limits could obviously be measured experimentally by towing a hydrophone away from a very broadband source. In addition, the literature in Appendix A provides much experimental data.

On the theoretical side, there is a large body of literature studying scattering in the ocean; surely it could be applied to determining how far away and how high frequency waveguide invariant striations could be observed. To first order, surface and seafloor roughness act simply as a loss-mechanism [33, Sec. 1.7]. More sophisticated models would likely need to be used. [60, Ch. 6] may provide a good starting point, as would [36, Chs. 4 and 6].

Another approach might be to use the expressions used to generate [16, Fig. 5(a)], which could be analyzed as a function range and frequency. $[2,17]$ may also provide useful insights. 


\section{Appendices}





\section{Appendix A}

\section{Review of waveguide invariant literature}

This appendix is a list of references about the waveguide invariant, with each references loosely categorized based on its application. Nearly all articles in The Journal of the Acoustical Society of America and IEEE Oceanic Engineering relating to the waveguide invariant are included here; some of the Russian literature is also included. ${ }^{1}$ [47] gives a brief summary of the Russian literature on the waveguide invariant and similar phenomena, including several of references which are not reviewed in this appendix.

\section{A.1 General review}

\section{Fundamental research on the waveguide invariant}

The first paper on the waveguide invariant is [18] by Chuprov. This paper discusses the waveguide invariant from the perspective of normal modes and ray theory. It focuses primarily on range-frequency striations, but also discusses some changes with receiver depth. It focuses mostly on deep-water propagation but discusses shallow-water waveguides as well. It analytically calculates the waveguide invari-

\footnotetext{
${ }^{1}$ The journal Soviet Physical Acoustics turned into Acoustical Physics sometime in the 1990s. Articles from Acoustical Physics are available online from Springer; many articles in Soviet Physical Acoustics have been translated into English and were able to be tracked down MIT's superb librarians.
} 
ant for ideal waveguides and surface-trapped modes in an $n^{2}$-linear waveguide. It presents numerical and experimental data, and shows that in most cases the Doppler effect due to source/receiver motion has little effect on range-frequency striations. It also briefly mentions how to account for mild range dependence using the adiabatic approximation (although other references give a more detailed derivation). In general, this paper is dense and fairly difficult to follow unless the reader has a very deep understanding of ocean acoustics. It's a 'must read', but probably only after reading other simpler derivations of the waveguide invariant.

In [11, Sec. 6.7.2], Brekhovskikh and Lysanov give a derivation and discussion of the range-frequency waveguide invariant in somewhat simpler terms than Chuprov's original paper does. ${ }^{2}$

[21] by D'Spain et al. is a highly cited paper which presents more thorough derivations of many of the results in Chuprov's original paper. This paper extends Chuprov's range-dependent expression for $\beta$ to environments that change with azimuth as well, using the " $\mathrm{N}$ by $2 \mathrm{D}$ " approximation. Striations in experimental data with a frequencies of $70-170 \mathrm{~Hz}$ are compared with predicted striation slopes. Also derived is an expression for the striation trajectories in the spectrogram of a fixed hydrophone, when the a source's position is a function of time. This paper provides an approximate expression for $\beta$ in an waveguide with a constant sound speed and vacuum bottom, but with range-dependent bathymetry. Also discussed are "generalized" waveguide invariants, which explain striations which results from changes in waveguide depth.

The 2nd edition of the textbook "Computational Ocean Acoustics", which is still in preparation, will contain a few sections that discuss the waveguide invariant. [34, Sec. 2.4.6] gives a very nice introduction to the waveguide invariant. There is a section in [34, Ch. 3] that discusses how the waveguide invariant can be calculated from acoustic ray parameters, which is based on the work in [22] and [24]. Expressions are derived for calculating the waveguide invariant without needing to run full ray-tracing models, similar to the result in Sec. 4 of this thesis which does not require solving for the horizontal wavenumbers. A section in [34, Ch. 5] also discusses the waveguide invariant, and gives some numerical examples.

In [26], Grachev shows that if a single scalar value of $\beta$ is an accurate descrip-

\footnotetext{
${ }^{2}$ This author recalls noticing an typographical/mathematical error in their derivation, however.
} 
tion of the horizontal wavenumbers, then those horizontal wavenumbers must have a power-law dependence on frequency. Grachev also discusses an analogous result or a generalized waveguide invariant that deals with changes in waveguide depth. Also discussed are $\beta$ for surface-trapped modes modes in $n^{2}$-linear waveguides, and $\beta$ for waterborne modes in asymmetric and symmetric $n^{2}$-linear waveguides.

In [42], Kuz'kin et al. study range-frequency striations in a range-dependent environment using experimental data. Perturbation theory is used to determine how changes in the SSP will affect changes in the observed striation pattern, and how one might be able to use that for acoustic inversions.

In [41], Kuz'kin uses a statistical correlation framework to study range-frequency striations (or frequency shifts) due to range-dependent SSP perturbations.

In [15], Burnekov discusses the boundaries (range) of validity of the adiabatic range-dependent expression for $\beta$. He analyzes $\beta$ in the case of a waveguide with a constant bottom slope, and he shows that $\beta$ as a function of range can change quickly with range even if the environment changes slowly with range because modes can go from being SRBR to non-SRBR as the bathymetry changes.

In [44], Lobanov and Petukhov discuss that in range-dependent environments, $\beta$ can have a "singularity" $\left(\beta\left(r_{1}\right)=0\right)$ which implies the range-frequency striations will be completely parallel to the frequency axis in the range-frequency plane. They point out that in the time domain, $\beta\left(r_{1}\right)=0$ means that pulse signals are not subject to dispersion broadening with time at the range $r_{1}$. [44] investigates the range-frequency striations when only a few modes are propagating, and notes that even if many modes are propagating, the higher order modes often attenuate away with range leaving only a few propagating modes. The effects of seismic (elastic) waves on the range-frequency-striations are also briefly discussed. Finally, there is a brief mention of how $\beta$ can change with range and frequency.

Note that much of the Russian literature, such as [44], makes statements that only apply to waterborne modes without explicitly stating that the statements only apply to waterborne modes. For example: "under the WKB approximation, a group speed has a functional dependence on the phase speed [independent of frequency]." That statements is only true for waterborne modes, and is not true for bottom interacting modes (Ch. 4 illustrates why).

In [52], Rouseff uses numerical simulations to study the effect of shallow-water 
internal waves on $\beta$. He discusses that the value of $\beta$ depends on the type of mode, and that the "measured" value of $\beta$ (i.e., the striations slope seen in $I(r, \omega)$ ) depends on receiver depth. He numerically studies the effect of bottom attenuation and range on the range-frequency slopes.

In [4], Baggeroer discusses methods for plotting and understanding the waveguide invariant in several canonical waveguides, using normal mode simulations. Baggeroer also shows how the 2D-DFT can be used to extract the value or distribution of $\beta$ from $I(r, \omega)$.

In [51], Rouseff and Spindel discuss how to use a 2D-DFT to measure the distribution of $\beta$ from $I(r, \omega)$, and show that the striations slopes sometimes depends on the source and receiver depths.

In [14], Brown et al. shows that when the modal horizontal wavenumbers are calculated from from the WKB approximation and the limit of high frequency is taken, $\beta$ is equivalent to the "ray stability parameter" $\alpha$. Note that there is some overlap between the work in [14] and the work Ch. 4. [14] discusses rangedependent effects, including mode-coupling. [6] extends the work of [14] and discusses how $\beta$ related to "acoustic beam dynamics."

In [1], An et al. discuss how a 2D-DFT can be used to calculate the slope of the waveguide invariant striations. They show a simulated plot of $\beta$ versus frequency and modal phase speed in a Pekeris waveguide. They also show how the value of $\beta$ observed in $I(r, \omega)$ depends on the source and receiver depths, and they show some experimental data from the South China Sea with frequencies around $370 \mathrm{~Hz}$ and ranges around $13 \mathrm{~km}$.

In [13], Brooks et al. discusses a few methods of extracting the striation slopes from $I(r, \omega)$.

[47], which is a summary of the Russian literature on the waveguide invariant, is the first chapter of a book which contains several articles on the waveguide invariant, some of which are not described in this appendix.

\section{Active sonar}

In [49], Quijano et al. discuss how the waveguide invariant concept can be applied to bi-static active sonar. An expression is given for the pressure as a function of source/receiver/target geometry, including the mode coupling which depends on the 
model of the target. That expression illustrates how $\beta$ from passive measurements can be related to active sonar. Some experimental data shows a measured $\beta_{\text {active }}$ of 1.4, which is then compared to the (passively) measured values of $\beta$ from other papers.

In [27], Chensong et al. applies the concept of the active sonar waveguide invariant from [49] to an extended Kalman filter tracking algorithm. The waveguide invariant is used to restrict the possible state transitions of the state vector in the Kalman filter, leading to better tracking performance.

In [25], Goldhahn et al. uses the waveguide invariant to describe reverberation in active sonar returns, allowing for an improved ability for target detection.

\section{Passive sonar and non-adaptive array processing}

In [56], Tao et al. demonstrate a method for estimating the speed and closest-pointof-approach of a moving source. Tao shows that if $\beta=1$ and the source maintains a constant speed and bearing, parabolic striations will appears in a spectrogram from single fixed hydrophone. The properties of the striations are extracted using a Hough transform, and a range and speed estimate are produced. The method is tested on experimental data from a pontoon boat in a $9 \mathrm{~m}$ deep lake, with frequencies of about $3-5 \mathrm{kHz}$ and ranges of a few hundred meters.

In [23], D'Spain et al. discuss striations in a plot the beamformed acoustic intensity from a vertical array. Striations in range, frequency and vertical wavenumber are discussed assuming the vertical array is an ideal wavenumber-filter (as opposed to [66] and Ch. 3, which explicitly take into account the array's non-ideal response). The results are discussed in the context of 4 different SSPs which can be treated analytically. Methods for depth and range estimation are discussed and demonstrated using experimental data from SWellEx-3 (frequencies of 300-700 Hz and ranges of 1.5 to $6 \mathrm{~km}$.)

In [66], Yang shows that the waveguide invariant range-frequency striations appear in the output of a horizontal line array beamformer, with some array gain. He discusses some applications of this and demonstrates them with simulated data.

In [62], Turgut et al. estimate the ratio of a broadband acoustic source's speed to its closest-point-of-approach. Striation trajectories are derived for sources with constants speeds and bearings, similar to those in [56]. The striations observed in 
the beamformer's output from experimental data (frequencies of $50-150 \mathrm{~Hz}$ ) are analyzed using Hough transforms. The waveguide invariant formulation from [21] is used to take into account a $\beta$ that varies with range and azimuth. [62] also investigates the possibility of jointly estimating the value of $\beta$ along with the other parameters.

Chapters 3 and 5 of this thesis, and their associated papers in JASA would be in this section.

In [59], Thode et al. localize an acoustic source by utilizing the waveguide invariant in a matched field processing (MFP) scheme. Thode shows that the sidelobes of the MFP, when plotted versus frequency for a broadband source, follow a trajectory described by $\beta$ which can be used to accurately estimate the range to the acoustic source assuming $\beta=1$. Thode demonstrates this experimentally for shallow water with a Blue whale vocalization. Thode also argues through analysis and simulation that even if $\beta$ is a negative number, as it would be in environments like the Arctic, one can use the sidelobe behavior to estimate the source range. This result is interesting because most other applications of the waveguide invariant assume $\beta=1$.

In [58], Thode presents a method for "estimating the range of an unknown broadband acoustic source in a [range-independent] waveguide, using a vertical array and a signal sample from another broadband source a known location relative to the array." Experimental data is used with frequencies from $75-150 \mathrm{~Hz}$ and ranges from about $3-5 \mathrm{~km}$. Thode discusses the use of a Radon transform to find the slopes of striations.

\section{Time-reversal mirrors and adaptive array processing}

In [57], Tao and Krolik used the waveguide invariant to improve covariance matrix estimation for adaptive beamforming, in order to reduce coherent multipath interference. The basic idea is that the covariance matrix at one frequency can provide useful information about the covariance matrix at another frequency, with the value of $\beta$ describing how the wavenumber differences change with frequency.

In [37], Kim et al. use the waveguide invariant for null-broadening - an adaptive beamforming technique - in the context of matched field processing in a waveguide. A relationship to adaptive time-reversal mirrors is also discussed. 
In [54], Song et al. use the waveguide invariant to create a time-reversal mirror with variable range focusing. The idea is that by shifting the frequency of the received signal before re-transmitting it, the range at which the sound will focus will shift proportional to the value of $\beta$. The range is about $6 \mathrm{~km}$ and the frequency is about $450 \mathrm{~Hz}$.

In [32], Hodgkiss et al. experimentally verify the ideas presented in [54], and perform the TRM focusing at ranges of up to $30 \mathrm{~km}$, at frequencies around $450 \mathrm{~Hz}$. By comparing the change in frequency with the change in range of the focal point, they measure $\beta=1.4$.

In [38], Kim et al. use the waveguide invariant to predict the cross-spectral density matrix (CSDM) at one range based on the measured CSDM at a slightly different range from a 'probe' source (assuming the depth remains fixed). This predicted CSDM is used to place a null with an adaptive TRM.

In [39] by Kim et al. changes in focal depth due to changes in frequency and waveguide depth are related to the waveguide invariant.

\section{Geoacoustic Inversion}

In [29], Heaney uses the waveguide invariant striations' slopes and spacing, along with the incoherent transmission loss, for "rapid geoacoustic inversion". In [28], Heaney extends that work to range-dependent environments, and in [30] Heaney et al. use a seismic survey source.

\section{The array invariant and the waveguide invariant}

[43] describes an invariant property of acoustics fields in a waveguide which is dubbed "The array invariant", which is used for passive range estimation for an impulsive acoustic source. The array invariant describes a property of the acoustic signal as a function of time and range along an array. The array invariant differs from the waveguide invariant in that the array invariant describes the acoustic pressure itself, as opposed to the acoustic intensity. The array invariant is only invariant for modes where $k_{z}$ has (almost) no dependence on frequency, as discussed in [43]. These modes are usually the same modes for which $\beta \approx 1$. 


\section{A.2 Rays, modes, and the waveguide invariant}

In [18], Chuprov states that the waveguide invariant can be described using normal modes or ray theory. Subsequent research can be found in [22] and [24], the results of which are summarized in [34, Ch. 3]. Also see [14] and [6].

[20, Fig. 3.8] provides a very interesting way of understanding the relationship between rays and the modal sum, and that understanding may be very useful for understanding how the observed striations in $I(r, \omega)$ depend on range and frequency - a topic that is not well studied as pointed out in Sec. 2.3.3. For example, $\beta$ is defined in terms of a group of adjacent modes. The striations in $I(r, \omega)$ are determined by whichever group of modes dominates the modal sum at particular $r$ and $\omega$, as discussed in Sec. 2.2.3. Because a ray represents the interference between adjacent modes, ray tracing may be a good tool for determining how the striations in $I(r, \omega)$ change with $r$ and $\omega$. This author is not aware of any research exploring this idea in detail.

The WKB approximation can be a "bridge" between normal modes and ray theory, as discussed [33, Ch. 3] and even more so in [34, Ch. 3]. [60, Sec. 5.3] and other parts of [60] also discuss the relationship between rays and modes, as does [11, Sec. 6.7.1]. 


\section{Appendix B}

\section{References for the WKB approximation in ocean acoustics}

The WKB approximation is used in parts of this thesis to describe the horizontal wavenumbers of modes in ocean acoustic waveguides. It is a general mathematical technique for approximating the solution to certain types of differential equations, and is discussed in textbooks on applied math, quantum mechanics, electromagnetism, and acoustics.

This appendix is a far-from-exhaustive list of references that discuss the WKB approximation specifically as it applies to ocean acoustics.

- $[9$, Ch. 8] and [8, Ch. 23 ] discuss the validity of the WKB approximation as applied to acoustic propagation in stratified media, but not specifically as it applies to modes waveguides.

- [8, Sec. 48.5] discusses WKB approximation for surface-trapped normal modes in an $n^{2}$-linear waveguide.

- [8, Ch. 49.6] discusses WKB approximation for surface-trapped modes in an Epstein waveguide.

- [60, Sec. 2.9 and 5.3] discuss the WKB approximation for ocean acoustic waveguides, as it relates to modes and ray theory. 
- [33, Sec. 2.5 and Ch. 3] discuss the WKB approximation and how it relates to ray theory. [34, Ch. 3] has even more material on how the WKB approximation relates to ray theory.

- $[33$, Sec. 5.6] uses the WKB approximation to describe the mode shapes in deep-water waveguides.

- [11, Sec. 6.7] briefly discusses the WKB approximation, and also has a nice bulleted list of mode types. However, it does not discuss bottom interacting modes and thus does not discuss how to use the vertical phase integral to obtain the modal horizontal wavenumbers. It does, however, discuss a good technique for normalizing the mode shapes obtained from the WKB approximation.

- [5] discusses how to use the WKB approximation to use calculate the modal horizontal wavenumbers for modes in ocean acoustic waveguides.

This WKB approximation as presented in Sec. 4.2 of this thesis is based on [5]. But [60, Sec. 2.9] also provides most of the relevant information. 


\section{Appendix C}

\section{List of symbols and acronyms}

modal the adjective for the noun "normal modes" (e.g., modal horizontal wavenumbers).

JASA The Journal of the Acoustical Society of America

MFP matched field processing

SSP sound speed profile

TRM time-reversal mirror

2D-DFT two-dimensional discrete Fourier transform

$c$ speed of sound

$d$ depth of ocean waveguide

$I$ scalar acoustic intensity

$l$ mode number (same as $m$, but used when there is a double summation)

$m$ mode number

$n$ mode number, but in terms of the WKB approximation. See Sec. 4.2. In $n^{2}$ linear waveguide, $n$ stands for the index of refraction.

$r$ range from the acoustic point source in a waveguide 
$x$ range from the acoustic line source in a waveguide. See Ch. 6 .

$S_{p}$ phase slowness (See Sec. 2.3.3)

$S_{g}$ group slowness (See Sec. 2.3.3)

$z$ depth below the sea-surface

$k_{r \text {,image }}$ the image wavenumber of $I_{\text {win }}(r, f)$ in the $r$ direction. See Sec. 3.3.2

$k_{f \text {,image }}$ the image wavenumber of $I_{\text {win }}(r, f)$ in the $f$ direction. See Sec. 3.3.2

$\beta$ the waveguide invariant when approximated as being independent of the mode numbers and frequency.

$\beta_{m l}$ the waveguide invariant for a pair of modes with mode numbers $m$ and $l$.

The array processing in Ch. 5 uses the following symbols (bold faced lowercase letters denote vector quantities):

- $c_{0}$ is the speed of sound at the HLA depth

- $k_{0}=\frac{\omega}{c_{0}}$ is the wavenumber at the HLA depth

- $J$ is the number of elements in the HLA, with each element numbered as $j=1, j=2, \ldots, j=J$

- $\theta_{L}$ is the look direction as measured from broadside of the HLA

- $k_{L}=k_{0} \sin \theta_{L}$ is the horizontal wavenumber corresponding to the look direction

- $\mathbf{v}\left(k_{L}\right)=\left[v_{1}\left(k_{L}\right), v_{2}\left(k_{L}\right), \cdots, v_{J}\left(k_{L}\right)\right]^{T}$ is the steering vector corresponding to the look direction. $\mathbf{v}$ is a column vector

- $\mathbf{w}=\left[w_{1}, w_{2}, \cdots, w_{J}\right]^{T}$ the array weights column vector (e.g., Hamming, Hann, uniform)

- $\theta_{S}$ is the bearing of the acoustic source as measured from broadside of the $\mathrm{HLA}$, in the plane parallel to the ocean surface 
- $\Upsilon$ is the wavenumber response function of the array processor, which depends on the array geometry and the array weights $\mathbf{w}$, and is described in more detail later. 


\section{Bibliography}

[1] Liang An, Zhi qiang Wang, and Ji ren Lu. Calculating the waveguide invariant by passive sonar lofargram image. In Mechatronics and Machine Vision in Practice, 2007. M2VIP 2007. 14th International Conference on, pages 13 17, 4-6 2007.

[2] Mark Andrews, Tianrun Chen, and Purnima Ratilal. Empirical dependence of acoustic transmission scintillation statistics on bandwidth, frequency, and range in new jersey continental shelf. The Journal of the Acoustical Society of America, 125(1):111-124, 2009.

[3] A. B. Baggeroer, W. A. Kuperman, and Henrik Schmidt. Matched field processing: Source localization in correlated noise as an optimum parameter estimation problem. The Journal of the Acoustical Society of America, 83(2):571-587, 1988.

[4] Arthur B. Baggeroer. Estimation of the distribution of the interference inveriant with seismic streamers. In W. A. Kuperman and G. L. D'Spain, editors, Ocean Acoustic Interference Phenomena and Signal Processing, volume 621 of AIP Conf. Proc., pages 151-170, June 2002.

[5] Charles L. Bartberger. The computation of complex normal mode eigenvalues in underwater acoustic propagation. In D. Lee, R.L. Sternberg, and M.H. Schultz, editors, Computational Acoustics: Algorithms and applications. Elsevier Science Publishers, North-Holland, 1988. 
[6] Francisco J. Beron-Vera and Michael G. Brown. Underwater acoustic beam dynamics. The Journal of the Acoustical Society of America, 126(1):80-91, 2009.

[7] John M. Brayer. Introduction to fourier transforms for image processing http://www.cs.unm.edu/ brayer/vision/fourier.html. 2009.

[8] L. M. Brekhovskikh. Waves in Layered Media. Academic Press, New York, 2nd edition, 1980.

[9] L. M. Brekhovskikh and O.A. Godin. Acoustics of Layered Media I. Springer, New York, 2nd edition, 1998.

[10] L. M. Brekhovskikh and O.A. Godin. Acoustics of Layered Media II. Springer, New York, 2nd edition, 1999.

[11] L.M. Brekhovskikh and Yu.P. Lysanov. Fundamentals of Ocean Acoustics. AIP Press/Springer, New York, 3rd edition, 2003.

[12] William L. Briggs. The DFT : an owner's manual for the discrete Fourier transform. Society for Industrial and Applied Mathematics, 1995.

[13] Laura Brooks, M.R.F. Kidner, Anthony C. Zander, Colin H. Hansen, and Z. Yong Zhang. Techniques for extraction of the waveguide invariant from interference patterns in spectrograms. 2006.

[14] Michael G. Brown, Francisco J. Beron-Vera, Irina Rypina, and Ilya A. Udovydchenkov. Rays, modes, wavefield structure, and wavefield stability. The Journal of the Acoustical Society of America, 117(3):1607-1610, 2005.

[15] S. V. Burenkov. Distinctive features of the interference structure of a sound field in a two-dimensionally inhomogeneous waveguide. Soviet Physical Acoustics, 35(5):465-467, 1989.

[16] Tianrun Chen, Purnima Ratilal, and Nicholas C. Makris. Mean and variance of the forward field propagated through three-dimensional random internal waves in a continental-shelf waveguide. The Journal of the Acoustical Society of America, 118(6):3560-3574, 2005. 
[17] Tianrun Chen, Purnima Ratilal, and Nicholas C. Makris. Temporal coherence after multiple forward scattering through random three-dimensional inhomogeneities in an ocean waveguide. The Journal of the Acoustical Society of America, 124(5):2812-2822, 2008.

[18] S. D. Chuprov. Interference structure of a sound field in a layered ocean. In L. M. Brekhovskikh and I. B. Andreeva, editors, Ocean Acoustics. Current State, Nauka, Moscow, 1982.

[19] Kevin L. Cockrell and Henrik Schmidt. Robust passive range estimation using the waveguide invariant. The Journal of the Acoustical Society of America, 127(5):2780-2789, 2010.

[20] F. R. DiNapoli and R. L. Deavenport. Numerical models of underwater acoustic propagation. In J. A. DeSanto, editor, Ocean Acoustics. Springer-Verlag, New York, 1979.

[21] G. L. D'Spain and W. A. Kuperman. Application of waveguide invariants to analysis of spectrograms from shallow water environments that vary in range and azimuth. The Journal of the Acoustical Society of America, 106(5):24542468, 1999.

[22] G. L. D’Spain, G. L. Rovner, P. Gerstoft, W. A. Kuperman, and W. S. Hodgkiss. Determination of the waveguide invariant in general environments. US Navy J. Underwater Acoust., 51:123-142, January 2002.

[23] G.L. D’Spain, W.A. Kuperman, and J.J. Murray. Matchless field processing in shallow water. In OCEANS 2000 MTS/IEEE Conference and Exhibition, volume 1, pages $653-660$ vol.1, 2000.

[24] P. Gerstoft, G. L. D’Spain, W. A. Kuperman, and W. S. Hodgkiss. Calculating the waveguide invariant (beta) by ray-theoretic approaches. MPL Tech. Memo. TM-468, Marine Physical Laboratory, University of California, San Diego, December 2001.

[25] Ryan Goldhahn, Granger Hickman, and Jeffrey Krolik. Waveguide invariant broadband target detection and reverberation estimation. The Journal of the Acoustical Society of America, 124(5):2841-2851, 2008. 
[26] G. A. Grachev. Theory of acoustic field invariants in layered waveguides. Acoustical Physics, 39(1):33-35, 1993.

[27] Chensong He, J.E. Quijano, and L.M. Zurk. Enhanced kalman filter algorithm using the invariance principle. Oceanic Engineering, IEEE Journal of, 34(4):575 -585, oct. 2009.

[28] K.D. Heaney. Rapid geoacoustic characterization: applied to range-dependent environments. Oceanic Engineering, IEEE Journal of, 29(1):43 - 50, jan. 2004.

[29] K.D. Heaney. Rapid geoacoustic characterization using a surface ship of opportunity. Oceanic Engineering, IEEE Journal of, 29(1):88 - 99, jan. 2004.

[30] K.D. Heaney, D.D. Sternlicht, A.M. Teranishi, B. Castile, and M. Hamilton. Active rapid geoacoustic characterization using a seismic survey source. Oceanic Engineering, IEEE Journal of, 29(1):100 - 109, jan. 2004.

[31] F.B. Hildebrand. Advanced Calculus for Applications. Prentice Hall, Upper Saddle River, New Jersey, 2nd edition, 1976.

[32] W. S. Hodgkiss, H. C. Song, W. A. Kuperman, T. Akal, C. Ferla, and D. R. Jackson. A long-range and variable focus phase-conjugation experiment in shallow water. The Journal of the Acoustical Society of America, 105(3):1597-1604, 1999.

[33] F.B. Jensen, W.A. Kuperman, Michael B. Porter, and H. Schmidt. Computational Ocean Acoustics. AIP Press/Springer, New York, 2000.

[34] F.B. Jensen, W.A. Kuperman, Michael B. Porter, and H. Schmidt. Computational Ocean Acoustics (NOTE: This edition is expected to be published in 2011, but is still in preparation. As such, the contents may change.). AIP Press/Springer, New York, 2nd edition, 2011.

[35] A. C. Kak and Malcolm Slaney. Principles of Computerized Tomographic Imaging. IEEE Press, 1988. 
[36] Boris G. Katsnelson and Valery G. Petnikov. Shallow Water Acoustics. Springer Praxis Books / Geophysical Sciences, 2002.

[37] J. S. Kim, W. S. Hodgkiss, W. A. Kuperman, and H. C. Song. Nullbroadening in a waveguide. The Journal of the Acoustical Society of America, 112(1):189-197, 2002.

[38] J. S. Kim, H. C. Song, and W. A. Kuperman. Adaptive time-reversal mirror. The Journal of the Acoustical Society of America, 109(5):1817-1825, 2001.

[39] Seongil Kim, W. A. Kuperman, W. S. Hodgkiss, H. C. Song, G. F. Edelmann, and T. Akal. Robust time reversal focusing in the ocean. The Journal of the Acoustical Society of America, 114(1):145-157, 2003.

[40] W.A. Kuperman, S. Kim, G. Edelmann, W.S. Hodgkiss, H.C. Song, and T. Akal. Group and phase speed analysis for predicting and mitigating the effects of fluctuations. In N.G. Pace and F.B. Jenson, editors, Impact of Littoral Environmental Variability on Acoustic Predictions and Sonar Performance, pages 279-286, 2002. Available at http://wwwmpl.ucsd.edu/people/hcsong/publications.html.

[41] V. M. Kuz'kin. Spectral intensity oscillations of a sound field in a randomly inhomogeneous ocean. Acoustical Physics, 46(3):310-316, 2000.

[42] V. M. Kuz'kin, A. V. Ogurstov, and V. G. Petnikov. The effect of hydrodynamic variability on frequency shifts of the interference pattern of a sound field in a shallow sea. Acoustical Physics, 44(1):77-82, 1998.

[43] Sunwoong Lee and Nicholas C. Makris. The array invariant. The Journal of the Acoustical Society of America, 119(1):336-351, 2006.

[44] V. N. Lobanov and Yu. V. Petukhov. Space-frequency distribution of the intensity of wideband sound in a shallow sea. Acoustical Physics, 39(6):574-581, 1993.

[45] Walter Munk, Peter Worcester, and Carl Wunsch. Ocean Acoustic Tomography. Cambridge Univeristy Pretty, New York, 1995. 
[46] Alan V. Oppenheim, Ronald W. Schafer, and John R. Buck. Discrete-Time Signal Processing. Prentice Hall, 2nd edition, 1999.

[47] Yury V. Petukhov. Fundamental directions of investigations of interference phenomena observed in the propagation of broadband acoustic signals in oceanic waveguides (review of publications of russian scientists). volume 621, pages 3-12. AIP, 2002.

[48] Michael B. Porter. The kraken normal mode program http://oalib.hlsresearch.com/modes/ acousticstoolbox/manual_html/kraken.html. 1997.

[49] Jorge E. Quijano, Lisa M. Zurk, and Daniel Rouseff. Demonstration of the invariance principle for active sonar. The Journal of the Acoustical Society of America, 123(3):1329-1337, 2008.

[50] Subramaniam D. Rajan, James F. Lynch, and George V. Frisk. Perturbative inversion methods for obtaining bottom geoacoustic parameters in shallow water. The Journal of the Acoustical Society of America, 82(3):998-1017, 1987.

[51] D. Rouseff and R. C. Spindel. Modeling the waveguide invariant as a distribution. In W. A. Kuperman and G. L. D'Spain, editors, Ocean Acoustic Interference Phenomena and Signal Processing, volume 621 of AIP Conf. Proc., pages 137-150, June 2002.

[52] Daniel Rouseff. Effect of shallow water internal waves on ocean acoustic striation pattern. Waves in Random and Complex Media, 11:377--393, 2001.

[53] Daniel Rouseff and Dajun Tang. Internal waves as a proposed mechanism for increasing ambient noise in an increasingly acidic ocean. The Journal of the Acoustical Society of America, 127(6):EL235-EL239, 2010.

[54] Hee Chun Song, W. A. Kuperman, and W. S. Hodgkiss. A time-reversal mirror with variable range focusing. The Journal of the Acoustical Society of America, 103(6):3234-3240, 1998. 
[55] Kathleen M. Stafford, Christopher G. Fox, and David S. Clark. Long-range acoustic detection and localization of blue whale calls in the northeast pacific ocean. The Journal of the Acoustical Society of America, 104(6):3616-3625, 1998.

[56] Hailiang Tao, G. Hickman, J.L. Krolik, and M. Kemp. Single hydrophone passive localization of transiting acoustic sources. IEEE OCEANS 2007 Europe, pages 1-3, June 2007.

[57] Hailiang Tao and Jeffrey L. Krolik. Waveguide invariant focusing for broadband beamforming in an oceanic waveguide. The Journal of the Acoustical Society of America, 123(3):1338-1346, 2008.

[58] Aaron M. Thode. Source ranging with minimal environmental information using a virtual receiver and waveguide invariant theory. The Journal of the Acoustical Society of America, 108(4):1582-1594, 2000.

[59] Aaron M. Thode, W. A. Kuperman, G. L. D’Spain, and W. S. Hodgkiss. Localization using bartlett matched-field processor sidelobes. The Journal of the Acoustical Society of America, 107(1):278-286, 2000.

[60] Ivan Tolstoy and C. S. Clay. Ocean Acoustics. American Institute of Physics, New York, 2nd edition, 1987.

[61] Harry L. Van Trees. Optimum Array Processing (Detection, Estimation, and Modulation Theory, Part IV). John Wiley and Sons Inc., New York, 2002.

[62] Altan Turgut, Marshall Orr, and Daniel Rouseff. Broadband source localization using horizontal-beam acoustic intensity striations. The Journal of the Acoustical Society of America, 127(1):73-83, 2010.

[63] Eric W. Weisstein. Cross-correlation theorem http://mathworld.wolfram.com/cross-correlationtheorem.html. Last viewed Nov. 10th, 2009.

[64] Eric W. Weisstein. Wiener-khinchin theorem http://mathworld.wolfram.com/wiener-khinchintheorem.html. Last viewed Nov. 10th, 2009. 
[65] Peter F. Worcester, Bruce D. Cornuelle, Matthew A. Dzieciuch, Walter H. Munk, Bruce M. Howe, James A. Mercer, Robert C. Spindel, John A. Colosi, Kurt Metzger, Theodore G. Birdsall, and Arthur B. Baggeroer. A test of basinscale acoustic thermometry using a large-aperture vertical array at $3250-\mathrm{km}$ range in the eastern north pacific ocean. The Journal of the Acoustical Society of America, 105(6):3185-3201, 1999.

[66] T. C. Yang. Beam intensity striations and applications. The Journal of the Acoustical Society of America, 113(3):1342-1352, 2003. 